\title{
ON GLOBAL FLUCTUATIONS FOR NON-COLLIDING PROCESSES $^{1}$
}

\author{
BY MAURICE DUITS
}

Royal Institute of Technology (KTH)

\begin{abstract}
We study the global fluctuations for a class of determinantal point processes coming from large systems of non-colliding processes and nonintersecting paths. Our main assumption is that the point processes are constructed by biorthogonal families that satisfy finite term recurrence relations. The central observation of the paper is that the fluctuations of multi-time or multi-layer linear statistics can be efficiently expressed in terms of the associated recurrence matrices. As a consequence, we prove that different models that share the same asymptotic behavior of the recurrence matrices, also share the same asymptotic behavior for the global fluctuations. An important special case is when the recurrence matrices have limits along the diagonals, in which case we prove Central Limit Theorems for the linear statistics. We then show that these results prove Gaussian Free Field fluctuations for the random surfaces associated to these systems. To illustrate the results, several examples will be discussed, including non-colliding processes for which the invariant measures are the classical orthogonal polynomial ensembles and random lozenge tilings of a hexagon.
\end{abstract}

1. Introduction. Random surfaces appearing in various models of integrable probability, such as random matrices and random tilings, are known to have a rich structure. A particular feature, one that has received much attention in recent years, is the Gaussian Free Field that is expected to appear as a universal field describing the global fluctuations of such random surfaces. Using the integrable structure, this has been rigorously verified in a number of models in the literature. For a partial list of references, see [6-9, 14, 19, 32, 37, 43]. The results so far indicate the universality of the Gaussian Free Field in this context is rather robust. In this paper, we will be interested in the global fluctuations for a particular class of models, namely that of non-colliding processes and ensembles of non-intersecting paths with determinantal correlations. For those models, we will provide a general principle that leads to Gaussian Free Field type fluctuations.

Non-colliding process and non-intersecting path ensembles form an important class of two-dimensional random interacting systems. For instance, Dyson showed [22] that the Gaussian Unitary Ensemble is the invariant measure for a system of

Received April 2016; revised March 2017.

${ }^{1}$ Supported by the Swedish Research Council (VR) Grant no. 2012-3128.

MSC2010 subject classifications. 60K35, 60F05, 42C05.

Key words and phrases. Non-colliding processes, Gaussian Free Field, Central Limit Theorems, determinantal point processes, orthogonal polynomials. 
non-colliding Ornstein-Uhlenbeck processes. Replacing the Ornstein-Uhlenbeck process by its radially squared version defines a similar stochastic dynamics for the Laguerre Unitary Ensemble [35]. In Section 3, we will recall a generalization to non-colliding processes for which the classical orthogonal polynomial ensembles (continuous and discrete) are the invariant measures. Another source of models that lead to non-intersecting paths are random tilings of planar domains. Lozenge tilings of a hexagon on a triangular lattice, for example, can alternatively be described by discrete random walks that start and end at opposite sites of the hexagon $[25,30]$. In that way, a probability measure on all tilings of the hexagon induces a non-intersecting path ensemble.

By the tandem of the Karlin-McGregor or Gessel-Lindström-Viennot theorem and the Eynard Mehta Theorem, it follows that many non-colliding process are determinantal point processes; see, for example, [31]. This makes them especially tractable for asymptotic analysis. A natural way to study the random surfaces appearing in this way is to analyze linear statistics for such determinantal point processes. The purpose of this paper is to prove Central Limit Theorems for multi-time or multi-layer linear statistics for a certain class of determinantal point processes. The novelty of the results lies in the fact that we do not use the correlation kernel as the starting point, but start from recurrences for the biorthogonal families that integrate the models, similar to the approach for one-dimensional biorthogonal ensembles in [13]. The conditions under which the results hold are easily verified in the classical ensembles. In particular, we will show that this Central Limit Theorem confirms the universality conjecture for the Gaussian Free Field in these models. We will illustrate our results by considering several examples, including dynamic extension of Unitary Ensembles and other ensembles related to (bi)orthogonal polynomials. In some of these classical examples, it should also be possible to prove the Central Limit Theorems in an alternative way using the asymptotics of the correlation kernel. However, in other examples (cf. Section 3.2) finding the asymptotics of the correlations kernel is known to be a serious challenge, but our conditions on the recurrences are verified with relative ease.

In the remaining part of the Introduction, we give an example of what type of results we will prove by discussing a classical model [22]. Consider the left picture shown in Figure 1 depicting $n$ Brownian bridges $t \mapsto \gamma_{j}(t)$ that start for $t=0$ at the origin and return there at $t=1$. We also condition the bridges never to collide. The $\gamma_{j}(t)$ turn out to have the same distribution as the ordered eigenvalues of an $n \times n$ Hermitian matrix for which the real and imaginary part of each entry independently (up to the symmetry) performs a Brownian bridge. Hence, at any given time $t \in(0,1)$ the locations $\gamma_{j}(t)$ have the exact same distribution of the appropriately scaled eigenvalues of a matrix taken randomly from the Gaussian Unitary Ensemble (=GUE). Note that in the original work [22] Dyson considered matrixdiffusions on Hermitian matrices driven by independent Ornstein-Uhlenbeck processes. The stationary case of his description is equivalent to the above after a 

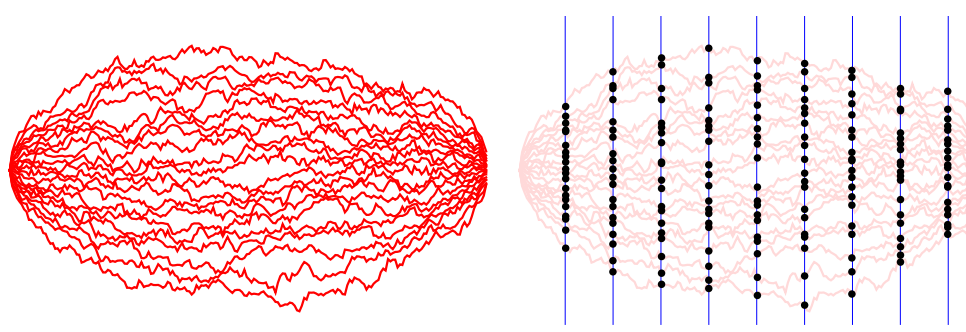

FIG. 1. The left picture shows a typical configuration of Brownian bridges that are conditioned never to collide. In the right picture, we take a number of vertical sections of the bridge.

simple change of variables; see, for example, [30] and will be discussed in Example 3.2 below. We also refer to $[1,2]$ for general references on Random Matrix Theory.

Because of the non-colliding condition, we can view the paths as level lines for a random surface. More precisely, if we define the height function by

$$
h_{n}(t, x)=\#\left\{j \mid \gamma_{j}(t) \leq x\right\},
$$

that is, $h_{n}(t, x)$ counts the number of paths directly below a given point $(t, x)$, then the trajectories are the lines where the stepped surface defined by the graph of $h_{n}(t, x)$ makes a jump by one. The question is what happens with $h_{n}$ when the size of the system grows large, that is, $n \rightarrow \infty$.

It turns out that as $n \rightarrow \infty$ the normalized height function $\frac{1}{n} h_{n}$ has an almost sure limit, also called the limit shape. Indeed, when $n \rightarrow \infty$ the paths will all accumulate on a region $\mathcal{E}$ that is usually referred to as the disordered region. We will assume that the original system is rescaled such that $\mathcal{E}$ does not depend on $n$ and is a nonempty open domain in $\mathbb{R}^{2}$. In fact, after a proper rescaling, the domain $\mathcal{E}$ is the ellipse

$$
\mathcal{E}=\left\{(t, x) \mid x^{2} \leq 4 t(1-t)\right\}
$$

It is well known that the eigenvalue distribution of a GUE matrix converges to the semi-circle law (see, e.g., [2]). This implies that we have the following limit for the height function

$$
\lim _{n \rightarrow \infty} \frac{1}{n} \mathbb{E} h_{n}(t, x)=\frac{1}{2 \pi t(1-t)} \int_{-\sqrt{4 t(1-t)}}^{x} \sqrt{4 t(1-t)-s^{2}} \mathrm{~d} s .
$$

The next question is about the fluctuations of the random surface, that is, the behavior of $h_{n}(t, x)-\mathbb{E} h_{n}(t, x)$. For a fixed point $(t, x)$, we note that $h_{n}(t, x)$ is a counting statistic counting the number of eigenvalues of a suitably normalized GUE matrix in a given semi-infinite interval $(-\infty, x]$. The variance for such a statistic is known to be growing logarithmically $\sim c \ln n$ as $n \rightarrow \infty$. Moreover, by dividing by the variance we find that

$$
\frac{h_{n}(t, x)-\mathbb{E} h_{n}(t, x)}{\sqrt{\operatorname{Var} h_{n}(t, x)}} \rightarrow N(0,1),
$$


as $n \rightarrow \infty$. The principle behind this result goes back to Costin-Lebowitz [15] and was later extended by Soshnikov [49]. However, if we consider the correlation between several points that are macroscopically far apart,

$$
\mathbb{E}\left[\prod_{j=1}^{R}\left(h_{n}\left(t_{j}, x_{j}\right)-\mathbb{E} h_{n}\left(t_{j}, x_{j}\right)\right)\right],
$$

we obtain a finite limit as $n \rightarrow \infty$. When $n \rightarrow \infty$, the random surface defined by the graph of $h_{n}(t, x)-\mathbb{E} h_{n}(t, x)$ converges to a rough surface. The pointwise limit does not make sense (due to the growing variance) but it has a limit as a generalized function. This generalized function is, up to coordinate transform, known in the literature as the Gaussian Free Field.

Since the Gaussian Free Field is a random generalized function, it is natural to pair it with a test function $\phi$, that is,

$$
\left\langle h_{n}, \phi\right\rangle=\iint h_{n}(t, x) \phi(t, x) \mathrm{d} t \mathrm{~d} x
$$

(as we will show in Section 2.4 the Dirichlet pairing is more appropriate, also including a coordinate transform, but this simpler pairing does show the essential idea). Then by writing $h_{n}(t, x)=\sum_{j=1}^{n} \chi_{\left(-\infty, \gamma_{j}(t)\right]}(x)$ and by a discretization of the time integral, we obtain

$$
\begin{aligned}
\left\langle h_{n}, \phi\right\rangle & =\sum_{j=1}^{n} \iint_{-\infty}^{\gamma_{j}(t)} \phi(t, x) \mathrm{d} x \mathrm{~d} t \\
& =\sum_{m=1}^{N} \sum_{j=1}^{n}\left(t_{m+1}-t_{m}\right) \int_{-\infty}^{\gamma_{j}\left(t_{m}\right)} \phi\left(t_{m}, x\right) \mathrm{d} x,
\end{aligned}
$$

where we choose $N \in \mathbb{N}$ time points $t_{m} \in(0,1)$ such that

$$
0=t_{0}<t_{1}<\cdots<t_{N}<t_{N+1}=1,
$$

and typically want the mesh $\sup _{m=0, \ldots, N}\left(t_{m+1}-t_{m}\right)$ to be small. The fact of the matter is that the right-hand side is a linear statistic for the point process on $\{1, \ldots, N\} \times \mathbb{R}$ defined by the locations

$$
\left\{\left(m, \gamma_{j}\left(t_{m}\right)\right)\right\}_{j=1, m=1}^{n, N} \text {. }
$$

In other words, the pairing in (1.3) naturally leads us to studying linear statistics $X_{n}(f)$ defined by

$$
X_{n}(f)=\sum_{m=1}^{N} \sum_{j=1}^{n} f\left(m, \gamma_{j}\left(t_{m}\right)\right),
$$

for a function $f$ on $\{1, \ldots, N\} \times \mathbb{R}$. The central question of the paper is to ask for the limiting behavior, as $n \rightarrow \infty$, of the fluctuations of $X_{n}(f)-\mathbb{E} X_{n}(f)$ for sufficiently smooth functions $f$. A particular consequence of the main results is the following. 
Proposition 1.1. Let $f:\{1, \ldots, N\} \times \mathbb{R} \rightarrow \mathbb{R}$ such that $x \mapsto f(m, x)$ is continuously differentiable and grows at most polynomially for $x \rightarrow \pm \infty$. Then the linear statistic (1.7) for the point process (1.6) with $\left\{t_{m}\right\}_{m=1}^{N}$ as in (1.5) satisfies

$$
X_{n}(f)-\mathbb{E} X_{n}(f) \rightarrow N\left(0, \sigma_{f}^{2}\right)
$$

as $n \rightarrow \infty$, where

$$
\sigma(f)^{2}=\sum_{m_{1}, m_{2}=1}^{N} \sum_{k=1}^{\infty} \mathrm{e}^{-\left|\tau_{m_{1}}-\tau_{m_{2}}\right| k} k f_{k}^{\left(m_{1}\right)} f_{k}^{\left(m_{2}\right)},
$$

with $\tau_{m}=\frac{1}{2} \ln t_{m} /\left(1-t_{m}\right)$ and

$$
f_{k}^{(m)}=\frac{1}{\pi} \int_{0}^{\pi} f\left(m, 2 \sqrt{t_{m}\left(1-t_{m}\right)}-\cos \theta\right) \cos k \theta \mathrm{d} \theta,
$$

for $k \in \mathbb{N}$.

This Central Limit Theorem is a special case of a more general theorem that we will state in the next section. The main point of the present paper is to show that such results follow from a general principle for models that have a determinantal structure for which the integrating functions (i.e., the Hermite polynomials in the above example) satisfy a finite term recurrence. The proof of Proposition 1.1 will be discussed in Example 3.2 (see also [6] for a similar statement in the context of stochastic evolutions for Wigner matrices). The precise connection to the Gaussian Free Field will be explained in Section 2.4.

Overview of the rest of the paper. In Section 2, we will formulate the model that we will consider and state our main results, including various corollaries. The proofs of those corollaries will also be given in Section 2, but the proofs of the main results, Theorems 2.2, 2.5, 2.6 and 2.9 will be given in Section 6. Our approach is a connection to recurrence matrices, which will be explained in Section 4 . Then in Section 5, we will analyze the asymptotic behavior of a general Fredholm determinant from which the proofs of the main results are special cases. Finally, in Section 3 we will provide ample examples to illustrate our results.

2. Statement of results. In this section, we will discuss the general model that we will consider and state our main results. Some proofs are postponed to later sections.

2.1. The model. Determinantal point processes that come from non-colliding process and non-intersecting paths have a particular form. In this paper, we will 
therefore consider probability measures on points $\left\{x_{j, m}\right\}_{j, m=1}^{n, N}$ that can be written as the product of several determinants

$$
\begin{gathered}
\frac{1}{Z_{n}} \operatorname{det}\left(\phi_{j, 1}\left(x_{1, k}\right)\right)_{j, k=1}^{n} \prod_{m=1}^{N-1} \operatorname{det}\left(T_{m}\left(x_{m, i}, x_{m+1, j}\right)\right)_{i, j=1}^{n} \\
\quad \times \operatorname{det}\left(\psi_{j, N}\left(x_{N, k}\right)\right)_{j, k=1}^{n} \prod_{m=1}^{N} \prod_{j=1}^{n} \mathrm{~d} \mu_{m}\left(x_{m, j}\right),
\end{gathered}
$$

where $Z_{n}$ is a normalizing constant, $\mathrm{d} \mu_{m}$ are Borel measures on $\mathbb{R}, \phi_{j, 1} \in \mathbb{L}_{2}\left(\mu_{N}\right)$ and $\psi_{j, N} \in \mathbb{L}_{2}\left(\mu_{1}\right)$. The function $T_{m}$ is such that the integral operator $\mathcal{T}_{m}$ : $\mathbb{L}_{2}\left(\mu_{m}\right) \rightarrow \mathbb{L}_{2}\left(\mu_{m+1}\right)$ defined by

$$
\mathcal{T}_{m} f(y)=\int f(x) T_{m}(x, y) \mathrm{d} \mu_{m}(x),
$$

is a bounded operator. We think of $\left\{x_{j, m}\right\}_{j, m=1}^{n, N}$ as the positions of $n$ non-colliding paths (indexed by $j$ ) at $N$ different points in time (indexed by $m$ ).

The form of (2.1) may look very special at first, but it appears often when dealing with non-colliding processes and non-intersecting paths; see, example, [31] and the references therein. The key is the Karlin McGregor Theorem in the continuous setting or the Gessel-Lindström-Viennot theorem in the discrete setting that say that the transition probabilities of non-colliding processes can be expressed as determinants of a matrix constructed out of the transition probability for a single particle. We will discuss several explicit examples in Section 3.

It is standard that without loss of generality we can assume that

$$
\int \psi_{j, N}(x) \mathcal{T}_{N-1} \mathcal{T}_{N-2} \cdots \mathcal{T}_{1} \phi_{k, 1}(x) \mathrm{d} \mu_{N}(x)=\delta_{j k}
$$

for $j, k=1, \ldots, n$. To show this, we first recall Andreiéf's identity: For any measure $v$ and $f_{j}, g_{j} \in \mathbb{L}_{2}(v)$ for $j=1, \ldots, n$, we have

$$
\begin{gathered}
\int \cdots \int \operatorname{det}\left(f_{j}\left(x_{k}\right)\right)_{j, k=1}^{n} \operatorname{det}\left(g_{j}\left(x_{k}\right)\right)_{j, k=1}^{n} \mathrm{~d} v\left(x_{1}\right) \cdots \mathrm{d} v\left(x_{n}\right) \\
=n ! \operatorname{det}\left(\int f_{j}(x) g_{k}(x) \mathrm{d} v(x)\right)_{j, k=1}^{n} \cdot
\end{gathered}
$$

Then, by iterating (2.3), we see that $Z_{n}$ can be expressed as the determinant of the Gram-matrix associated to $\phi_{i, 1}$ and $\psi_{j, N}$, that is,

$$
Z_{n}=(n !)^{N} \operatorname{det}\left(\int \psi_{i, N}(x) \mathcal{T}_{N-1} \mathcal{T}_{N-2} \cdots \mathcal{T}_{1} \phi_{j, 1}(x) \mathrm{d} \mu_{N}(x)\right)_{i, j=1}^{n} .
$$

Since $Z_{n}$ cannot vanish, it means that Gram-matrix is nonsingular. The fact of the matter is that by linearity of the determinant, we can replace the $\phi_{j, 1}$ 's and $\psi_{k, N}$ 's in the determinants in (2.1) by any other linear combinations of those functions, 
as long as the resulting family is linearly independent. A particular choice, for example, by using the singular value decomposition of the original Gram-matrix, is to make sure that the new Gram-matrix becomes the identity. In other words, without loss of generality we can assume that we $\phi_{j, 1}$ and $\psi_{k, N}$ are such that (2.2) holds. This also shows that in that case $Z_{n}=(n !)^{N}$.

An important role in the analysis is played by the functions

$$
\phi_{j, m}=\mathcal{T}_{m-1} \cdots \mathcal{T}_{1} \phi_{j, 1}, \quad \psi_{j, m}=\mathcal{T}_{m}^{*} \cdots \mathcal{T}_{N-1}^{*} \psi_{j, N},
$$

for $m=1, \ldots, N$, where $\mathcal{T}_{m}^{*}$ stands for the adjoint of $\mathcal{T}_{m}$ which is given by

$$
\mathcal{T}_{m}^{*} f(x)=\int f(y) T_{m}(x, y) \mathrm{d} \mu_{m+1}(y) .
$$

Note that it follows from (2.2) that

$$
\int \phi_{j, m}(x) \psi_{k, m}(x) \mathrm{d} \mu_{m}(x)=\delta_{j k},
$$

for $j, k=1, \ldots, n$ and $m=1, \ldots, N$. The marginals in (2.1) for the points $\left\{\left(m, x_{j, m}\right)\right\}_{j=1}^{n}$ for a fixed $m$ are given by the measure

$$
\frac{1}{n !} \operatorname{det}\left(\phi_{j, m}\left(x_{m, k}\right)\right)_{j, k=1}^{n} \operatorname{det}\left(\psi_{j, m}\left(x_{m, k}\right)\right)_{j, k=1}^{n} \mathrm{~d} \mu_{m}\left(x_{1, m}\right) \cdots \mathrm{d} \mu_{m}\left(x_{n, m}\right) .
$$

A probability measure of this type is known in the literature as a biorthogonal ensemble [4].

It is well known that, by the Eynard Mehta Theorem, measures of the form (2.1) are examples of determinantal point processes. We recall that a determinantal point process is a point process for which the $k$-point correlation functions can be written as $k \times k$ determinants constructed out of a single function of two variables, called the correlation kernel. More precisely, there exists a $K_{n, N}$ such that for any test function $g$ we have

$$
\begin{aligned}
& \mathbb{E}\left[\prod_{m=1, j=1}^{N, n}\left(1+g\left(m, x_{m, j}\right)\right)\right] \\
& \quad=\sum_{\ell=0}^{\infty} \int_{\Lambda} g\left(\eta_{1}\right) \cdots g\left(\eta_{\ell}\right) \operatorname{det}\left(K_{n, N}\left(\eta_{i}, \eta_{j}\right)\right)_{i, j=1}^{\ell} \mathrm{d} v\left(\eta_{1}\right) \cdots \mathrm{d} v\left(\eta_{\ell}\right),
\end{aligned}
$$

where $\Lambda=\{1,2, \ldots, N\} \times \mathbb{R}$ and $v$ is a measure on $\Lambda$, called the reference measure. For the point process defined by (2.1), this kernel has the form

$$
\begin{aligned}
K_{n, N}\left(m_{1}, x_{1}, m_{2}, x_{2}\right) & \\
= & \begin{cases}\sum_{k=1}^{n} \phi_{j, m_{1}}\left(x_{1}\right) \psi_{j, m_{2}}\left(x_{2}\right), & \text { if } m_{1} \geq m_{2}, \\
\sum_{k=1}^{n} \phi_{j, m_{1}}\left(x_{1}\right) \psi_{j, m_{2}}\left(x_{2}\right)-T_{m_{1}, m_{2}}\left(x_{1}, x_{2}\right), & \text { if } m_{1}<m_{2},\end{cases}
\end{aligned}
$$


with reference measure $v=\sum_{m=1}^{N} \delta_{m} \times \mu_{m}$. Here, $T_{m_{1}, m_{2}}\left(x_{1}, x_{2}\right)$ stands for the integral kernel for the integral operator $\mathcal{T}_{m_{1}} \mathcal{T}_{m_{1}+1} \cdots \mathcal{T}_{m_{2}-1}$. For more details and background on determinantal point processes, we refer to [5, 31, 39, 48].

For a determinantal point process, all information is in one way or the other encoded in the correlation kernel. For that reason, a common approach to various results for determinantal point processes goes by an analysis of the kernel and its properties. However, in this paper we use an alternative approach for analyzing the global fluctuations. We follow the idea of [13] and assume that the biorthogonal families admit a recurrence.

ASSUMPTION 2.1. We assume that $\left\{\phi_{j, 1}\right\}_{j=1}^{N}$ and $\left\{\psi_{j, N}\right\}_{j=1}^{N}$ can be extended to families $\left\{\phi_{j, 1}\right\}_{j=1}^{\infty}$ and $\left\{\psi_{j, N}\right\}_{j=1}^{\infty}$ such that the functions defined by

$$
\phi_{j, m}=\mathcal{T}_{m-1} \cdots \mathcal{T}_{1} \phi_{1, m}, \quad \psi_{j, m}=\mathcal{T}_{m}^{*} \cdots \mathcal{T}_{N-1}^{*} \psi_{j, N},
$$

for $m=1, \ldots, N$, have the properties

1. Biorthogonality:

$$
\int \psi_{j, N}(x) \mathcal{T}_{N-1} \cdots \mathcal{T}_{1} \phi_{k, 1}(x) \mathrm{d} \mu_{N}(x)=\delta_{j k},
$$

for $j, k=1,2, \ldots$.

2. Recursion: for each $m \in\{1, \ldots, N\}$ there is a banded matrix $\mathbb{J}_{m}$ such that

$$
x\left(\begin{array}{c}
\phi_{0, m}(x) \\
\phi_{1, m}(x) \\
\phi_{2, m}(x) \\
\vdots
\end{array}\right)=\mathbb{J}_{m}\left(\begin{array}{c}
\phi_{0, m}(x) \\
\phi_{1, m}(x) \\
\phi_{2, m}(x) \\
\vdots
\end{array}\right) .
$$

We will denote the bandwidth by $\rho$, that is,

$$
\left(\mathbb{J}_{m}\right)_{k, l}=0 \quad \text { if }|k-l| \geq \rho .
$$

We will assume that $\rho$ does not depend on $m$ or $n$ (but $\mathbb{J}_{m}$ may also depend on $n$ ).

Note that (2.9) and the banded structure of $\mathbb{J}_{m}$ means that the functions $\phi_{j, m}$ satisfy finite term recurrence relation

$$
x \phi_{k, m}(x)=\sum_{|j| \leq \rho}\left(\mathbb{J}_{m}\right)_{k, k+j} \phi_{k+j, m}(x) .
$$

The number of terms in the recurrence equals the number of nontrivial diagonals, which is at most $2 \rho+1$. Also note that by biorthogonality we have

$$
\left(\mathbb{J}_{m}\right)_{k l}=\int x \phi_{k, m}(x) \psi_{l, m}(x) \mathrm{d} \mu_{m}(x) .
$$

Finally, we mention that although the arguments in this paper can likely by adjusted to allow for a varying bandwidth (but keeping the bandwidth uniformly 
bounded in $m, n, N)$, in the relevant examples we always have a fixed bandwidth independent of $n, m$.

An important special class of examples that we will study in this paper is when the biorthogonal families are related to orthogonal polynomials. If each $\mu_{m}$ has finite moments, then we can define $p_{j, m}$ as the polynomial of degree $j$ with positive leading coefficient such that

$$
\int p_{j, m}(x) p_{k, m}(x) \mathrm{d} \mu_{m}(x)=\delta_{j k} .
$$

As we will see in Section 3, in many examples we end up with a measure (2.1) with $\phi_{j, 1}=c_{j, 1} p_{j-1,1}, \psi_{j, N}=p_{j-1, N} / c_{j, N}$ and

$$
T_{m}(x, y)=\sum_{j=1}^{\infty} \frac{c_{j, m+1}}{c_{j, m}} p_{j-1, m}(x) p_{j-1, m+1}(y),
$$

for some coefficients $c_{j, m} \neq 0$. In that case, we find

$$
\phi_{j, m}(x)=c_{j, m} p_{j-1, m} \quad \text { and } \quad \psi_{j, m}(x)=\frac{1}{c_{j, m}} p_{j-1, m}(x) .
$$

Such examples satisfy Assumption 2.1. Indeed, it is classical that the orthogonal polynomials satisfy a three-term recurrence

$$
x p_{k, m}(x)=a_{k+1, m} p_{k+1, m}(x)+b_{k, m} p_{k, m}(x)+a_{k, m} p_{k-1, m}(x),
$$

for coefficients $a_{k, m}>0$ and $b_{k, m} \in \mathbb{R}$. This recurrence can be written as

$$
x\left(\begin{array}{c}
p_{0, m}(x) \\
p_{1, m}(x) \\
p_{2, m}(x) \\
\vdots
\end{array}\right)=\mathcal{J}_{m}\left(\begin{array}{c}
p_{0, m}(x) \\
p_{1, m}(x) \\
p_{2, m}(x) \\
\vdots
\end{array}\right)
$$

The matrix $\mathcal{J}_{m}$ is then a symmetric tridiagonal matrix containing the recurrence coefficients, also called the Jacobi matrix/operator associated to $\mu_{m}$. It is not hard to check that in this situation, Assumption 2.1 is satisfied with

$$
\left(\mathbb{J}_{m}\right)_{k l}=\frac{c_{k, m}}{c_{l, m}}\left(\mathcal{J}_{m}\right)_{k l} \text {. }
$$

We stress that such a recurrence relation is not special for orthogonal polynomials only, but appear often in the presence of an orthogonality condition. For instance, multiple orthogonal polynomial ensembles [38] also appear in the context of non-colliding processes, such as external source models. Multiple orthogonal polynomials satisfy recurrence relations involving more terms than only three; see, for example, [50].

Finally, note that in the example in the Introduction, it was needed to rescale the process as $n \rightarrow \infty$. This rescaling means that all the parameters will depend on $n$. Therefore, we will allow $\mu_{m}, \phi_{j, m}$ and $\psi_{j, m}$ to depend on $n$, but for clarity reasons we will suppress this dependence in the notation. 
2.2. Fluctuations of linear statistics for fixed $N$. We will study linear statistics for the determinantal point process. That is, for a function $f:\{0,1, \ldots, N\} \times \mathbb{R} \rightarrow$ $\mathbb{R}$ we define

$$
X_{n}(f)=\sum_{m=0}^{N} \sum_{j=1}^{n} f\left(m, x_{j, m}\right),
$$

where $\left\{\left(m, x_{j, m}\right)\right\}_{j=1, m=1}^{n, N}$ are sampled from a probability measure of the form (2.1) satisfying Assumption 2.1. As we will see, the linear statistics $X_{n}(f)$ admit a useful representation in terms of the recurrence matrices $\mathbb{J}_{m}$. In fact, one of the main points of the paper is that for studying linear statistics, this representation appears to be more convenient than the representation in terms of the correlation kernel $K_{n, N}$. In many interesting examples, the asymptotic study of the relevant parts of $\mathbb{J}_{m}$ are trivial, whereas the asymptotic analysis (in all the relevant regimes) of the kernel is usually tedious.

The central observation of this paper is that the fluctuation of the linear statistic depend strongly on just small part of the operators $\mathbb{J}_{m}$, More precisely, the coefficients $\left(\mathbb{J}_{m}\right)_{n+k, n+l}$ for fixed $k, l$ are dominant in the fluctuations for large $n$. The other coefficients only play a minor role. Two different models for which these coefficients behave similarly have the same fluctuations. This is the content of the first main result.

THEOREM 2.2. Consider two probability measures of the form (2.1) satisfying Assumption 2.1 and denote expectations with $\mathbb{E}$ and $\tilde{\mathbb{E}}$ and the banded matrices by $\mathbb{J}_{m}$ and $\tilde{\mathbb{J}}_{m}$. Assume that for any $k, l \in \mathbb{Z}$ the sequence $\left\{\left(\tilde{\mathbb{J}}_{m}\right)_{n+k, n+l}\right\}_{n}$ is bounded and

$$
\lim _{n \rightarrow \infty}\left(\left(\mathbb{J}_{m}\right)_{n+k, n+l}-\left(\tilde{\mathbb{J}}_{m}\right)_{n+k, n+l}\right)=0 .
$$

Then for any function $f:\{0,1, \ldots, N\} \times \mathbb{R} \rightarrow \mathbb{R}$ such that $f(m, x)$ is a polynomial in $x$, we have for $k \in \mathbb{N}$,

$$
\mathbb{E}\left[\left(X_{n}(f)-\mathbb{E} X_{n}(f)\right)^{k}\right]-\widetilde{\mathbb{E}}\left[\left(X_{n}(f)-\widetilde{\mathbb{E}} X_{n}(f)\right)^{k}\right] \rightarrow 0,
$$

as $n \rightarrow \infty$.

The proof of this theorem will be given in Section 6 .

This result is a genuine universality result, in the sense that there is no specification of a limit. If two families of models have the same asymptotic behavior of the recurrence matrices, then the fluctuations are also the same. As a consequence, after computing the limiting behavior for a particular example, we obtain the same result for all comparable processes.

The natural question is then what the typical behaviors are that one observes in the models of interest. As we will illustrate in Section 2.4, one important example is when the recurrence coefficients have a limiting value or, more precisely, 
the matrices $\mathbb{J}_{m}$ have limits along the diagonals. The fluctuations in that case are described by the following theorem.

THEOREM 2.3. Consider a probability measure of the form (2.1) satisfying Assumption 2.1. Assume that there exists $a_{j}^{(m)} \in \mathbb{R}$ such that

$$
\lim _{n \rightarrow \infty}\left(\mathbb{J}_{m}\right)_{n+k, n+l}=a_{k-l}^{(m)},
$$

for $k, l \in \mathbb{Z}$ and $m=1, \ldots, N$. Then for any function $f:\{1, \ldots, N\} \times \mathbb{R} \rightarrow \mathbb{R}$ such that $f(m, x)$ is a polynomial in $x$, we have

$$
X_{n}(f)-\mathbb{E} X_{n}(f)
$$

$$
\rightarrow N\left(0,2 \sum_{m_{1}=1}^{N} \sum_{m_{2}=m_{1}+1}^{N} \sum_{k=1}^{\infty} k f_{k}^{\left(m_{1}\right)} f_{-k}^{\left(m_{2}\right)}+\sum_{m=1}^{N} \sum_{k=1}^{\infty} k f_{k}^{(m)} f_{-k}^{(m)}\right)
$$

where

$$
f_{k}^{(m)}=\frac{1}{2 \pi \mathrm{i}} \oint_{|z|=1} f\left(m, a^{(m)}(z)\right) \frac{\mathrm{d} z}{z^{k+1}}
$$

and $a^{(m)}(z)=\sum_{j} a_{j}^{(m)} z^{j}$

REMARK 2.1. Note that each $\mathbb{J}_{m}$ is banded and hence only finitely many $a_{j}^{(m)}$ are nonzero. In particular, each $a^{(m)}(z)$ is a Laurent polynomial.

The proof of this theorem will be given in Section 6 .

The latter result in particular applies when we are in the situation of orthogonal polynomials $(2.10)$. In that case, the following corollary will be particularly useful to us.

COROLLARY 2.4. Consider a probability measure of the form (2.1) with $\phi_{j, m}$ and $\psi_{j, m}$ as in (2.10). Assume that for $k, \ell \in \mathbb{Z}$ with $|k-\ell| \leq 1$ we have

$$
\lim _{n \rightarrow \infty}\left(\mathcal{J}_{m}\right)_{n+k, n+l}=a_{|k-l|}^{(m)}
$$

for some $a_{0}^{(m)} \in \mathbb{R}$ and $a_{1}^{(m)}>0$ and

$$
\lim _{n \rightarrow \infty} \frac{c_{n+k, m}}{c_{n+\ell, m}}=\mathrm{e}^{\tau_{m}(\ell-k)},
$$

for some $\tau_{1}<\tau_{2}<\cdots<\tau_{m}$. Then for any function $f:\{1, \ldots, N\} \times \mathbb{R} \rightarrow \mathbb{R}$ such 
that $f(m, x)$ is a polynomial in $x$ we have

$$
\begin{aligned}
X_{n}(f) & -\mathbb{E} X_{n}(f) \\
& \rightarrow N\left(0, \sum_{m_{1}=1}^{N} \sum_{m_{2}=1}^{N} \sum_{k=1}^{\infty} k \mathrm{e}^{-\left|\tau_{m_{1}}-\tau_{m_{2}}\right| k} \hat{f}_{k}^{\left(m_{1}\right)} \hat{f}_{k}^{\left(m_{2}\right)}\right),
\end{aligned}
$$

where

$$
\hat{f}_{k}^{(m)}=\frac{1}{\pi} \int_{0}^{\pi} f\left(m, a_{0}^{(m)}+2 a_{1}^{(m)} \cos \theta\right) \cos k \theta \mathrm{d} \theta .
$$

PROOF. This directly follows from Theorem 2.3, the relation (2.12) and a rewriting of the limiting variance. For the latter, note that by a rescaling and a symmetry argument (2.17) can be written as

$$
f_{k}^{(m)}=\mathrm{e}^{-\tau_{m} k} \hat{f}_{k}^{(m)}
$$

Moreover, by $\hat{f}_{k}^{(m)}=\hat{f}_{-k}^{(m)}$ and the fact that $\tau_{\ell}<\tau_{k}$ for $\ell<k$, we can symmetrize the limiting variance in Theorem 2.3 to obtain the one in (2.20).

As we will see in Section 3, Theorem 2.3 and Corollary 2.4 cover several interesting examples. Many of the examples involve classical orthogonal polynomial ensembles for which the conditions are straightforward to check. In those classical examples, it should also be possible to give alternative proofs based on asymptotics for double integral formulas for the correlation kernels. However, we also discuss examples (cf. Section 3.2) where there structure of the correlation kernel is more complicated and no useful double integral representation exists. However, it is still possible to check the above conditions with relative ease. The reason for this is that finding asymptotics of the recurrence coefficients as needed in the theorem is an essentially simpler problem than finding asymptotics for the correlation kernel.

In case $N=1$, the determinantal point process reduces to the definition of a biorthogonal ensemble [5]. In this situation, the above results are already proved by the author and Breuer [13]. In that paper, the approach using recurrence matrices was used for the first time and later used again in a mesoscopic analysis for orthogonal polynomials ensembles [12]. The results in [13] are a generalization of various earlier works in the determinantal setting and there is a vast amount of references on the subject. We only single out the influential work of Johansson [28] on Unitary Ensembles (and extensions to general $\beta$ ) and refer to [13] for further references. However, much less is known in the case of $N>1$. To the best of the author's knowledge, the statement above is the first general result for multi-time or multi-layer linear statistics for determinantal point processes. 
REMARK 2.2. The conditions in both Theorem 2.2 and 2.3 can be relaxed. In fact, we only need the limits (2.13) along a subsequence $\left\{n_{k}\right\}_{k}$ to conclude (2.14) along that same subsequence. Similarly, for the limits in Theorem 2.3 and Corollary 2.4. For the case $N=1$ and $\mathcal{J}$, the Jacobi operator associated with the orthogonal polynomials corresponding to the measure $\mu$, this relates the study of possible limit theorems for the linear statistic to the study of right limits of the Jacobi operator. For the interested reader, we refer to the discussion in [13]. However, in the present setup this generality seems less relevant.

REMARK 2.3. The conditions in both Theorem 2.2 and 2.3 are not sufficient to guarantee that a limit shape exists, that is, we do not know (nor need) the limit of $\frac{1}{n} \mathbb{E} X_{n}(f)$.

REMARK 2.4. In Corollary 2.4, it is easy to see that the variance is positive. In fact, in that case the variance can also be written in a different form that will be useful to us. We recall the standard integral

$$
\mathrm{e}^{-k|\tau|}=\frac{1}{\pi} \int_{-\infty}^{\infty} \frac{k}{k^{2}+\omega^{2}} \mathrm{e}^{-\mathrm{i} \omega \tau} \mathrm{d} \tau .
$$

By inserting this back into (2.20) and a simple reorganization, we see that the limiting variance can be written as

$$
\frac{1}{\pi} \sum_{k=1}^{\infty} \int_{-\infty}^{\infty}\left|\sum_{m=1}^{N} \mathrm{e}^{-\mathrm{i} \tau_{m} \omega} k f_{k}^{(m)}\right|^{2} \frac{\mathrm{d} \omega}{k^{2}+\omega^{2}} .
$$

This will be of use later on when we explain the connection of the above results with the Gaussian Free Field.

In the general case, the limiting variance is of course also positive, but this is not evident from the expression due to the lack of symmetry. This feature is already present in the $N=1$ case, as discussed in [13].

In the situation of Corollary 2.4, we can formulate natural conditions that allow us to extend Theorem 2.3 so that it holds for more general functions $f$. In that case, we will prove that the variance is continuous with respect to the $C^{1}$ norm. Hence we can try to extend the theorem to $C^{1}$ functions by polynomial approximation. For such an approximation, it is convenient to work on a compact set.

THEOREM 2.5. Suppose all the conditions in Corollary 2.4 hold and in addition there exists a compact set $E \subset \mathbb{R}$ such that either

(1) all supports $S\left(\mu_{m}^{(n)}\right) \subset E$ for $n \in \mathbb{N}$ and $m=1, \ldots, N$, or, more generally, 
(2) for every $k \in \mathbb{N}$ and $m=1, \ldots, N$, we have

$$
\int_{\mathbb{R} \backslash E}|x|^{k} K_{n, N}(m, x, m, x) \mathrm{d} \mu_{m}(x)=o(1 / n),
$$

as $n \rightarrow \infty$.

Then the conclusion of Corollary 2.4 also holds for any $f:\{1, \ldots, N\} \times \mathbb{R} \rightarrow \mathbb{R}$ such that for $m \in\{1, \ldots, N\}$ the map $x \mapsto f(m, x)$ is a $C^{1}$ function that grows at most polynomially at $\pm \infty$.

The proof of this theorem will be given in Section 6 .

The conditions in the theorem are rather mild. In case of unbounded supports, one can often show in the classical situations, by standard asymptotic methods such as classical steepest descent arguments or Riemann-Hilbert techniques, that the second condition is satisfied with exponentially small terms at the right-hand side, instead of only $o(1 / n)$.

2.3. Varying $N_{n}$. Motivated by the example of non-colliding Brownian bridges in the Introduction, the natural question rises whether we can allow $N_{n}$ to depend on $n$ and such that $N_{n} \rightarrow \infty$. Indeed, in that example we wanted to view the discrete sum (1.4) as a Riemann sum. Hence we will now consider probability measures of the form (2.1) with $N=N_{n}$ and keep in mind that in many examples we have $T_{m}=P_{t_{m+1}-t_{m}}$ for some transition probability function $P_{t}$ and sampling times $t_{m}$.

We start with a partitioning

$$
\alpha=t_{0}^{(n)}<t_{1}^{(n)}<t_{2}^{(n)}<\cdots<t_{N}^{(n)}<t_{N+1}^{(n)}=\beta,
$$

of an interval $I=[\alpha, \beta] \subset \mathbb{R}$ such that

$$
\sup _{m}\left(t_{m+1}^{(n)}-t_{m}^{(n)}\right) \rightarrow 0
$$

as $n \rightarrow \infty$. And then, for a function on $g: I \times \mathbb{R} \rightarrow \mathbb{R}$, we ask for the equivalent statement of Theorems 2.2, 2.3 and 2.5 for the linear statistic

$$
Y_{n}(g)=\sum_{m=1}^{N_{n}} \frac{1}{t_{m+1}^{(n)}-t_{m}^{(n)}} \sum_{j=1}^{n} g\left(t_{m}^{(n)}, x_{j, m}\right) .
$$

The first result is that Theorem 2.2 continues to hold when the limits (2.13) hold uniformly in $m$.

THEOREM 2.6. Let $\left\{N_{n}\right\}_{n}$ be a sequence of integers such that $N_{n} \rightarrow \infty$ as $n \rightarrow \infty$. Consider two probability measures of the form (2.1) with $N=N_{n}$ and satisfying Assumption 2.1 and denote the banded matrices by $\mathbb{J}_{m}$ and $\tilde{\mathbb{J}}_{m}$ for $m=$ $1, \ldots, N_{n}$. 
Assume that for any $k, l \in \mathbb{Z}$ the set $\left\{\left(\tilde{\mathbb{J}}_{m}\right)_{n+k, n+l}\right\}_{m=1, n=1}^{N_{n}, \infty}$ is bounded and

$$
\lim _{n \rightarrow \infty} \sup _{m=1, \ldots, N_{n}}\left|\left(\mathbb{J}_{m}\right)_{n+k, n+l}-\left(\tilde{\mathbb{J}}_{m}\right)_{n+k, n+l}\right|=0 .
$$

Then for any function $g$ such that $g(t, x)$ is a polynomial in $x$ we have, for $k \in \mathbb{N}$ and $Y_{n}(g)$ as in (2.24),

$$
\mathbb{E}\left[\left(Y_{n}(g)-\mathbb{E} Y_{n}(g)\right)^{k}\right]-\widetilde{\mathbb{E}}\left[\left(Y_{n}(g)-\widetilde{\mathbb{E}} Y_{n}(g)\right)^{k}\right] \rightarrow 0,
$$

as $n \rightarrow \infty$.

Also Theorem 2.3 has an extension to the varying $N_{n}$ setting.

THEOREM 2.7. Let $\left\{N_{n}\right\}_{n}$ be a sequence of integers such that $N_{n} \rightarrow \infty$ as $n \rightarrow \infty$ and suppose that for each $n$ we have a probability measure of the form (2.1) with $N=N_{n}$ and satisfying Assumption 2.1.

Assume that there exist piecewise continuous functions $a_{k}(t)$ on the interval I such that, for $k, l \in \mathbb{Z}$,

$$
\lim _{n \rightarrow \infty} \sup _{m=1, \ldots, N_{n}}\left|\left(\mathbb{J}_{m}\right)_{n+k, n+l}-a_{k-l}\left(t_{m}^{(n)}\right)\right|=0 .
$$

Then for any function $g: I \times \mathbb{R} \rightarrow \mathbb{R}$ such that $g(t, x)$ is piecewise continuous in $t$ and a polynomial in $x$, we have that $Y_{n}(g)$ as defined in (2.24) satisfies

$$
Y_{n}(g)-\mathbb{E} Y_{n}(g) \rightarrow N\left(0, \sum_{k=1}^{\infty} 2 \iint_{\alpha<t_{1}<t_{2}<\beta} k g_{k}\left(t_{1}\right) g_{-k}\left(t_{2}\right) \mathrm{d} t_{1} \mathrm{~d} t_{2}\right)
$$

as $n \rightarrow \infty$, with

$$
g_{k}(t)=\frac{1}{2 \pi \mathrm{i}} \oint_{|z|=1} g\left(t, \sum_{\ell} a_{\ell}(t) z^{\ell}\right) \frac{\mathrm{d} z}{z^{k+1}} .
$$

As before, in the special case that we deal with orthogonal polynomials the latter theorem takes the following form.

COROLlary 2.8. Let $a_{0}(t), a_{1}(t)$ and $\tau(t)$ be piecewise continuous functions on an interval $I$ and assume that $\tau(t)$ is increasing.

Suppose that for each $n$ we have a probability measure of the form (2.1) satisfying Assumption 2.1 with $\phi_{j, m}$ and $\psi_{j, m}$ as in (2.10) and assume that for $k, \ell \in \mathbb{Z}$ with $|k-\ell| \leq 1$, we have

$$
\lim _{n \rightarrow \infty} \sup _{m=1, \ldots, N_{n}}\left|\left(\mathcal{J}_{m}\right)_{n+k, n+\ell}-a_{|k-\ell|}\left(t_{m}^{(n)}\right)\right|=0
$$

and

$$
\lim _{n \rightarrow \infty} \sup _{m=1, \ldots, N_{n}}\left|\frac{c_{n+\ell, m}}{c_{n+k, m}}-\mathrm{e}^{\tau\left(t_{m}^{(n)}\right)(k-\ell)}\right|=0 .
$$


Then for any function $g: I \times \mathbb{R} \rightarrow \mathbb{R}$ such that $g(t, x)$ is piecewise continuous in $t$ and a polynomial in $x$, we have that $Y_{n}(g)$ as defined in (2.24) satisfies

$$
Y_{n}(g)-\mathbb{E} Y_{n}(g) \rightarrow N\left(0, \sum_{k=1}^{\infty} \iint_{I \times I} \mathrm{e}^{-\left|\tau\left(t_{2}\right)-\tau\left(t_{1}\right)\right| k} k g_{k}\left(t_{1}\right) g_{-k}\left(t_{2}\right) \mathrm{d} t_{1} \mathrm{~d} t_{2}\right)
$$

as $n \rightarrow \infty$, with

$$
g_{k}(t)=\frac{1}{\pi} \int_{0}^{\pi} g\left(t, a_{0}(t)+2 a_{1}(t) \cos \theta\right) \cos k \theta \mathrm{d} \theta .
$$

PROOF. The proof follows from Theorem 2.7 in the same way as Corollary 2.4 followed from Theorem 2.3.

REMARK 2.5. By (2.22), we can write the variance also as

$$
\frac{1}{\pi} \sum_{k=1}^{\infty} \int \frac{k^{2}}{\omega^{2}+k^{2}}\left|\int_{I} \mathrm{e}^{-\mathrm{i} \omega \tau(t)} g_{k}(t) \mathrm{d} t\right|^{2} \mathrm{~d} \omega .
$$

This will be useful later on.

Again, Theorem 2.7 is stated for a function $g(t, x)$ that is a polynomial in $x$. Under similar conditions as in Theorem 2.5 we can extend this to a larger class of functions.

THEOREM 2.9. Assume that all the conditions of Corollary 2.8 hold. In addition, assume that there exists a compact set $E \subset \mathbb{R}$ such that either

(1) all supports $S\left(\mu_{m}^{(n)}\right) \subset E$ for $n \in \mathbb{N}$ and $m=1, \ldots, N$, or, more generally,

(2) for every $k \in \mathbb{N}$ and we have

$$
\sup _{m=1, \ldots, N_{n}} \int_{\mathbb{R} \backslash E}|x|^{k} K_{n, N}(m, x, m, x) \mathrm{d} \mu_{m}(x)=o(1 / n),
$$

as $n \rightarrow \infty$.

Moreover, assume that $t_{m}^{(n)}$ are such $N_{n} \sum_{m=1}^{N_{n}}\left(t_{m+1}^{(n)}-t_{m}^{(n)}\right)^{2}$ is bounded in $n$. Then Theorem 2.7 also holds wit for any $g$ such that $x \mapsto g(t, x)$ is a $C^{1}$ function growing at most polynomially at $\pm \infty$.

2.4. Connection to Gaussian Free Field. Finally, we discuss the relation of the above results with the Gaussian Free Field. We will focus on the situation of Theorem 2.7 and such that one of the conditions in Theorem 2.9 is valid, such that Theorem 2.7 holds for continuously differentiable $g$.

We will start by recalling the definition of the Gaussian Free Field without a detailed justification. More details and background can be found in the survey [45] and for a general reference on Gaussian Hilbert spaces we refer to [27]. 
Let $\mathcal{D}$ be a simply connected domain in $\mathbb{R}^{2}$. With this domain, we consider the space of test functions $\mathcal{H}_{\nabla}$ defined as follows: we start with space $C_{0}^{1}(\mathcal{D})$ of all continuously differentiable functions that vanish at the boundary of $\mathcal{D}$. On that space, we define the norm

$$
\|\phi\|_{\nabla}^{2}=\pi \iint_{\mathcal{D}}|\nabla \phi(w)|^{2} \mathrm{~d} m(w),
$$

where $\mathrm{d} m$ stands for the planar Lebesgue measure on $\mathcal{D}$. The space of test function $\mathcal{H}_{\nabla}$ is then defined as the closure of $C_{0}^{1}(\mathcal{D})$ with respect to this norm. Now that we have the space of test functions, we define a random generalized functions by providing how it acts on each test function. More precisely, we define the Gaussian Free Field to be a collection of random variables $\left\{\langle F, \phi\rangle_{\nabla}\right\}_{\phi}$ indexed by $\phi \in \mathcal{H}_{\nabla}$ such that

$$
\langle F, \phi\rangle_{\nabla} \sim N\left(0,\|\phi\|_{\nabla}^{2}\right)
$$

and such that $\phi \mapsto\langle F, \phi\rangle_{\nabla}$ is linear. That these two properties suffice for the definition is explained in Proposition 2.13 in [45] and the discussion around it. For instance, by linearity and the polarization identity, the correlation $\mathbb{E}\left[\left\langle F, \phi_{1}\right\rangle_{\nabla}\langle F\right.$, $\left.\phi_{2}\right\rangle_{\nabla}$ ] can be computed to be $\left(\phi_{1}, \phi_{2}\right)_{\nabla}$. Alternatively, the Gaussian Free Field can be introduced by justifying the formal series $F=\sum \alpha_{j} \phi_{j}$ where $\alpha_{j}$ are independent standard Gaussians and $\left\{\phi_{j}\right\}$ is any orthonormal basis for $\mathcal{H}_{\nabla}$.

Now let us first focus on the example given in the Introduction and let $h_{n}$ be the height function as defined in (1.1). The statement now is that the fluctuations of $h_{n}-\mathbb{E} h_{n}$ are described by the Gaussian Free Field in appropriately chosen coordinates. That is, there exists a simply connected domain $\mathcal{D}$ and a homeomorphism

$$
\Omega: \mathcal{D} \rightarrow \mathcal{E}: w=(\tau, \theta) \mapsto(t, x),
$$

where $\mathcal{E}$ is the ellipse (1.2), such that the push-forward of $h_{n}-\mathbb{E} h_{n}$ under the map $\Omega$ converges to the Gaussian Free Field on $\mathcal{D}$ with Dirichlet boundary conditions. That is,

$$
\left\langle h_{n} \circ \Omega, \phi\right\rangle_{\nabla}-\left\langle\mathbb{E} h_{n} \circ \Omega, \phi\right\rangle_{\nabla} \rightarrow N\left(0,\|\phi\|_{\nabla}^{2}\right),
$$

as $n \rightarrow \infty$, for some natural pairing $\left\langle h_{n} \circ \Omega, \phi\right\rangle_{\nabla}$. It is important to note that the Gaussian Free Field is a universal object; the coordinate transform is not and depends on the specific problem at hand.

The relation with linear statistics is explained as follows (see also [19]), which also gives the precise form of the pairing $\left\langle h_{n} \circ \Omega, \phi\right\rangle_{\nabla}$ that we will use. First, by integration by parts and a change of variables we obtain

$$
\begin{aligned}
& \pi \iint_{\mathcal{D}} \nabla h_{n}(\Omega(w)) \cdot \nabla \phi(w) \mathrm{d} m(w) \\
& \quad=-\pi \iint_{\mathcal{E}} h_{n}(x, t) \Delta \phi(w(t, x)) \frac{\mathrm{d}(\tau, \theta)}{\mathrm{d}(t, x)} \mathrm{d} x \mathrm{~d} t
\end{aligned}
$$


where $d(\tau, \theta) / d(t, x)$ stands for the Jacobian of the map $\Omega^{-1}$. We then use the fact that $h_{n}(t, x)=\sum_{j=1}^{n} \chi_{\left(-\infty, \gamma_{j}(t)\right]}(x)$ to rewrite the right-hand side as

$$
-\pi \int_{I} \sum_{j=1}^{n} \int_{-\infty}^{\gamma_{j}(t)} \Delta \phi(w(t, x)) \frac{\mathrm{d}(\tau, \theta)}{\mathrm{d}(t, x)} \mathrm{d} x \mathrm{~d} t .
$$

Finally, the pairing $\left\langle h_{n}, \phi\right\rangle_{\nabla}$ is then defined by a discretization of the integral over $t$,

$$
\begin{aligned}
\left\langle h_{n}, \phi\right\rangle_{\nabla}= & -\sum_{m=1}^{N} \frac{1}{t_{m+1}-t_{m}} \\
& \times \sum_{j=1}^{n} \pi \int_{-\infty}^{\gamma_{j}\left(t_{m}^{(n)}\right)} \Delta \phi\left(w\left(t_{m}, x\right)\right) \frac{\mathrm{d}(\tau, \theta)}{\mathrm{d}(t, x)} \mathrm{d} x .
\end{aligned}
$$

Now note that $\left\langle h_{n}, \phi\right\rangle_{\nabla}=Y_{n}(g)$ where $Y_{n}(g)$ is the linear statistic as in (2.24) with

$$
g(t, y)=-\pi \int_{-\infty}^{y} \Delta \phi(w(t, x)) \frac{\mathrm{d}(\tau, \theta)}{\mathrm{d}(t, x)} \mathrm{d} x .
$$

Hence the pairing of the height function with a test function, reduces to a linear statistic for the point process $\left\{\gamma_{j}\left(t_{m}\right)\right\}_{j=1, m=1}^{n, N}$ and we can apply Proposition 1.1 to find its limiting fluctuations, which leads to (2.29) as we will show below.

We will state the result in the more general setup of Theorem 2.9. That is, we consider a probability measure of the form in (2.1) satisfying Assumption 2.1 in the orthogonal polynomial situation (2.10). We also assume that the conditions of Corollary 2.8 are satisfied. That is, there exists interval $I=[\alpha, \beta]$ and functions $a_{0}: I \rightarrow \mathbb{R}, a_{1}: I \rightarrow \mathbb{R}$ and a function $\tau(t)$ such that we have the limits (2.26) and (2.27) for some partitioning $\left\{t_{m}^{(n)}\right\}$ of $I$. For simplicity, we will assume that $\tau$ is continuously differentiable and $\tau^{\prime}(t)>0$ for $t \in I$.

We thus obtain the random points $\left\{\left(m, x_{j, m}\right)\right\}_{j=1, m=1}^{n, N_{n}}$ and we ask for the fluctuations of the height function defined by

$$
h\left(t_{m}^{(n)}, x\right)=\#\left\{j \mid x_{j, m} \leq x\right\} .
$$

Then the region $\mathcal{E}$ and the coordinate transform $\Omega$ are determined by the function $\tau$ as follows. First, define

$$
\mathcal{E}=\left\{(t, x) \mid-2 a_{1}(t) \leq x-a_{0}(t) \leq 2 a_{1}(t)\right\} .
$$

Note that $\tau(t)$ is strictly increasing, and hence it has an inverse $t(\tau)$. Then, with

$$
\mathcal{D}=\{(\tau, \theta) \mid \tau \in(\tau(\alpha), \tau(\beta)), \theta \in(0, \pi)\},
$$

the map

$$
\Omega: \mathcal{D} \rightarrow \mathcal{E}:(\tau, \theta) \mapsto(t(\tau), x(\tau, \theta))=(t(\tau), 2 a(t(\tau)) \cos \theta),
$$


is a bijection and has the inverse

$$
\Omega^{-1}: \mathcal{E} \rightarrow \mathcal{D}:(t, x) \mapsto(\tau(t), \theta(t, x))=\left(\tau(t), \arccos \frac{x}{2 a(t)}\right) .
$$

In this setting, we have that the push-forward by $\Omega$ of the fluctuations of the height function $h_{n}$ are governed by the Gaussian Free Field on $\mathcal{D}$ in the following sense.

THEOREM 2.10. Let $\left\{\left(m, x_{j, m}\right)\right\}_{j, m=1}^{n, N}$ be random from a probability measure of the form (2.1) satisfying the conditions in Theorem 2.9 with the parameters as described above.

Let $\phi$ be a twice continuously differentiable real-valued function with compact support in $\mathcal{D}$ and consider the pairing

$$
\left\langle h_{n}, \phi\right\rangle_{\nabla}:=-\sum_{m=1}^{N} \frac{1}{t_{m+1}^{(n)}-t_{m}^{(n)}} \sum_{j=1}^{n} \pi \int_{-\infty}^{x_{j, m}} \Delta \phi(w(t, x)) \frac{\mathrm{d}(\tau, \theta)}{\mathrm{d}(t, x)} \mathrm{d} x .
$$

Then, as $n \rightarrow \infty$,

$$
\left\langle h_{n}, \phi\right\rangle_{\nabla}-\mathbb{E}\left[\left\langle h_{n}, \phi\right\rangle_{\nabla}\right] \rightarrow N\left(0,\|\phi\|_{\nabla}^{2}\right),
$$

in distribution.

PROOF. We start by recalling that $\left\langle h_{n}, \phi\right\rangle_{\nabla}$ is the linear statistic $Y_{n}(g)$ as in (2.24) with

$$
g(t, y)=-\pi \int_{-\infty}^{y} \Delta \phi(w(t, x)) \frac{\mathrm{d}(\tau, \theta)}{\mathrm{d}(t, x)} \mathrm{d} x .
$$

Note that $x \mapsto g(t, x)$ is a continuously differentiable and bounded function. Moreover, the point process satisfies the assumptions in Theorem 2.9 so that, as $n \rightarrow \infty$,

$$
\left\langle h_{n}, \phi\right\rangle_{\nabla}-\mathbb{E}\left[\left\langle h_{n}, \phi\right\rangle_{\nabla}\right] \rightarrow N\left(0, \sigma(g)^{2}\right),
$$

in distribution, with (see Remark 2.4)

$$
\sigma(g)^{2}=\frac{1}{\pi} \sum_{k=1}^{\infty} \int_{\mathbb{R}} \frac{k^{2}}{\omega^{2}+k^{2}}\left|\int_{I} \mathrm{e}^{-\mathrm{i} \tau(t) \omega} g_{k}(t) \mathrm{d} t\right|^{2} \mathrm{~d} \omega
$$

and

$$
g_{k}(t)=\frac{1}{\pi} \int_{0}^{\pi} g\left(t, a_{0}(t)+2 a_{1}(t) \cos \theta\right) \cos k \theta \mathrm{d} \theta .
$$

It remains to show that we can rewrite the variance so that it matches with the one in the statement.

We start by noting that the Jacobian for the map is given by

$$
\frac{\mathrm{d}(\tau, \theta)}{\mathrm{d}(t, x)}=\tau^{\prime}(t) \frac{\partial \theta}{\partial x} \text {. }
$$


Then, by a change of variables we have

$$
\begin{aligned}
g(t, y) & =\pi \int_{a_{0}(t)-2 a_{1}(t)}^{y} \Delta \phi(\tau(t), \theta(t, x)) \frac{\mathrm{d}(\tau, \theta)}{\mathrm{d}(t, x)} \mathrm{d} x \\
& =\pi \tau^{\prime}(t) \int_{a_{0}(t)-2 a_{1}(t)}^{y} \Delta \phi(\tau(t), \theta(t, x)) \frac{\partial \theta}{\partial x} \mathrm{~d} x \\
& =\pi \tau^{\prime}(t) \int_{0}^{\theta(t, y)} \Delta \phi(\tau(t), \theta) \mathrm{d} \theta
\end{aligned}
$$

Clearly, since $\Omega$ and $\Omega^{-1}$ are each others inverse maps, we have

$$
\theta\left(t, a_{0}(t)+2 a_{1}(t) \cos (t)\right)=\theta,
$$

and hence

$$
g\left(t, a_{0}(t)+2 a_{1}(t) \cos \theta\right)=\pi \tau^{\prime}(t) \int_{0}^{\theta} \Delta \phi(\tau(t), \tilde{\theta}) \mathrm{d} \tilde{\theta} .
$$

This implies that

$$
\begin{aligned}
k g_{k}(t) & =\frac{1}{\pi} \int_{0}^{\pi} g\left(t, a_{0}(t)+2 a_{1}(t) \cos \theta\right) k \cos k \theta \mathrm{d} \theta \\
& =\tau^{\prime}(t) \int_{0}^{\pi} \int_{0}^{\theta} \Delta \phi(\tau(t), \tilde{\theta}) \mathrm{d} \tilde{\theta} k \cos k \theta \mathrm{d} \theta \\
& =\tau^{\prime}(t) \int_{0}^{\pi} \Delta \phi(\tau(t), \theta) k \sin k \theta \mathrm{d} \theta
\end{aligned}
$$

using integration by parts in the last step. We then continue by inserting the last expressions and using the fact that $\phi$ has compact support in $\mathcal{D}$,

$$
\begin{aligned}
k \int_{I} \mathrm{e}^{-\mathrm{i} \tau(t) \omega} g_{k}(t) \mathrm{d} t & =\int_{I} \int_{0}^{\pi} \mathrm{e}^{-\mathrm{i} \tau(t) \omega} \Delta \phi(\tau(t), \theta) k \sin k \theta \mathrm{d} \theta \tau^{\prime}(t) \mathrm{d} t \\
& =\int_{\mathbb{R}} \int_{0}^{\pi} \mathrm{e}^{-\mathrm{i} \tau \omega} \Delta \phi(\tau, \theta) k \sin k \theta \mathrm{d} \theta \mathrm{d} \tau \\
& =\pi(\mathcal{G} \Delta \phi)(\omega, k),
\end{aligned}
$$

where $\mathcal{G}$ is the operator

$$
\mathcal{G} f(\omega, k)=\frac{1}{\pi} \int_{\mathbb{R}} \int_{0}^{\pi} f(\tau, \theta) \mathrm{e}^{-\mathrm{i} \omega \tau} \sin k \theta \mathrm{d} \theta \mathrm{d} \tau .
$$

Since $\left\{\sqrt{\frac{2}{\pi}} \sin k \theta\right\}_{k=1}^{\infty}$ is an orthonormal basis for $\mathbb{L}_{2}([0, \pi))$ and since the integral over $\tau$ is the usual Fourier transform [with normalization $(2 \pi)^{-1 / 2}$ ], we see that $\mathcal{G}$ defines a unitary transform from $\mathbb{L}_{2}(\mathcal{D})$ to $\mathbb{L}_{2}(\mathbb{R}) \times \ell_{2}(\mathbb{N})$. It is also easy to check 
that $\mathcal{G} \Delta \phi(\omega, k)=-\left(\omega^{2}+k^{2}\right) \mathcal{G} \phi(\omega, k)$. We then apply Plancherel's Theorem to write

$$
\begin{aligned}
& \frac{1}{\pi} \sum_{k=1}^{\infty} \int_{\mathbb{R}} \frac{k^{2}}{\omega^{2}+k^{2}}\left|\int_{I} \mathrm{e}^{-\mathrm{i} \tau(t) \omega} g_{k}(t) \mathrm{d} t\right|^{2} \mathrm{~d} \omega \\
& \quad=\pi \sum_{k=1}^{\infty} \int_{\mathbb{R}} \frac{|(\mathcal{G} \Delta \phi)(\omega, k)|^{2}}{\omega^{2}+k^{2}} \mathrm{~d} \omega=-\pi \sum_{k=1}^{\infty} \int_{\mathbb{R}} \overline{\mathcal{G} \Delta \phi(\omega, k)} \mathcal{G} \phi(\omega, k) \mathrm{d} \omega \\
& \quad=-\pi \iint_{\mathcal{D}} \phi \overline{\Delta \phi}=\pi \iint_{\mathcal{D}} \phi \Delta \phi=\|\phi\|_{\nabla}^{2},
\end{aligned}
$$

and this proves the statement.

3. Examples. In this section, we will illustrate the main results by discussing several examples.

3.1. Stationary non-colliding processes. The first class of examples is that of non-colliding processes for which the classical orthogonal polynomials ensembles are the invariant measures. The construction we will follow is a well-known approach using Doob's $h$-transform and the Karlin McGregor Theorem; see, for example, [34] for a discussion. An alternative way of defining the processes is to start with a generator for a single particle process and then define an $n$-particle process by constructing a generator on the space of symmetric functions $[10,42]$.

Suppose we are given a Markov process for a single particle and let us assume that it has a transition function $P_{t}(x, y) \mathrm{d} \mu(y)$ on a subset $E \subset \mathbb{R}$ that can be written as

$$
P_{t}(x, y) \mathrm{d} \mu(y)=\sum_{j=0}^{\infty} \mathrm{e}^{-\lambda_{j} t} p_{j}(x) p_{j}(y) \mathrm{d} \mu(y),
$$

where

$$
0=\lambda_{0}<\lambda_{1}<\lambda_{2}<\cdots
$$

and $p_{j}(x)$ are orthogonal polynomials with respect to $\mathrm{d} \mu(y)$. That is, $\left\{p_{k}\right\}_{k}$ is the unique family of polynomials such that $p_{k}$ is a polynomial of degree $k$ with positive leading coefficient and

$$
\int p_{k}(x) p_{\ell}(x) \mathrm{d} \mu(x)=\delta_{k \ell} .
$$

In other words, we assume that the generator for the Markov process has eigenvectors $p_{j}$ and eigenvalues $\lambda_{j}$. It is standard that the classical orthogonal polynomials appear in this way, as we will see.

We then construct a Markov process on the Weyl chamber

$$
\mathcal{W}_{n}=\left\{\left(x_{1}, \ldots, x_{n}\right) \in \mathbb{R}^{n} \mid x_{1}<\cdots<x_{n}\right\} .
$$


First, we note that by a theorem of Karlin McGregor it follows, under general conditions on the Markov process, that the joint probability distribution for the positions $y_{j}$ after time $t>0$ of particles that (1) each perform a single particle process given by $P_{t},(2)$ start at $x_{1}, \ldots, x_{n}$ and (3) are conditioned not to collide in $[0,1]$, is given by

$$
\operatorname{det}\left(P_{t}\left(x_{i}, y_{j}\right)\right)
$$

Then by (2.3) and (3.1) it follows that

$$
\begin{gathered}
\int_{\mathcal{W}_{n}} \operatorname{det}\left(P_{t}\left(x_{i}, y_{j}\right)\right) \operatorname{det}\left(p_{j-1}\left(y_{k}\right)\right)_{j, k=1}^{n} \mathrm{~d} \mu\left(y_{1}\right) \cdots \mathrm{d} \mu\left(y_{n}\right) \\
=\mathrm{e}^{-t \sum_{j=0}^{n-1} \lambda_{j}} \operatorname{det}\left(p_{j-1}\left(x_{k}\right)\right)_{j, k=1}^{n} .
\end{gathered}
$$

Moreover, $\operatorname{det}\left(p_{j-1}\left(x_{k}\right)\right)_{j, k=1}^{n}=c \prod_{1 \leq i<j \leq n}\left(x_{j}-x_{i}\right)$ is positive. Hence it is a positive harmonic function and we can apply Doob's $h$-transform to arrive at the transition function

$$
P_{t}(\vec{x}, \vec{y})=\mathrm{e}^{t \sum_{j=0}^{n-1} \lambda_{j}} \operatorname{det}\left(P_{t}\left(x_{i}, y_{j}\right)\right)_{i, j=1}^{n} \frac{\operatorname{det}\left(p_{j-1}\left(y_{k}\right)\right)_{j, k=1}^{n}}{\operatorname{det}\left(p_{j-1}\left(x_{k}\right)\right)_{j, k=1}^{n}} .
$$

This defines the Markov process on $\mathcal{W}_{n}$ that we will be interested in. Finally, it is not hard to show from (2.3) that the unique invariant measure is given by the orthogonal polynomial ensemble [34],

$$
\left(\operatorname{det}\left(p_{j-1}\left(x_{k}\right)\right)_{j, k=1}^{n}\right)^{2} \mathrm{~d} \mu\left(x_{1}\right) \cdots \mathrm{d} \mu\left(x_{n}\right) .
$$

In other words, the above construction provides a way for defining a stochastic dynamics for which the classical orthogonal polynomial ensembles are the invariant measures.

We consider this Markov process in the stationary situation. That is, we fix $t_{1}<t_{2}<\cdots<t_{N} \in \mathbb{R}$ and start the Markov process with the invariant measure at $t_{1}$. Then we obtain a probability measure for the locations at $t_{m}$

$$
\begin{aligned}
& \operatorname{det}\left(p_{j-1}\left(x_{k}\left(t_{1}\right)\right)\right)_{j, k=1}^{n} \prod_{m=1}^{N-1} \operatorname{det}\left(P_{t_{m+1}-t_{m}}\left(x_{j}\left(t_{m}\right), x_{k}\left(t_{m+1}\right)\right)\right)_{j, k=1}^{n} \\
& \quad \times \operatorname{det}\left(p_{j-1}\left(x_{k}\left(t_{N}\right)\right)\right)_{j, k=1}^{n} \prod_{m=1}^{N} \prod_{j=1}^{n} \mathrm{~d} \mu\left(x_{j}\left(t_{m}\right)\right),
\end{aligned}
$$

which, after a symmetrization, is exactly of the form (2.1). In fact, it is an example of an orthogonal polynomial situation as given in (2.10) with $c_{j, m}=\mathrm{e}^{-t_{m} \lambda_{j}}$. Before we apply Corollary 2.4 , we recall that the orthogonal polynomials on the real line are subject to a three term recurrence relation

$$
x p_{k}(x)=a_{k+1} p_{k+1}(x)+b_{k} p_{k}(x)+a_{k} p_{k-1}(x),
$$


for some numbers $b_{k} \in \mathbb{R}$ and $a_{k}>0$. We recall that we allow the measure $\mu$ to vary with $n$ so that also $b_{k}$ and $a_{k}$ may vary with $n$, and hence we will write $a_{k}=a_{k}^{(n)}$ and $b_{k}^{(n)}$.

THEOREM 3.1. Suppose that for some $a>0$ and $b \in \mathbb{R}$ we have

$$
\begin{aligned}
& \lim _{n \rightarrow \infty} a_{n+k}^{(n)}=a, \\
& \lim _{n \rightarrow \infty} b_{n+k}^{(n)}=b,
\end{aligned}
$$

for $k \in \mathbb{Z}$. Then let $t_{1}<\cdots<t_{N} \in \mathbb{R}$ be such that, for some $\tau_{j}$ and sequence $\kappa_{n}$,

$$
\lim _{n \rightarrow \infty} \kappa_{n}\left(\lambda_{n+k}-\lambda_{n+l}\right) t_{j}=(k-l) \tau_{j}
$$

as $n \rightarrow \infty$. Then for any $f:\{1, \ldots, N\} \times \mathbb{R}$ such that $f(m, x)$ is a polynomial, we have

$$
X_{n}(f)-\mathbb{E} X_{n}(f) \rightarrow N\left(0, \sum_{m_{1}, m_{2}=1}^{N} \sum_{k=1}^{\infty} k \mathrm{e}^{-\left|\tau_{m_{1}}-\tau_{m_{2}}\right| k} f_{k}^{\left(m_{1}\right)} f_{k}^{\left(m_{2}\right)}\right),
$$

where

$$
f_{k}^{(m)}=\frac{1}{\pi} \int_{0}^{\pi} f(m, 2 a \cos \theta+b) \cos k \theta \mathrm{d} \theta
$$

Moreover, for any $g: I \times \mathbb{R} \rightarrow \mathbb{R}$ such that $g(t, x)$ is a polynomial in $x$ we have that $Y_{n}(g)$ as defined in (2.24) satisfies

$$
Y_{n}(g)-\mathbb{E} Y_{n}(g) \rightarrow N\left(0, \sum_{k=1}^{\infty} \iint_{I \times I} \mathrm{e}^{-\left|\tau\left(t_{2}\right)-\tau\left(t_{1}\right)\right| k} k g_{k}\left(t_{1}\right) g_{-k}\left(t_{2}\right) \mathrm{d} t_{1} \mathrm{~d} t_{2}\right)
$$

as $n \rightarrow \infty$, with

$$
g_{k}(t)=\frac{1}{\pi} \int_{0}^{\pi} g\left(t, a_{0}+2 a_{1} \cos \theta\right) \cos k \theta \mathrm{d} \theta .
$$

PROOF. This is a direct consequence of Corollaries 2.4 and 2.8, with $c_{j, m}=$ $\mathrm{e}^{-t_{m} \lambda_{j}}$

The point is now that for the Markov process related to the classical polynomials we can easily verify the stated condition by looking up the explicit values of the parameters in standard reference works on classical orthogonal polynomials, such as [33].

We will now illustrate the results with some examples. To start with, we consider the classical Hermite, Laguerre and Jacobi polynomials, which are well known to be eigenfunctions for a second order differential operator that can be used as the generator for the Markov process. 

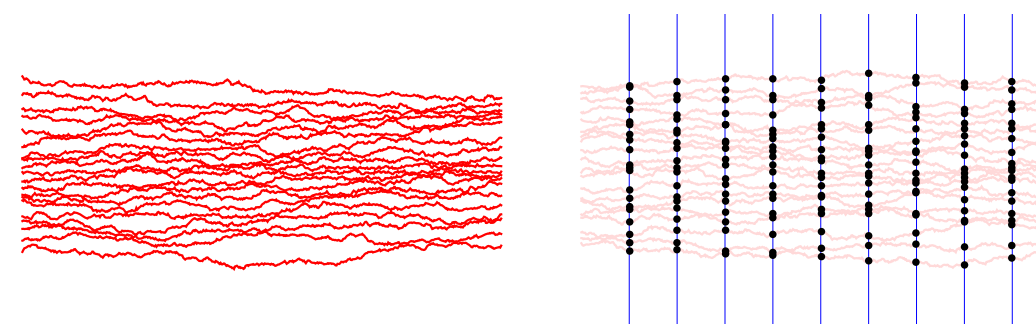

FIG. 2. The left figure shows a sampling from a stationary non-colliding process generated by the Ornstein-Uhlenbeck process of size $n=20$. At the right, we intersect the trajectories at multiple times $t_{m}$.

EXAMPLE 3.2 (Non-colliding Ornstein-Uhlenbeck processes). Let us start where we take $P_{t}(x, y)$ according to the Ornstein-Uhlenbeck process. This is the model that was considered by Dyson [22] for $\beta=2$. See Figure 2. In that case, we have

$$
P_{t}(x, y) \mathrm{d} \mu(y)=\frac{1}{\sqrt{2 \pi\left(1-\mathrm{e}^{-2 t}\right)}} \mathrm{e}^{-\frac{\left(\mathrm{e}^{-t} x-y\right)^{2}}{2\left(1-\mathrm{e}^{-2 t}\right)}} \mathrm{d} y,
$$

as the transition function. By Mehler's formula for the Hermite polynomials, this can be expanded as

$$
P_{t}(x, y) \mathrm{d} \mu(y)=\sum_{j=0}^{\infty} \mathrm{e}^{-j t} H_{j}(x) H_{j}(y) \mathrm{e}^{-y^{2} / 2} \mathrm{~d} y,
$$

where $H_{j}(y)$ are the normalized Hermite polynomials where the orthogonality is with respect to $\mathrm{e}^{-y^{2} / 2} \mathrm{~d} y$ on $\mathbb{R}$.

The Hermite polynomials satisfy the recurrence

$$
x H_{k}(x)=\sqrt{k+1} H_{k+1}(x)+\sqrt{k} H_{k-1}(x) .
$$

The recurrence coefficients grow and in order to get a meaningful limit we need to rescale the process. Indeed, when $n \rightarrow \infty$ the paths at any given time $t$ fill the interval $(-2 \sqrt{n}, 2 \sqrt{n})$ and we rescale the space variable and introduce the new variable $\xi$ by

$$
x=\sqrt{n} \xi .
$$

Then the rescaled orthonormal polynomials are $p_{k}(\xi)=n^{-1 / 4} H_{k}(\sqrt{n} \xi)$ and for these polynomials we have the following recursion

$$
p_{k}(\xi)=\sqrt{\frac{k+1}{n}} p_{k+1}(\xi)+\sqrt{\frac{k}{n}} p_{k-1}(\xi) .
$$

One readily verifies that

$$
a_{n+k}^{(n)} \rightarrow 1, \quad b_{n+k}^{(n)}=0 .
$$


Moreover, since $\lambda_{j}=j$, we also have

$$
\left(\lambda_{n+k}-\lambda_{n+\ell}\right) t_{j}=(k-\ell) t_{j}
$$

Therefore, the conditions of Theorem 3.1 are satisfied with $a=1, b=0$ and $\tau_{j}=$ $t_{j}$. In fact, for the Hermite polynomials one can verify that the second condition in Theorem 2.5 is satisfied, and hence Theorem 3.1 also holds for function $f$ : $\{1, \ldots, N\} \times \mathbb{R}$ such that $x \rightarrow f(m, x)$ is a continuously differentiable function that grows at most polynomially at $\pm \infty$. This follows, for example, after a classical steepest decent analysis on the integral representation of the Hermite polynomials or by a Riemann-Hilbert analysis. We will leave the tedious details to the reader.

Finally, we note that the non-colliding Brownian bridges model from the Introduction can be obtained from the above model after the change of variables

$$
\left(\begin{array}{l}
t \\
\xi
\end{array}\right) \mapsto\left(\begin{array}{c}
\frac{1}{1+\mathrm{e}^{-t}} \\
\frac{\xi}{\cosh t}
\end{array}\right)
$$

This is discussed in, for example, [30] and we refer to that paper for more details. This also proves Proposition 1.1.

EXAMPLE 3.3 (Non-colliding Jacobi diffusions). The next example is that of Jacobi diffusions, which have also been discussed in $[18,26]$. For $\alpha, \beta>-1$, consider the Jacobi diffusion [44]

$$
P_{t}(x, y) \mathrm{d} \mu(y)=\sum_{j=1}^{\infty} \mathrm{e}^{-j(j+\alpha+\beta+1)} p_{j}^{\alpha, \beta}(x) p_{j}^{\alpha, \beta}(y) y^{\alpha}(1-y)^{\beta} \mathrm{d} y
$$

on $[0,1]$, where $p_{j}^{(\alpha, \beta)}(x)$ is the polynomial of degree $j$ with positive leading coefficient satisfying

$$
\int_{0}^{1} p_{j}^{(\alpha, \beta)}(x) p_{k}^{(\alpha, \beta)}(x) x^{\alpha}(1-x)^{\beta} \mathrm{d} x=\delta_{j k} .
$$

Also in this case, the recurrence coefficient are explicit. Without giving them, we mention that it can easily be computed that

$$
a_{n+k} \rightarrow \frac{1}{4}, \quad b_{n+k} \rightarrow 1 / 2
$$

In fact, these limits hold for any measure $w(x) \mathrm{d} x$ on $[0,1]$ with positive weight $w(x)>0$ (and even more general, the Denisov-Rakhmanov Theorem [47], Theorem 1.4.2, says that it holds for any general measure for which the essential support is $[0,1]$ and for which the density of the absolutely continuous part is strictly positive on $(0,1))$. 
In this case, the $\lambda_{j}=j(j+\alpha+\beta+1)$ is quadratic. For that reason, we will consider times $t_{j}=n(\alpha+\beta+2) \tau_{j}$ for some fixed $\tau_{j}$ 's, so that we have

$$
\kappa_{n} t_{j}\left(\lambda_{n+k}-\lambda_{n+\ell}\right)=\tau_{j}(k-\ell),
$$

with $\kappa_{n}=\frac{1}{n(\alpha+\beta+2)}$, and hence both conditions in Theorem 3.1 are satisfied with $a=1 / 4$ and $b=1 / 2$.

EXAMPLE 3.4 (Non-colliding squared radial Ornstein-Uhlenbeck processes). In the next example, we replace the Ornstein-Uhlenbeck process with its squared radial version. That is,

$$
P_{t}(x, y) \mathrm{d} \mu(y)=\frac{1}{1-\mathrm{e}^{-t}}\left(\frac{y}{x \mathrm{e}^{-t}}\right)^{r / 2} \mathrm{e}^{-\frac{x \mathrm{e}^{-t}}{1-\mathrm{e}^{-t}}-\frac{y}{1-\mathrm{e}^{-t}}} I_{r}\left(\frac{2 \sqrt{\mathrm{e}^{-t} x y}}{1-\mathrm{e}^{t}}\right)
$$

on $[0, \infty)$ where $r>-1$ is a parameter and $I_{r}$ stands for the modified Bessel function of the first kind of order $r$. The squared radial Ornstein-Uhlenbeck process is related to the squared Bessel process in a similar way as the Ornstein-Uhlenbeck process is to Brownian motion. Indeed, the squared Bessel process can be obtained by a change of variables. The latter process has been studied in the literature in the context of non-colliding processes before. In [35], it was used to define a dynamic version of the Laguerre ensemble from Random Matrix Theory. See Figure 3 for a sample.

Note that we can expand the transition function as [44]

$$
P_{t}(x, y) \mathrm{d} \mu(y)=\sum_{j=1}^{\infty} \mathrm{e}^{-j t} \frac{j !}{\Gamma(j+r+1)} L_{j}^{(r)}(x) L_{j}^{(r)}(x) y^{r} \mathrm{e}^{-y} \mathrm{~d} y,
$$

where $L_{j}^{(r)}(x)$ is the generalized Laguerre polynomial of degree $r$ (with orthogonality with respect to $y^{r} \mathrm{e}^{-y} \mathrm{~d} y$ ). These polynomials satisfy the recursive relation

$$
x L_{k}^{(r)}=-(k+1) L_{k+1}^{(r)}(x)+(2 k+r+1) L_{k}^{(r)}(x)-(k+r) L_{k-1}^{(r)}(x) .
$$
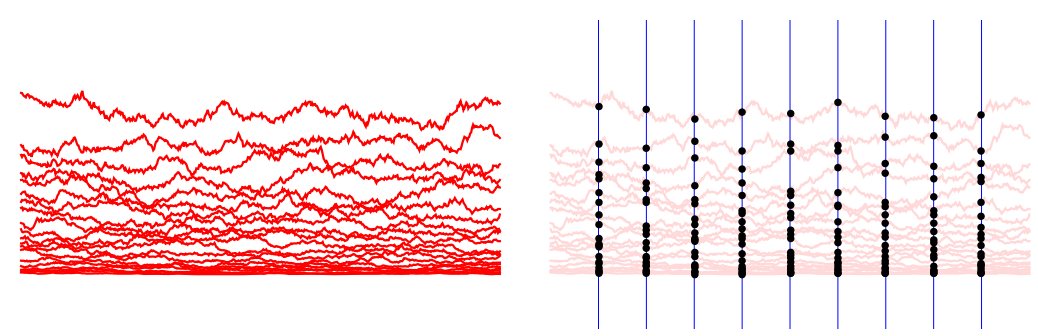

FIG. 3. The left figure shows a sampling from a stationary non-colliding process generated by the squared radial Ornstein-Uhlenbeck process of size $n=20$. At the right, we intersect the trajectories at multiple times $t_{m}$. 
Note that the recursion coefficients are growing, which means that we need to rescale the process. Moreover, the Laguerre polynomials are not normalized. Using an appropriate normalization and rescaling, it is straightforward to show that the conditions of Theorem 3.1 are satisfied with $a=1, b=2$ and $\tau_{j}=t_{j}$. To the best of our knowledge, we believe that this example is a new result that has not appeared before. It is also possible to prove the conditions of Theorems 2.5 and 2.9.

The three examples above can also be obtained from stochastic evolution for matrices. See [18, 22, 35] for more details. They are continuous in time and space. The next examples are concerned with a discrete space variable, based on birth and death processes. These are processes that model a population that can increase or decrease by one. By tuning the birth/death rates (which may depend on the size of the population), one obtains classical orthogonal polynomials of a discrete variable. We refer to [44] for more details and background. In the $n$-particle construction as before, we then arrive at stochastic evolution for which the classical discrete orthogonal polynomial ensembles are the invariant measures. We emphasize that there are other constructions [40] that lead to discrete orthogonal polynomial ensembles, such as the Charlier processes. Although there may be relations, these examples should not be confused with each other.

EXAMPLE 3.5 (Non-colliding Meixner Ensemble). In the first example, we start with a birth and death process on $\{0,1, \ldots\}$ with birth $\mu(n+\gamma)$ and death rate $n$, where $n$ is the size of the population. This process has the transition function

$$
P_{t}(x, y) \mathrm{d} \mu(y)=\sum_{j=0}^{\infty} \mathrm{e}^{-j t} \frac{\mu^{j}(\gamma)_{j}}{j !} M_{j}(x ; \gamma, \mu) M_{j}(y ; \gamma, \mu)(1-\mu)^{\gamma} \frac{\mu^{y}(\gamma)_{y}}{y !}
$$

on $\{0,1,2,3, \ldots\}$. Here, $(\gamma)_{y}$ denotes the Pochhammer symbol and $M_{j}$ is the Meixner polynomial of degree $j$.

The associated $n$-particle generalization appeared in [10]. We now show how the conditions of Theorem 3.1 are met.

The Meixner polynomials satisfy the following recursion

$$
\begin{aligned}
x M_{k}(x ; \gamma, \mu)= & -\frac{\mu(k+\gamma)}{1-\mu} M_{k+1}(x, \gamma, \mu) \\
& +\frac{k(1+\mu)+\mu \gamma}{1-\mu} M_{k}(x, \gamma, \mu) \\
& -\frac{k}{1-\mu} M_{k-1}(x, \gamma, \mu) .
\end{aligned}
$$

Also in this case, both a rescaling and normalization are needed. We define

$$
p_{k}(\xi)=(-1)^{k} \sqrt{\frac{\mu^{k}(\gamma)_{k}(1-\mu)^{\gamma}}{k !}} M_{k}(\xi n ; \gamma, \mu) \text {. }
$$


Then the recursion turns into

$$
\begin{aligned}
x p_{k}(\xi)= & \frac{\sqrt{\mu(k+\gamma)(k+1)}}{n(1-\mu)} p_{k+1}(\xi)+\frac{k(1+\mu)+\mu \gamma}{1-\mu} p_{k}(\xi) \\
& +\frac{\sqrt{\mu(\gamma+k-1) k}}{n(1-\mu)} p_{k-1}(\xi) .
\end{aligned}
$$

Now it easily follows that

$$
a_{n+k}^{(n)} \rightarrow \frac{\sqrt{\mu}}{1-\mu}, \quad b_{n+k}^{(n)} \rightarrow \frac{1+\mu}{1-\mu} .
$$

Also $\lambda_{j}$ so we have $\tau_{j}=t_{j}$. This shows that the conditions of Theorem 3.1 hold.

EXAMPLE 3.6 (Non-colliding Charlier). In the next example, we consider a birth and death process on $\{0,1, \ldots\}$ with birth $\mu$ and death rate $n$, where $n$ is the size of the population. This process has the transition function

$$
P_{t}(x, y) \mathrm{d} \mu(y)=\sum_{j=0}^{\infty} \mathrm{e}^{-j t} \frac{\mu^{j}}{j !} C_{j}(x ; \mu) C_{j}(y ; \mu) \mathrm{e}^{-\mu} \frac{\mu^{k}}{k !}
$$

for $x, y \in\{0,1,2,3, \ldots\}$, where $C_{j}(x ; \mu)$ is the Charlier polynomial of degree $j$.

To apply Theorem 3.1 for the corresponding $n$-particles process, we recall that the recursion for the Charlier reads

$$
x C_{k}(x ; \mu)=-\mu C_{k+1}(x ; \mu)+(k+\mu) C_{k}(x ; \mu)-k C_{k-1}(x ; \mu) .
$$

As before, we renormalize

$$
p_{k}(x)=(-1)^{k} \sqrt{\frac{\mu^{k}}{k !}} C_{k}(x ; \mu)
$$

which gives the new recurrence

$$
x p_{k}(x)=\sqrt{\mu(k+1)} p_{k}(x)+(k+\mu) p_{k}(x)+\sqrt{\mu k} p_{k-1}(x) .
$$

Now note that this case is special, since the $a_{k}$ 's and $b_{k}$ 's grow with different rates. This is a well-known feature of the Charlier polynomials. It means that there are two ways to get an interesting limit, which we will treat separately.

In the first one, we shift and rescale the space variable according to $\xi=$ $(x-n) / \sqrt{n}$, and set $\hat{p}_{k}(\xi)=n^{1 / 4} p_{k}(n+\sqrt{n} x)$. These polynomials satisfy the recurrence

$$
\hat{p}_{k}(\xi)=\sqrt{\frac{\mu(k+1)}{n}} \hat{p}_{k+1}(\xi)+\frac{k-n+\mu}{\sqrt{n}} p_{k}(\xi)+\sqrt{\frac{\mu k}{n}} p_{k-1}(\xi) .
$$

Hence we see that

$$
a_{n+k}^{(n)} \rightarrow \sqrt{\mu} \text { and } \quad b_{n+k}^{(n)} \rightarrow 0 .
$$


Combining this with the fact $\lambda_{j}=j$, we see that in this way the conditions of Theorem 3.1 are met.

In the second way, we allow $\mu$ to vary with $n$ and write $\mu=\tilde{\mu} n$. Then we consider the new variable $\xi=x / n$, and set $\tilde{p}_{k}(\xi)=\sqrt{n} \tilde{p}_{k}(x n)$. Now the recurrence becomes

$$
\tilde{p}_{k}(\xi)=\frac{\sqrt{\mu k(k+1)}}{n} \tilde{p}_{k+1}(\xi)+\frac{k(1+\mu)}{n} \tilde{p}_{k}(\xi)+\frac{\sqrt{\mu} k}{n} \tilde{p}_{k-1}(\xi) .
$$

In this case, we have

$$
a_{n+k}^{(n)} \rightarrow \sqrt{\mu} \quad \text { and } \quad b_{n+k}^{(n)} \rightarrow 1+\mu .
$$

Combining this again with the fact $\lambda_{j}=j$, we see that also in this way the conditions of Theorem 3.1 are met.

Finally, we want to mention that this process is different from what is usually referred to as the Charlier process $[36,41]$, which is non-colliding Poisson random walks starting from densely packed initial points. In that case, we only allow for up jumps. The Charlier Ensemble appears there as the fixed time distribution, but not as the invariant measure.

EXAMPLE 3.7 (Non-colliding Krawtchouck). In the final example, we consider a birth and death process on $\{0,1, \ldots, M\}$ with birth rate $p(M-n)$ and death rate $n(1-p)$, where $n$ is the size of the population, $p \in(0,1)$ and $M \in \mathbb{N}$. We then have the transition function

$$
\begin{aligned}
P_{t}(x, y) \mathrm{d} \mu(y) & \\
= & \sum_{j=0}^{M} \mathrm{e}^{-j t}\left(\begin{array}{c}
M \\
j
\end{array}\right) p^{j}(1-p)^{M-j} \\
& \times K_{j}(x ; M, p) K_{j}(y ; M, p)\left(\begin{array}{c}
M \\
k
\end{array}\right) p^{k} 1(-p)^{M-k}
\end{aligned}
$$

on $\{0,1,2,3, \ldots, M\}$, where $K_{j}$ is the Krawtchouk polynomial of degree $j$.

In order for the $n$-particles process to make sense, we need enough available nodes for all the paths. That is, we need a $M \geq n$. In fact, when taking the limit $n \rightarrow \infty$, we will assume that $M_{n}$ also goes to infinity such that

$$
\frac{M_{n}}{n} \rightarrow \gamma>1 \text {. }
$$

The Krawtchouk polynomial satisfy the recurrence

$$
\begin{aligned}
x K_{k}(x ; M, p)= & p(M-k) K_{k+1}(x ; M, p) \\
& -(p(M-k)+k(1-p)) K_{k}(x ; M, p) \\
& +k(1-p) K_{k-1}(x ; M, p) .
\end{aligned}
$$


In this case, we define the rescaled and normalized polynomials

$$
p_{k}(x)=(-1)^{k}\left(\begin{array}{c}
M \\
k
\end{array}\right)^{1 / 2}\left(\frac{p}{1-p}\right)^{k / 2}(-1)^{k} K_{k}(x ; M, p),
$$

and for these polynomials we get the recursion

$$
\begin{aligned}
x p_{k}(x)= & \sqrt{p(1-p)} \frac{\sqrt{(k+1)(M-k)}}{n} p_{k+1}(x)+\frac{p M-2 p k+k}{n} p_{k}(x) \\
& +\sqrt{p(1-p)} \frac{\sqrt{k(M-k+1)}}{n} p_{k-1}(x) .
\end{aligned}
$$

Hence in this model we have, with $M / n \rightarrow \gamma$,

$$
a_{n+k, n}^{(n)} \rightarrow \sqrt{p(1-p) \gamma}, \quad b_{n+k, n}^{(n)} \rightarrow p \gamma-2 p+1,
$$

and, again $\lambda_{j}=j$, so that the conditions of Theorem 3.1 are satisfied.

The invariant measure for the $n$-particle process is the Krawtchouk Ensemble. This ensemble also appears in random domino tilings of an Aztec diamond [29]. However, the multi-time processes here is different from the extended Krawtchouk Ensemble in [29]. It is also different form the process introduced in [36] for which the single time distribution is a Krwatchouk ensembles.

3.2. Nonstationary example. We now discuss a nonstationary example. An interesting feature of this example, is that the biorthogonal families do no longer consists of classical special functions. As a consequence, the correlation kernel cannot be expressed in terms of a double integral formula and its asymptotics is a challenging problem. In some special cases, this has been carried out by the author [21] using a $4 \times 4$ Riemann-Hilbert problem. Hence, proving Central Limit Theorems from the asymptotics of the correlation kernel (as in, e.g., [8, 19, 37]) seems difficult. However, the benefit of our method is that in these cases the conditions on the recurrence coefficients can still be checked with relative ease.

Consider the same construction ideas as the non-colliding Ornstein-Uhlenbeck process of Example 3.2, but instead of having the invariant measure as initial condition, we take the initial points random from a Unitary Ensemble. That is, we take $x_{j}$ random from the probability measure on $\mathbb{R}^{n}$ proportional to

$$
\prod_{1 \leq i<j \leq n}\left(x_{i}-x_{j}\right)^{2} \mathrm{e}^{-n \sum_{j=1}^{n} V\left(x_{j}\right)} \mathrm{d} x_{1} \cdots \mathrm{d} x_{n},
$$

where $V$ is a polynomial of even degree and positive leading coefficient (so that the above measure is indeed of finite mass). See also Figure 4. Then if we start the non-colliding Ornstein-Uhlenbeck process from these initial points and look at the positions $\left\{x_{j, m}\right\}_{j=1, m=1}^{n, N}$ at times

$$
0=t_{1}<\cdots<t_{N}
$$




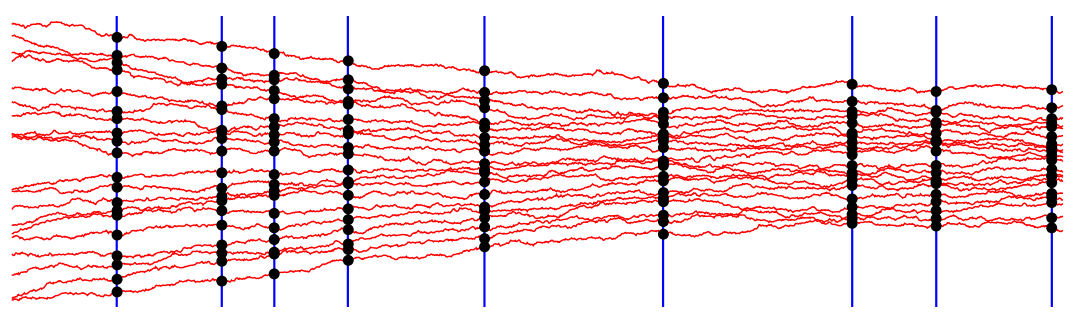

FIG. 4. The non-colliding Ornstein-Uhlenbeck process started from arbitrary points at $t=0$. In the example of Section 3.2, we take those initial points randomly from a Unitary Ensemble.

then we find that the following joint probability for these locations is proportional to

$$
\begin{gathered}
\operatorname{det}\left(x_{k, 1}^{j-1}\right)_{j, k=1}^{n} \prod_{m=1}^{N} \operatorname{det}\left(T_{m}\left(x_{j, m}, x_{k, m+1}\right)\right)_{j, k=1}^{n} \\
\times \operatorname{det}\left(x_{k, m}^{j-1}\right)_{j, k=1}^{n} \prod_{m=1}^{N} \prod_{j=1}^{n} \mathrm{~d} \mu_{m}\left(x_{j, m}\right),
\end{gathered}
$$

where, with $\Delta_{m}=\mathrm{e}^{-\left(t_{m+1}^{(n)}-t_{m}^{(n)}\right)}$

$$
\begin{aligned}
T_{m}(x, y) & =(2 \pi)^{-1 / 2} \exp \left(\frac{-n \Delta_{m}^{2}\left(x^{2}+y^{2}\right)+n \Delta_{m} x y}{1-\Delta_{m}^{2}}\right) \\
& =\sum_{j=0}^{\infty} \mathrm{e}^{-j t} H_{j}(\sqrt{n} x) H_{j}(\sqrt{n} y)
\end{aligned}
$$

and

$$
\mathrm{d} \mu_{m}(x)= \begin{cases}\mathrm{e}^{-n V(x)} \mathrm{d} x, & m=1, \\ \mathrm{e}^{-n x^{2} / 2} \mathrm{~d} x, & m=2, \ldots, N .\end{cases}
$$

(Note that we have rescaled space immediately.)

The functions in the determinant are not in the right form, as Assumption 2.1 is not yet satisfied. Hence the first thing to do is to rewrite the probability density. We start by defining $p_{j, n}$ to be the normalized orthogonal polynomial (with positive leading coefficient) with respect to $\mathrm{e}^{-n V(x)} \mathrm{d} x$. Now define

$$
\phi_{j, 1}(x)=p_{j-1, n}(x),
$$

and $\phi_{j, m}=\mathcal{T}_{m-1} \cdots \mathcal{T}_{1} \phi_{j, 1}$, for $m=2, \ldots, N$. To define the $\psi_{j, N}$ 's we first expand $p_{j-1, n}$ in terms of Hermite functions

$$
p_{j, n}(x)=\sum_{k=0}^{j} c_{j, k} H_{k}(x),
$$


where $H_{n}(x)$ are the normalized Hermite polynomials. We then define

$$
\psi_{j, N}(x)=\sum_{k=0}^{j-1} c_{j, k} \mathrm{e}^{k t_{N}} H_{k}(x) .
$$

It is then straightforward to check that

$$
\psi_{j, m}(x)=\mathcal{T}_{m}^{*} \cdots \mathcal{T}_{N-1}^{*} \psi_{j, N}(x)=\sum_{k=0}^{j-1} c_{j, k} \mathrm{e}^{k t_{m}} H_{k}(x) .
$$

Hence, we also have $\phi_{j, 1}(x)=\psi_{j, 1}(x)=p_{j-1, n}(x)$ and the bi-orthogonality condition is satisfied. In the following lemma, we show that these indeed are biorthogonal families and that they satisfy a recursion.

LEMMA 3.8. The $\phi_{j, m}$ satisfy a recurrence relation

$$
x\left(\begin{array}{c}
\phi_{1, m}(x) \\
\phi_{2, m}(x) \\
\vdots
\end{array}\right)=\mathbb{J}_{m}\left(\begin{array}{c}
\phi_{1, m}(x) \\
\phi_{2, m}(x) \\
\vdots
\end{array}\right)
$$

with

$$
(\mathbb{J})_{j, k}= \begin{cases}\mathrm{e}^{-t_{m}}(\mathcal{J})_{j, k}, & \text { if } j>k, \\ \mathrm{e}^{-t_{m}}(\mathcal{J})_{j, k}+2\left(V^{\prime}(\mathcal{J})\right)_{j, k} \sinh \left(t_{m}\right), & \text { if } j \leq k,\end{cases}
$$

where $\mathcal{J}$ is the Jacobi matrix associated to the polynomials $p_{j}$.

REMARK 3.1. Note that $\mathcal{J}$ is a banded matrix and $V$ is assumed to be a polynomial. Hence the matrix $V^{\prime}(\mathcal{J})$ that one obtains after inserting $\mathcal{J}$ into $V^{\prime}$ is welldefined and banded.

PROOF. For $m=1$, the statement is trivial since then $\psi_{j, 1}=\phi_{j, 1}=p_{j-1, n}$ and the recurrence matrix is $\mathcal{J}$ by definition, which is also the result when we substitute $t_{m}=0$ in the statement.

So it remains to deal with the case $m>1$. We first claim that

$$
p_{j-1, n}^{\prime}(x)=n \sum_{k<j}\left(V^{\prime}(\mathcal{J})\right)_{k, j} p_{k-1, n}(x) .
$$

To see this, we note that $p_{j-1, n}^{\prime}(x)$ is a polynomial of degree $j-2$, and hence it can be expanded in terms of the polynomials $p_{k-1, n}$ for $k=0, \ldots, j-1$. That the coefficients in the expansion are indeed as stated follows from an integration by 
parts

$$
\begin{aligned}
\int p_{k-1, n}(x) p_{j-1, n}^{\prime}(x) \mathrm{e}^{-n V(x)} \mathrm{d} x \\
=n \int V(x) p_{k-1, n}(x) p_{j-1, n}(x) \mathrm{e}^{-n V(x)} \mathrm{d} x \\
\quad-\int p_{k-1, n}^{\prime}(x) p_{j-1, n}(x) \mathrm{e}^{-n V(x)} \mathrm{d} x \\
=\left(V^{\prime}(\mathcal{J})\right)_{k, j},
\end{aligned}
$$

where the second integral in the middle part vanishes by orthogonality and the fact that $k<j$.

Then, for $m>1$, we have

$$
\phi_{j, m}(x)=\frac{1}{\sqrt{2 \pi\left(1-\mathrm{e}^{\left.-2 t_{m}\right)}\right.}} \int p_{j-1, n}(y) \mathrm{e}^{-n\left(V(y)+\frac{\mathrm{e}^{-2 t_{m}}\left(x^{2}+y^{2}\right)-2 \mathrm{e}^{-t_{m}} x y}{2\left(1-\mathrm{e}^{\left.-2 t_{m}\right)}\right.}\right)} \mathrm{d} y .
$$

Hence, by integration by parts,

$$
\begin{aligned}
x \phi_{j, m}(x)= & -\frac{2 \sinh t_{m}}{n} \mathrm{e}^{-\frac{n \mathrm{e}^{-2 t_{m} x^{2}}}{2\left(1-\mathrm{e}^{-2 t_{m}}\right)}} \\
& \times \int p_{j-1, n}(y) \mathrm{e}^{-n V(y)-\frac{n \mathrm{e}^{-t_{m} y^{2}}}{2\left(1-\mathrm{e}^{\left.-2 t_{m}\right)}\right.}} \frac{\partial}{\partial y} \mathrm{e}^{-\frac{n x y}{2 \sinh t_{m}}} \mathrm{~d} y \\
= & 2 \sinh t_{m} \int\left(-p_{j-1, n}^{\prime}(y) / n\right. \\
& \left.+p_{j-1, n}(y) V^{\prime}(y)+p_{j-1, n}(y) \frac{\mathrm{e}^{-t_{m}} y}{2 \sinh t_{m}}\right) \\
& \times \mathrm{e}^{-n\left(V(y)+\frac{\mathrm{e}^{-2 t_{m}}\left(x^{2}+y^{2}\right)-2 \mathrm{e}^{-t_{m}} x y}{2\left(1-\mathrm{e}^{\left.-2 t_{m}\right)}\right.}\right)} \mathrm{d} y .
\end{aligned}
$$

The statement now follows by a rewriting of the latter using the recurrence matrix $\mathcal{J}$ and using (3.4).

From Lemma 3.8, it in particular follows that if the recurrence coefficients for the orthogonal polynomials have the required asymptotic behavior, then also the recurrence coefficients for $\phi_{j, m}$ have the required behavior and Theorems 2.3 and 2.7 apply.

PROPOSITION 3.9. If the recurrence coefficients $a_{k, n}$ and $b_{k, n}$ for $p_{k, n}$ satisfy

$$
a_{n+k, n} \rightarrow a, \quad b_{n+k, n} \rightarrow b,
$$

as $n \rightarrow \infty$, then Theorem 2.2 applies where

$$
a^{(m)}(z)=2 \sinh \left(t_{m}\right)\left(V^{\prime}(a z+b+a / z)\right)_{+}+\mathrm{e}^{-t_{m}}(a z+b+a / z),
$$


where $\left(V^{\prime}(a z+b+a / z)\right)_{+}$is the part of the Laurent polynomial $V^{\prime}(a z+b+a / z)$ containing the nonnegative powers.

Moreover, Theorem 2.6 also applies with

$$
\sum_{j} a_{j}(t) z^{j}=2 \sinh (t)\left(V^{\prime}(a z+b+a / z)\right)_{+}+\mathrm{e}^{-t}(a z+b+a / z) .
$$

The conditions of the latter proposition are met, when the polynomial $V$ is such that the zeros of $p_{n}$ accumulate on a single interval [17]. This happens, for example, when $V$ is convex.

Finally, we note that the above model is a special case of the Hermitian multimatrix model. In the present setting, the limiting distribution of points at a given time $t$ can also be computed using the recurrence coefficients, as was done in [20] for the special case where $V$ is an even quartic. Moreover, in [20] it was shown that this limiting distribution is a particular component of a vector of measures that uniquely minimizes an equilibrium problem.

3.3. Brownian bridges with starting and ending at several points. Next, we briefly comment on a generalization to the model of Brownian bridges from the Introduction. Instead of conditioning that all paths start and end at the same point, we allow for several starting points, say $x_{1}, \ldots, x_{r}$, and several ending points, $y_{1}, \ldots, y_{s}$ keeping $r, s \in \mathbb{N}$ fixed as $n \rightarrow \infty$. This more general model also fits within the framework of the present paper. Indeed, also in this case we also get polynomials that satisfy orthogonality relations, but these will now be multiple orthogonal of mixed type [16]. In the special case that $s=1$ or $r=1$, we obtain multiple Hermite polynomials [3]. Also for such polynomials recurrence relations exist and one may try to analyze the global fluctuations for such systems using the techniques of the present paper. However, preliminary calculations by the authors indicate that the recurrence matrices do no longer have Laurent structure at infinity, but we encounter block Laurent matrices instead. In the case of $r=1$ and $s=1$, the author has verified in preliminary calculations that the ideas in the present paper can be extended to prove Gaussian Free Field correlations in that case. This extension requires some nontrivial effort and will therefore be presented in a forthcoming manuscript. The general case of arbitrary $r$ and $s$ also falls within the framework but it seems that more technicalities need to be overcome. The author intends to return to this question in future work.

3.4. Lozenge tilings of a hexagon. The last example that we will treat is that of lozenge tilings of an $a b c$-hexagon. See Figures 5 and 6. This well-studied model can also be viewed as a model of discrete non-intersecting paths. It was proved in [43] (in a more general context) that the height function associated to the paths indeed has Gaussian Free Field fluctuations. We will show here that it also follows from our general results. We first give the two equivalent descriptions of the model in terms of tilings and in terms of non-intersecting paths, starting with the latter. 

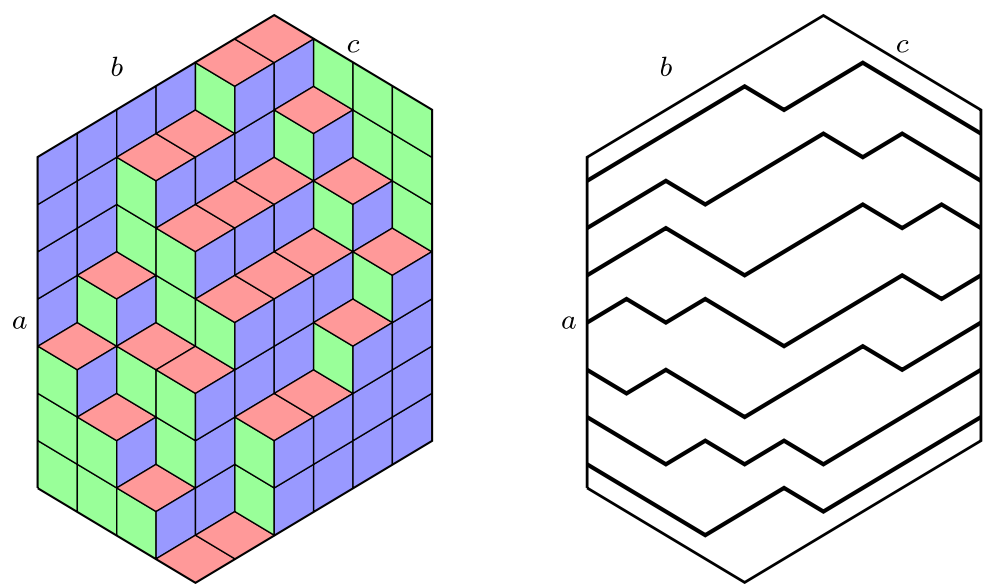

FIG. 5. A lozenge tiling of an abc-hexagon (l) and the equivalent representation in terms of non-intersecting paths $(r)$.

Fix $a, b, c \in \mathbb{N}$ and without loss of generality we assume that $b \leq c$. Then we consider a collection $\vec{\gamma}$ of $c$ zig-zag paths $\gamma_{j}:\{0,1, \ldots, b+c\} \rightarrow \mathbb{Z}$ for $j=1, \ldots, a$ such that

1. They start $\gamma(0)=2 j-1$ and end at $\gamma(b+c)=c-b+2 j-1$ for $j=$ $1, \ldots, a$.

2. At each step by one to the right, the path goes up or down by one, that is, $\gamma_{j}(k+1)=\gamma_{j}(k) \pm 1$.
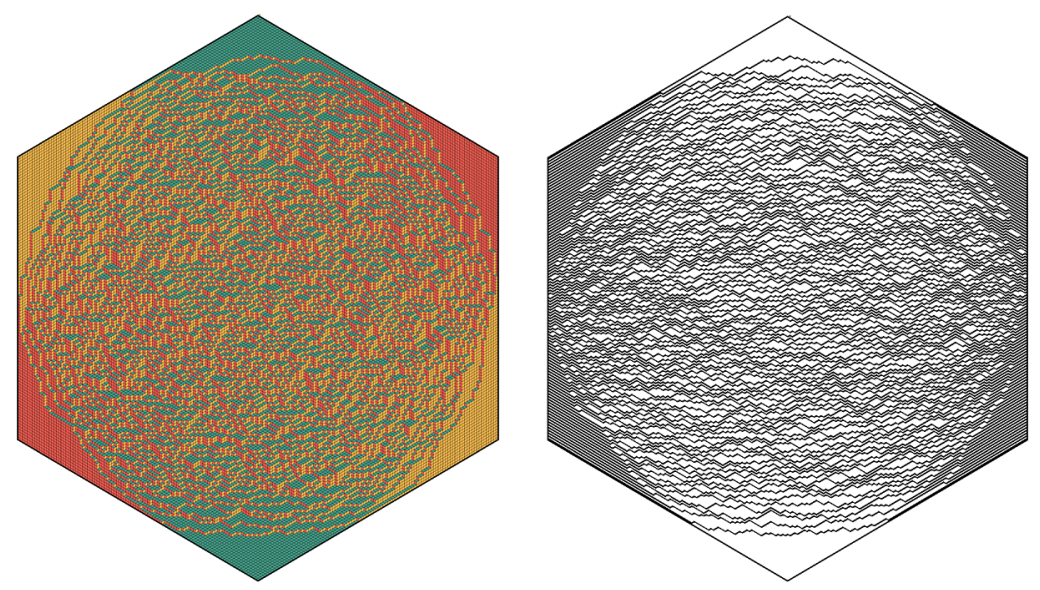

FIG. 6. A sampling of a random tiling of a large regular hexagon ( $l$ ) and the alternative representation in terms of non-intersecting paths $(r)$. The disordered regime, circle inside the hexagon, and the frozen corners are clearly visible. (These figures are produced using a code that was kindly provided to the author by Leonid Petrov.) 
3. The paths never cross $\gamma_{j}(k)<\gamma_{j+1}(k)$.

Note that due to the conditions on the starting and endpoints, each path will consist of $b$ down steps and $c$ up steps. We then take the uniform measure on all such $\vec{\gamma}$. This is equivalent to say that we consider $a$ random walkers with given starting and ending points conditioned never to intersect.

A different representation is that of lozenge tiling of the hexagon. Indeed, if we take an $a b c$-hexagon with corners $(0,0),(0,2 a),(c, 2 a+c),(b+c, 2 a+c-b)$, $(b+c, c-b)$ and $(b,-b)$ and tile this hexagon with lozenges of

$$
\text { type } \mathrm{I} \vee, \quad \text { type II } \downarrow \text { and type III } \diamond \text {. }
$$

To make the connection with the above path model, we associate to each tiling of the hexagon a collection of paths by drawing a down step on a lozenge of type I and an up step on a type III lozenge going through the centers of the lozenges. That is,

$$
\text { type I } \diamond, \quad \text { type II } \ominus \text { and type III } \diamond \text {. }
$$

It is then easy to see that this indeed defines a collection of zig-zag paths that do not intersect and start and end from the given points. Moreover, by taking the uniform measure on all possible tiling, we obtain the uniform measure on all zig-zag paths.

In $[25,30]$, it was proved that the locations of the paths $\left\{\left(k, \gamma_{j}(k)\right)\right\}_{k=1, j=1}^{b+c-1, a}$ form a determinantal point process with a kernel constructed out of the Hahn polynomials. We recall that the Hahn polynomials $q_{k, M}^{(\alpha, \beta)}$ are the orthonormal polynomials with respect to the weight

$$
w_{M}^{(\alpha, \beta)}(x)=\frac{1}{x !(x+\alpha) !(M+\beta-x) !(M-x) !},
$$

on $\{0,1, \ldots, M\}$, that is,

$$
\sum_{x=0}^{M} q_{k, M}^{(\alpha, \beta)}(x) q_{\ell, M}^{(\alpha, \beta)}(x) w_{N}^{(\alpha, \beta)}=\delta_{k \ell} .
$$

They have the explicit representation

$$
q_{k, M}^{(\alpha, \beta)}=\frac{(-M-\beta)_{k}(-M)_{k}}{k ! d_{k, M}^{(\alpha, \beta)}} F_{2}\left(\begin{array}{c}
-k, k-2 M-\alpha-\beta-1,-x \\
-M-\beta,-M
\end{array} ; 1\right),
$$

where ${ }_{3} F_{2}$ is the hypergeometric function and

$$
d_{k, M}^{(\alpha, \beta)^{2}}=\frac{(\alpha+\beta+M+1-k)_{M+1}}{(\alpha+\beta+2 k M+1-2 k) k !(\beta+M-k) !(\alpha+M-k) !(M-k) !},
$$

and $(\alpha)_{M}=\alpha(\alpha+1) \cdots(\alpha+M-1)$ denotes the usual Pochhammer symbol. From [33], Section 9.5, it follows that the normalized Hahn polynomials have the recurrence

$$
x q_{k, M}^{(\alpha, \beta)}(x)=a_{k+1} q_{k+1, M}^{(\alpha, \beta)}(x)+b_{k} q_{k, M}^{(\alpha, \beta)}(x)+a_{k} q_{k-1, M}^{(\alpha, \beta)}(x),
$$


where

$$
a_{k}=\sqrt{\frac{(M-k+1) k(M-k+1+\alpha)(M-k+1+\beta)(M-k+1+\alpha+\beta)(2 M-k+2+\alpha+\beta)}{(1+2 M-2 k+\alpha+\beta)(2+2 M-2 k+\alpha+\beta)^{2}(3+2 M-2 k+\alpha+\beta)}},
$$

and

$$
\begin{aligned}
b_{k}= & \frac{(2 M+\alpha+\beta+k-1)(M+\beta-k)(M-k)}{(2 M-2 k+\alpha+\beta)(2 M-2 k+\alpha+\beta+1)} \\
& +\frac{k(2 M+\alpha+\beta+1-k)(M-k+\alpha+1)}{(2 M-2 k+\alpha+\beta+2)(2 M-2 k+\alpha+\beta+1)} .
\end{aligned}
$$

Now we come back to the tiling process. We first need some notation in which we follow [30]. Set $\alpha_{r}=|c-r|, \beta_{r}=|b-r|, L_{r}=b-b_{r}$ and

$$
M_{r}= \begin{cases}r+a-1, & 0 \leq r \leq b, \\ b+a-1, & b \leq r \leq c, \\ a+b+c-1-r, & c \leq r \leq b+c .\end{cases}
$$

Then, as shown in $[25,30]$, the locations of the paths $\left\{\left(m, \gamma_{j}(m)\right\}\right.$ (or, equivalently, the centers of the tiles of type II) form a determinantal point process on $\{0,1, \ldots, B+C\} \times \mathbb{Z}$ with kernel

$$
\begin{aligned}
K_{a}\left(r, L_{r}+2 x+1, s, L_{s}+2 y+1\right) & \\
= & \begin{cases}\sum_{k=0}^{a-1} \sqrt{\frac{(a+s-1-k) !(a+b+c-r-1-k) !}{(a+r-1-k) !(a+b+c-s-1-k) !}} q_{k, M_{r},}^{\left(\alpha_{r}, \beta_{r}\right)}(x) q_{k, M_{s}}^{\left(\alpha_{s}, \beta_{s}\right)}(y), & r \leq s, \\
-\sum_{k=a}^{\infty} \sqrt{\frac{(a+s-1-k) !(a+b+c-r-1-k) !}{(a+r-1-k) !(a+b+c-s-1-k) !}} q_{k, M_{r}}^{\left(\alpha_{r}, \beta_{r}\right)}(x) q_{k, M_{s}}^{\left(\alpha_{s}, \beta_{s}\right)}(y), & r>s .\end{cases}
\end{aligned}
$$

The question of interest is what happens with the system as the hexagon becomes large. That is, we introduce a big parameter $n$ and scale $a, b$ and $c$ such that

$$
a / n \rightarrow 1, \quad b / n \rightarrow B>0, \quad c / n \rightarrow C>0 .
$$

Then we take $\rho_{m} \in(0, B+C)$ for $m=1, \ldots, N$ and set $r_{r}=\left[n \rho_{r}\right]$ where [-] denotes the integer part. We also rescale the process along the vertical axis by $n$ (hence we replace $x$ by $[n x]$ ).

We then set

$$
p_{j, m}(x)=q_{j, M_{r_{m}}}^{\alpha_{r_{m}}, \beta_{r_{m}}}([n x])
$$

and

$$
c_{j, m}=\sqrt{\frac{\left(a+b+c-r_{m}-1-j\right) !}{\left(a+r_{m}-1-j\right) !}},
$$

and consider the probability measure (2.1) with $\phi_{j, m}=c_{j, m} p_{j-1, m}$ and $\psi_{j, m}=$ $1 / c_{j, m} p_{j-1, m}$ as in (2.10) [with $T_{m}(x, y)=\sum_{j=0}^{\infty} c_{j, m+1} / c_{j, m} p_{j, m}(x) p_{j, m+1}(y)$ ]. 
Denote the recurrence coefficients for $p_{j, m}$ by $a_{j, m}^{(n)}$ and $b_{j, m}^{(n)}$. Then, from (3.8), (3.9) and the choice of the parameters is not hard to show that there exists functions $F_{1}$ and $F_{2}$ as $n \rightarrow \infty$,

$$
a_{n+k, m}^{(n)} \rightarrow F_{1}\left(\rho_{m} ; B, C\right), \quad b_{n+k, m}^{(n)} \rightarrow F_{2}\left(\rho_{m} ; B, C\right),
$$

for any $k \in \mathbb{N}$. In other words, condition (2.18) is satisfied. Moreover, we also we easily verify (2.19) and find

$$
\lim _{n \rightarrow \infty} \frac{c_{l, m}}{c_{k, m}}=\mathrm{e}^{\tau_{m}(k-l)} \quad \text { with } \tau_{m}=\frac{1}{2} \ln \frac{1+B+C-\rho_{m}}{1+\rho_{m}} .
$$

Hence we see that Theorem 2.4 applies. Also note that after rescaling with $n$ the hexagon will always be contained in a fixed compact set for every $n$, hence also Corollary 2.5 applies.

Similarly, in the same way one can verify that Corollary 2.8, Theorem 2.9 and Theorem 2.10 apply. We leave the precise statement to the reader.

4. From linear statistics to the recurrence matrices. In this section, we show how the moments and cumulants of the linear statistics are connected to the matrices $\mathbb{J}_{m}$.

The determinantal structure of the process means that we can express the moments of linear statistics in terms of the kernel $K_{n, N}(2.8)$. Indeed, it is standard that from (2.7) and some computations one can show

$$
\mathbb{E} X_{n}(f)=\sum_{m=1}^{N} \int f(m, x) K_{n, N}(m, x, m, x) \mathrm{d} \mu_{m}(x),
$$

implying that $K_{n, N}(m, x, m, x)$ is the mean density. Moreover,

$$
\begin{aligned}
\operatorname{Var} X_{n}(f)= & \sum_{m=1}^{N} \int f(m, x)^{2} K_{n, N}(m, x, m, x) \mathrm{d} \mu_{m}(x) \\
& -\sum_{m_{1}, m_{2}=1}^{N} \iint f\left(m_{1}, x_{1}\right) f\left(m_{2}, x_{2}\right) K_{n, N}\left(x_{1}, m_{1}, x_{2}, m_{2}\right) \\
& \times K_{n, N}\left(x_{2}, m_{2}, x_{1}, m_{1}\right) \mathrm{d} \mu_{m_{1}}\left(x_{1}\right) \mathrm{d} \mu_{m_{2}}\left(x_{2}\right)
\end{aligned}
$$

and similar expressions hold for the higher terms.

Although we use these expressions in the proofs of Theorem 2.5 and 2.9, the general strategy in this paper is based on a different approach. The key identity is the following lemma, connecting the moments to the recurrence matrices $\mathbb{J}_{m}$.

LEMMA 4.1. Suppose that $f(m, x)$ is a polynomial in $x$ and $\mathbb{J}_{m}$ is bounded for $m=1, \ldots, N$, then

$$
\mathbb{E}\left[\mathrm{e}^{\lambda X_{n}(f)}\right]=\operatorname{det}\left(\left(\mathrm{e}^{\lambda f\left(1, \mathbb{J}_{1}\right)} \mathrm{e}^{\lambda f\left(2, \mathbb{J}_{2}\right)} \cdots \mathrm{e}^{\lambda f\left(N, \mathbb{J}_{N}\right)}\right)_{i, j}\right)_{i, j=1}^{n} \cdot
$$


In case one of the $\mathbb{J}_{m}$ is an unbounded matrix, the equality is understood as an equality between formal power series by expanding each exponential. More precisely, with $R_{M}(x)=\sum_{k=0}^{M} x^{k} / k$ ! consider the expansion

$$
\begin{aligned}
& \operatorname{det}\left(\left(R_{M}\left(\lambda f\left(1, \mathbb{J}_{1}\right)\right) R_{M}\left(\lambda f\left(2, \mathbb{J}_{2}\right)\right) \cdots R_{M}\left(\lambda f\left(N, \mathbb{J}_{N}\right)\right)\right)_{i, j}\right)_{i, j=1}^{n} \\
& =\sum_{k=0}^{\infty} D_{k, M}(f) \frac{\lambda^{k}}{k !}
\end{aligned}
$$

then we have $\mathbb{E}\left[\left(X_{n}(f)\right)^{k}\right]=D_{k, M}(f)$, for $k \leq M$.

REMARK 4.1. Before we come to the proof, we note that since the $\mathbb{J}_{m}$ 's are banded matrices and each $f_{m}(m, x)$ is a polynomial in $x$, we have that $f\left(m, \mathbb{J}_{m}\right)$ and the product of the matrices $R_{m}\left(f\left(\mathbb{J}_{m}, m\right)\right)$ are well-defined so that the determinant at the left-hand side of (4.3) makes sense.

PROOF. It is enough to prove (4.3). In case all $\mathbb{J}_{m}$ are bounded, we then obtain (4.2) by taking the limit $M \rightarrow \infty$ in a straightforward way.

We first note that the first $M+1$ terms of the (formal) expansions

$$
\mathbb{E}\left[\mathrm{e}^{\lambda X_{n}(f)}\right]=\mathbb{E}\left[\prod_{j, m=1}^{n, N} \mathrm{e}^{\lambda f\left(m, x_{m, j}\right)}\right]
$$

and

$$
\mathbb{E}\left[\prod_{j, m=1}^{n, N} R_{M}\left(\lambda f\left(m, x_{m, j}\right)\right)\right]
$$

are equal. To prove the lemma, it thus suffices to prove that the last expectation equals the left-hand side of (4.3). To this end, we note

$$
\begin{aligned}
\mathbb{E}\left[\prod_{j, m=1}^{n, N} R_{M}\left(\lambda f\left(m, x_{m, j}\right)\right)\right] \\
=\frac{1}{(n !)^{N}} \int \cdots \int\left(\prod_{j, m=1}^{n, N} R_{M}\left(\lambda f\left(m, x_{m, j}\right)\right)\right) \operatorname{det}\left(\phi_{j, 1}\left(x_{1, k}\right)\right)_{j, k=1}^{n} \\
\quad \times \prod_{m=1}^{N-1} \operatorname{det}\left(T_{m}\left(x_{m, i}, x_{m+1, j}\right)\right)_{i, j=1}^{n} \\
\quad \times \operatorname{det}\left(\psi_{j, N}\left(x_{N, k}\right)\right)_{j, k=1}^{n} \prod_{m=1}^{N} \prod_{j=1}^{n} \mathrm{~d} \mu_{m}\left(x_{m, j}\right) \\
=\frac{1}{(n !)^{N}} \int \cdots \int \operatorname{det}\left(\phi_{j, 1}\left(x_{1, k}\right)\right)_{j, k=1}^{n}
\end{aligned}
$$




$$
\begin{aligned}
& \times \prod_{m=1}^{N-1} \operatorname{det}\left(R_{M}\left(\lambda f\left(m, x_{m, i}\right)\right) T_{m}\left(x_{m, i}, x_{m+1, j}\right)\right)_{i, j=1}^{n} \\
& \times \operatorname{det}\left(R_{M}\left(\lambda f\left(N, x_{N, k}\right)\right) \psi_{j, N}\left(x_{N, k}\right)\right)_{j, k=1}^{n} \prod_{m=1}^{N} \prod_{j=1}^{n} \mathrm{~d} \mu_{m}\left(x_{m, j}\right) .
\end{aligned}
$$

For convenience, we set some notation $A_{m}=R_{M}\left(\lambda f\left(m, \mathbb{J}_{m}\right)\right)$. Now the statement is a special case (where $s_{j}=j$ ) of the more general claim

$$
\begin{aligned}
& \frac{1}{(n !)^{N}} \int \cdots \int \operatorname{det}\left(\phi_{s_{j}, 1}\left(x_{1, k}\right)\right)_{j, k=1}^{n} \\
& \quad \times \prod_{m=1}^{N-1} \operatorname{det}\left(R_{M}\left(\lambda f\left(m, x_{m, i}\right)\right) T_{m}\left(x_{m, i}, x_{m+1, j}\right)\right)_{i, j=1}^{n} \\
& \quad \times \operatorname{det}\left(R_{M}\left(\lambda f\left(N, x_{N, k}\right)\right) \psi_{j, N}\left(x_{N, k}\right)\right)_{j, k=1}^{n} \prod_{m=1}^{N} \prod_{j=1}^{n} \mathrm{~d} \mu_{m}\left(x_{m, j}\right) \\
& \quad=\operatorname{det}\left(\left(A_{1} \cdots A_{N}\right)_{s_{i}, s_{j}}\right)_{i, j=1}^{n},
\end{aligned}
$$

for any $s_{1}<\cdots<s_{n}$.

The proof of (4.4) goes by induction to $N$.

The case of $N=1$ is a direct consequence of Andreiéf's identity in (2.3)

$$
\begin{aligned}
& \frac{1}{n !} \int \cdots \int \operatorname{det}\left(\phi_{s_{j}, 1}\left(x_{1, k}\right)\right)_{j, k=1}^{n} \\
& \quad \times \operatorname{det}\left(R_{M}\left(\lambda f\left(1, x_{1, k}\right)\right) \psi_{s_{j}, 1}\left(x_{1, k}\right)\right)_{j, k=1}^{n} \prod_{j=1}^{n} \mathrm{~d} \mu_{1}\left(x_{1, j}\right) \\
& \quad=\operatorname{det}\left(\int R_{M}(\lambda f(1, x)) \phi_{s_{j}, 1}(x) \psi_{s_{i}, 1}(x) \mathrm{d} \mu_{1}(x)\right)_{i, j=1}^{n} \\
& \quad=\operatorname{det}\left(\left(A_{1}\right)_{s_{i}, s_{j}}\right)_{i, j=1}^{n} .
\end{aligned}
$$

For $N>1$, we use Andreiéf's identity to write

$$
\begin{aligned}
& \frac{1}{n !} \int \cdots \int \operatorname{det}\left(\phi_{s_{j}, 1}\left(x_{1, k}\right)\right)_{j, k=1}^{n} \\
& \quad \times \operatorname{det}\left(R_{M}\left(\lambda f\left(x_{1, i}, 1\right)\right) T_{1}\left(x_{1, i}, x_{2, j}\right)\right)_{i, j=1}^{n} \prod_{j=1}^{n} \mathrm{~d} \mu_{1}\left(x_{1, j}\right) \\
& \quad=\operatorname{det}\left(\int T_{1}\left(x, x_{2, i}\right) R_{M}(\lambda f(x, 1)) \phi_{s_{j}, N}(x) \mathrm{d} \mu_{1}(x)\right) .
\end{aligned}
$$


By using the recurrence and the fact that

$$
\int T_{1}\left(x, x_{2, i}\right) \phi_{k, 1}(x) \mathrm{d} \mu_{1}(x)=\phi_{k, 2}\left(x_{2, i}\right)
$$

we find that the right-hand side of (4.5) can be written as

$$
\begin{aligned}
& \operatorname{det}\left(\sum_{k} A_{s_{j}, k} \phi_{k, 2}\left(x_{2, i}\right)\right)_{i, j=1}^{n} \\
& \quad=\sum_{l_{1}<l_{2}<\cdots<l_{n}} \operatorname{det}\left(A_{s_{j}, l_{i}}\right)_{i, j=1}^{n} \operatorname{det}\left(\phi_{l_{j}, 2}\left(x_{2, i}\right)\right)_{i, j=1}^{n}
\end{aligned}
$$

where we used Cauchy-Binet in the last step. By inserting the latter with (4.5) back into the left-hand side of (4.4) and using the induction hypothesis we find that the left-hand side of (4.4) can be written as

$$
\begin{aligned}
& \sum_{l_{1}<l_{2}<\cdots<l_{n}} \operatorname{det}\left(\left(A_{1}\right)_{s_{k}, l_{j}}\right)_{j, k=1}^{n} \operatorname{det}\left(\left(A_{2} \ldots A_{N}\right)_{l_{j}, s_{i}}\right)_{i, j=1}^{n} \\
& \quad=\operatorname{det}\left(\left(A_{1} \ldots A_{N}\right)_{s_{i}, s_{k}}\right)_{i, k=1}^{n},
\end{aligned}
$$

where we used Cauchy-Binet in the last step again. This proves the claim in (4.4), and hence the statement.

This lemma also has a convenient consequence. Since all $\mathbb{J}_{m}$ are banded, each entry

$$
\left(R_{M}\left(\lambda f\left(1, \mathbb{J}_{1}\right)\right) R_{M}\left(\lambda f\left(2, \mathbb{J}_{2}\right)\right) \cdots R_{M}\left(\lambda f\left(N, \mathbb{J}_{N}\right)\right)\right)_{i, j}
$$

for $i, j=1, \ldots, n$ only depends on some entries of the individual $\mathbb{J}_{m}$ 's. By writing out the matrix product it is not hard to see that these entries do not depend on any $\left(\mathbb{J}_{m}\right)_{r s}$ for $m=1, \ldots, N$ and $r, s>S$ for some sufficiently large $S$. Hence if we define the cut-offs

$$
\left(\mathbb{J}_{m, S}\right)_{j, k}= \begin{cases}\left(\mathbb{J}_{m}\right)_{j, k}, & j, k \leq S \\ 0, & \text { otherwise }\end{cases}
$$

and expand

$$
\begin{aligned}
\operatorname{det}\left(\left(\mathrm{e}^{\lambda f\left(1, \mathbb{J}_{m, S}\right)} \mathrm{e}^{\lambda f\left(2, \mathbb{J}_{2, S}\right)} \cdots \mathrm{e}^{\lambda f\left(N, \mathbb{J}_{N, S}\right)}\right)_{i, j}\right)_{i, j=1}^{n} \\
=\sum_{k=0}^{\infty} \frac{\lambda^{k}}{k !} \tilde{\mathcal{D}}_{k, S}(f),
\end{aligned}
$$

then for each $k \in \mathbb{N}$ we have $\mathbb{E}\left[\left(X_{n}(f)\right)^{k}\right]=\tilde{\mathcal{D}}_{k, S}(f)$ for sufficient large $S$ (which may depend on $k$ ). The benefit is that the matrix in the determinant consists of a product of bounded operators and the series is convergent. Hence we do not have to worry about formal series and this will be convenient for technical reasons. 
Instead of the moments, it will be more convenient to work with the cumulants $\mathcal{C}_{k}\left(X_{n}(f)\right)$. These are special combinations of the linear statistic, determined by the (formal) generating function

$$
\log \mathbb{E}\left[\exp \left(\lambda X_{n}(f)\right)\right]=\sum_{k=1}^{\infty} \frac{\lambda^{k}}{k !} \mathcal{C}_{k}\left(X_{n}(f)\right) .
$$

Note that $\mathcal{C}_{1}\left(X_{n}(f)\right)=\mathbb{E} X_{n}(f)$ and $\mathcal{C}_{2}\left(X_{n}(f)\right)=\operatorname{Var} X(f)$. The $k$ th cumulant can be expressed in terms of the first $k$ moments and vice versa. Since the first terms in the expansion on the right-hand side of (4.6) are the moments, we can take the logarithm at both sides and immediately obtain the following lemma.

\section{LEMMA 4.2. Let $\mathcal{C}_{k, S}(f)$ be the coefficients in the series}

$$
\log \operatorname{det}\left(\left(\mathrm{e}^{\lambda f\left(1, \mathbb{J}_{m, S}\right)} \mathrm{e}^{\lambda f\left(2, \mathbb{J}_{2, S}\right)} \cdots \mathrm{e}^{\lambda f\left(N, \mathbb{J}_{N, S}\right)}\right)_{i, j}\right)_{i, j=1}^{n}=\sum_{k=0}^{\infty} \frac{\lambda^{k}}{k !} \mathcal{C}_{k, S}(f),
$$

then $\mathcal{C}_{k}\left(X_{n}(f)\right)=\mathcal{C}_{k, S}(f)$ for sufficiently large $S$.

Using this representation, we will give useful expressions for all the cumulants. We will do this in the next section in a more general setup.

5. Expansions of Fredholm determinant. In this section, we will look at the expansion given in Lemma 4.2, where we replace the $f\left(m, \mathbb{J}_{m, S}\right)$ 's in the determinant by general banded and bounded operators $A_{m}$.

5.1. Preliminaries. We start by recalling traces and determinants for trace class operators. We refer to [24, 46] for more details.

For a compact operator $A$ on a separable Hilbert space $\mathcal{H}$, we define the singular values

$$
\sigma_{1}(A) \geq \sigma_{2}(A) \geq \sigma_{3}(A) \geq \cdots>0,
$$

as the square roots of the eigenvalues of the self-adjoint compact operators $A^{*} A$. The space of trace class operators is then defined as the Banach space

$$
\mathcal{B}_{1}(\mathcal{H})=\left\{A \mid \sum_{k=1}^{\infty} \sigma_{k}(A)<\infty\right\}
$$

equipped with the trace norm

$$
\|A\|_{1}=\sum_{k=1}^{\infty} \sigma_{k}(A)
$$

The space of Hilbert-Schmidt operators is then defined as the Hilbert space

$$
\mathcal{B}_{2}(\mathcal{H})=\left\{A \mid \sum_{k=1}^{\infty} \sigma_{k}(A)^{2}<\infty\right\}
$$


equipped with the Hilbert-Schmidt norm

$$
\|A\|_{2}=\left(\sum_{k=1}^{\infty} \sigma_{k}(A)^{2}\right)^{1 / 2} .
$$

We also denote the operator norm by $\|A\|_{\infty}$ and the space of bounded operators by $\mathcal{B}_{\infty}(\mathcal{H})$.

The following identities are standard. For any $A \in \mathcal{B}_{\infty}(\mathcal{H})$ and $B \in \mathcal{B}_{1}(\mathcal{H})$, we have

$$
\|A B\|_{1}, \quad\|B A\|_{1} \leq\|A\|_{\infty}\|B\|_{1} .
$$

Similarly, for any $A \in \mathcal{B}_{\infty}(\mathcal{H})$ and $B \in \mathcal{B}_{2}(\mathcal{H})$ we have

$$
\|A B\|_{2}, \quad\|B A\|_{2} \leq\|A\|_{\infty}\|B\|_{2} .
$$

For any $A, B \in \mathcal{B}_{2}(\mathcal{H})$, we have

$$
\|A B\|_{1} \leq\|A\|_{2}\|B\|_{2} .
$$

The trace class operators $\mathcal{B}_{1}(\mathcal{H})$ are precisely the operators for which we can define the trace, denote by $\operatorname{Tr} A$, by naturally extending the trace for finite rank operators. We note that

$$
|\operatorname{Tr} A|=\|A\|_{1} .
$$

For any trace class operator $A \in \mathcal{B}_{1}(\mathcal{H})$, we can also define the operator determinant $\operatorname{det}(I+A)$ by natural extension from the finite rank operators. Here, we note that

$$
\begin{aligned}
& |\operatorname{det}(I+A)-\operatorname{det}(I+B)| \\
& \quad \leq\|A-B\|_{1} \exp \left(\|A\|_{1}+\|B\|_{1}+1\right) .
\end{aligned}
$$

A particular relation between the trace and the determinant that we will use is the following

$$
\log \operatorname{det}(I+A)=\sum_{j=1}^{\infty} \frac{(-1)^{j+1}}{j} \operatorname{Tr} A^{j},
$$

valid for any $A \in \mathcal{B}_{1}(\mathcal{H})$ for which $\|A\|_{\infty}<1$ (ensuring the convergence of the right-hand side).

5.2. A cumulant-type expansion. Let $A_{1}, \ldots, A_{N}$ be bounded operator on $\ell_{2}(\mathbb{N})$ [in the coming analysis we will identify bounded operators on $\ell_{2}(\mathbb{N})$ with their semi-infinite matrix representations with respect to the canonical basis]. We will also use the notation $P_{n}$ for the projection operators on $\ell_{2}(\mathbb{N})$ defined by

$$
P_{n}:\left(x_{1}, x_{2}, \ldots\right) \mapsto\left(x_{1}, \ldots, x_{n}, 0,0, \ldots\right),
$$


and $Q_{n}=I-P_{n}$. Then

$$
\operatorname{det}\left(I+P_{n}\left(\mathrm{e}^{\lambda A_{1}} \mathrm{e}^{\lambda A_{2}} \cdots \mathrm{e}^{\lambda A_{N}}-I\right) P_{n}\right)
$$

is a well-defined and entire function of $\lambda$. By taking $A=\mathrm{e}^{\lambda A_{1}} \mathrm{e}^{\lambda A_{2}} \cdots \mathrm{e}^{\lambda A_{N}}-I$ in (5.1) for sufficiently small $\lambda$, we define $C_{k}^{(n)}\left(A_{1}, \ldots, A_{N}\right)$ by

$$
\begin{gathered}
\log \operatorname{det}\left(I+P_{n}\left(\mathrm{e}^{\lambda A_{1}} \mathrm{e}^{\lambda A_{2}} \cdots \mathrm{e}^{\lambda A_{N}}-I\right) P_{n}\right) \\
=\sum_{k=1}^{\infty} \lambda^{k} C_{k}^{(n)}\left(A_{1}, \ldots, A_{N}\right),
\end{gathered}
$$

which is valid for small $\lambda$. In Lemma 4.2 we have shown that the relation between the cumulant $\mathcal{C}_{k}\left(X_{n}(f)\right)$ and the general coefficient $C_{k}^{(n)}\left(A_{1}, \ldots, A_{N}\right)$ is given by

$$
\mathcal{C}_{k}\left(X_{n}(f)\right)=C_{k}^{(n)}\left(f\left(1, \mathbb{J}_{1, S}\right), \ldots, f\left(N, \mathbb{J}_{N, S}\right)\right),
$$

for sufficiently large $S$. We will use this connection only in Section 6 when we give the proofs of the main results. In this section, we focus on general properties of $C_{k}^{(n)}\left(A_{1}, \ldots, A_{N}\right)$. To start with, an easy consequence of the above is the following.

LEMMA 5.1. We have

$$
\begin{aligned}
C_{k}^{(n)}( & \left.A_{1}, \ldots, A_{N}\right) \\
& =\frac{1}{2 \pi \mathrm{i}} \oint_{|z|=\rho} \operatorname{Tr} \log \left(I+P_{n}\left(\mathrm{e}^{\lambda A_{1}} \mathrm{e}^{\lambda A_{2}} \cdots \mathrm{e}^{\lambda A_{N}}-I\right) P_{n}\right) \frac{\mathrm{d} \lambda}{\lambda^{k+1}},
\end{aligned}
$$

where $0<\rho<\left(2 \sum_{j=1}^{N}\left\|A_{j}\right\|_{\infty}\right)^{-1}$.

PROOF. The only remaining is the choice of $\rho$. To this end, we note that

$$
\begin{aligned}
& \left\|P_{n}\left(\mathrm{e}^{\lambda A_{1}} \mathrm{e}^{\lambda A_{2}} \cdots \mathrm{e}^{\lambda A_{N}}-I\right) P_{n}\right\|_{\infty} \\
& \quad \leq|\lambda| \sum_{j=1}^{N}\left\|A_{j}\right\|_{\infty} \exp \left(|\lambda| \sum_{j=1}^{N}\left\|A_{j}\right\|_{\infty}\right),
\end{aligned}
$$

and that $\frac{1}{2} \mathrm{e}^{\frac{1}{2}}<1$. Hence the integrand at the right-hand side of (5.4) is well-defined and analytic for $|\lambda|<\rho$. This proves the statement.

By expanding the logarithm, we obtain another useful expression. 
LEMMA 5.2. We have

$$
\begin{aligned}
C_{k}^{(n)}\left(A_{1}, \ldots, A_{N}\right) & \\
= & \sum_{j=1}^{k} \frac{(-1)^{j+1}}{j} \sum_{\substack{\ell_{1}+\cdots+\ell_{j}=k \\
\ell_{i} \geq 1}} \\
& \sum_{\left(r_{s, v}\right) \in R_{\ell_{1}, \ldots, \ell_{j}}} \frac{\operatorname{Tr} \prod_{s=1}^{j}\left(P_{n} A_{1}^{r_{s, 1}} \cdots A_{N}^{r_{s, N}} P_{n}\right)}{r_{1,1} ! \cdots r_{1, N} ! r_{2,1} ! \cdots r_{2, N} ! \cdots r_{j, 1} ! \cdots r_{j, N} !},
\end{aligned}
$$

where

$$
R_{\ell_{1}, \ldots, \ell_{j}}=\left\{\left(r_{s, v}\right)_{s=1, v=1}^{j, N} \mid r_{s, v} \in\{0,1,2, \ldots\}, \sum_{v=1}^{N} r_{s, v}=\ell_{s}\right\}
$$

ProOF. We note the following expansion, which is valid for any bounded operators $A_{1}, \ldots, A_{N}$ and sufficiently small $\lambda$,

$$
\begin{aligned}
& \log \left(1+P_{n}\left(\mathrm{e}^{\lambda A_{1}} \mathrm{e}^{\lambda A_{2}} \cdots \mathrm{e}^{\lambda A_{N}}-I\right) P_{n}\right) \\
& =\sum_{j=1}^{\infty} \frac{(-1)^{j+1}}{j} \operatorname{Tr}\left(P_{n}\left(\mathrm{e}^{\lambda A_{1}} \mathrm{e}^{\lambda A_{2}} \cdots \mathrm{e}^{\lambda A_{N}}-I\right) P_{n}\right)^{j} \\
& =\sum_{j=1}^{\infty} \frac{(-1)^{j+1}}{j}\left(\sum_{r_{1}, \ldots, r_{N}=0}^{\infty} \lambda^{r_{1}+\cdots+r_{N}} \operatorname{Tr} P_{n} A_{1}^{r_{1}} \cdots A_{N}^{r_{N}} P_{n}\right)^{j} \\
& =\sum_{j=1}^{\infty} \frac{(-1)^{j+1}}{j}\left(\sum_{\ell=1}^{\infty} \lambda^{\ell} \sum_{r_{1}, \ldots, r_{N}=\ell} \frac{\operatorname{Tr} P_{n} A_{1}^{r_{1}} \cdots A_{N}^{r_{N}} P_{n}}{r_{1} ! \cdots r_{N} !}\right)^{j} \\
& =\sum_{j=1}^{\infty} \frac{(-1)^{j+1}}{j} \sum_{\ell_{1}, \ldots, \ell_{j}=1}^{\infty} \lambda^{\ell_{1}+\cdots+\ell_{j}} \\
& \times \sum_{\left(r_{s, v}\right) \in R_{\ell_{1}, \ldots, \ell_{j}}} \frac{\operatorname{Tr} \prod_{s=1}^{j}\left(P_{n} A_{1}^{r_{s, 1}} \cdots A_{n}^{r_{s, N}} P_{n}\right)}{r_{1,1} ! \cdots r_{1, N} ! r_{2,1} ! \cdots r_{2, N} ! \cdots r_{j, 1} ! \cdots r_{j, N} !} \\
& =\sum_{j=1}^{\infty} \frac{(-1)^{j+1}}{j} \sum_{k=j}^{\infty} \lambda^{k} \\
& \times \sum_{\ell_{1}+\cdots+\ell_{j}=k} \sum_{\left(r_{s, v}\right) \in R_{\ell_{1}, \ldots, \ell_{j}}} \frac{\operatorname{Tr} \prod_{s=1}^{j}\left(P_{n} A_{1}^{r_{s, 1}} \cdots A_{n}^{r_{s, N}} P_{n}\right)}{r_{1,1} ! \cdots r_{1, N} ! r_{2,1} ! \cdots r_{2, N} ! \cdots r_{j, 1} ! \cdots r_{j, N} !}
\end{aligned}
$$




$$
\begin{aligned}
= & \sum_{k=1}^{\infty} \lambda^{k} \sum_{j=1}^{k} \frac{(-1)^{j+1}}{j} \sum_{\ell_{1}+\cdots+\ell_{j}=k} \\
& \quad \sum_{\left(r_{s, v}\right) \in R_{\ell_{1}, \ldots, \ell_{j}}} \frac{\operatorname{Tr} \prod_{s=1}^{j}\left(P_{n} A_{1}^{r_{s, 1}} \cdots A_{n}^{r_{s, N}} P_{n}\right)}{r_{1,1} ! \cdots r_{1, N} ! r_{2,1} ! \cdots r_{2, N} ! \cdots r_{j, 1} ! \cdots r_{j, N} !} .
\end{aligned}
$$

This proves the statement.

In the proofs of the main theorems, it will be important to have the following continuity result.

Lemma 5.3. Let $A_{1}, \ldots, A_{N}$ and $B_{1}, \ldots, B_{N}$ be semi-infinite matrices, then

$$
\begin{aligned}
& \left|C_{k}^{(n)}\left(A_{1}, \ldots, A_{N}\right)-C_{k}^{(n)}\left(B_{1}, \ldots, B_{N}\right)\right| \\
& \quad \leq \frac{2 \mathrm{e}}{(2-\sqrt{\mathrm{e}})^{2}}\left(2 \max \left(\sum_{j=1}^{N}\left\|A_{j}\right\|_{\infty}, \sum_{j=1}^{N}\left\|B_{j}\right\|_{\infty}\right)\right)^{k-1} \sum_{j=1}^{N}\left\|A_{j}-B_{j}\right\|_{1} .
\end{aligned}
$$

PROOF. We start by writing

$$
\begin{aligned}
C_{k}^{(n)}( & \left.A_{1}, \ldots, A_{N}\right) \\
& -C_{k}^{(n)}\left(B_{1}, \ldots, B_{N}\right) \\
& \times \frac{1}{2 \pi \mathrm{i}} \oint_{|z|=\rho} \operatorname{Tr}\left(\log \left(I+P_{n}\left(\mathrm{e}^{\lambda A_{1}} \mathrm{e}^{\lambda A_{2}} \cdots \mathrm{e}^{\lambda A_{N}}-I\right) P_{n}\right)\right. \\
& \left.-\log \left(I+P_{n}\left(\mathrm{e}^{\lambda B_{1}} \mathrm{e}^{\lambda B_{2}} \cdots \mathrm{e}^{\lambda B_{N}}-I\right) P_{n}\right)\right) \frac{\mathrm{d} \lambda}{\lambda^{k+1}} .
\end{aligned}
$$

We estimate the integrand using

$$
|\operatorname{Tr}(\log (I+A)-\log (1+B))| \leq \frac{\|A-B\|_{1}}{\left(1-\|A\|_{\infty}\right)\left(1-\|B\|_{\infty}\right)}
$$

If we take $\rho=\left(2 \max \left(\sum_{j=1}^{N}\left\|A_{j}\right\|_{\infty}, \sum_{j=1}^{N}\left\|B_{j}\right\|_{\infty}\right)\right)^{-1}$, then

$$
\begin{gathered}
\left\|\left(\mathrm{e}^{\lambda A_{1}} \mathrm{e}^{\lambda A_{2}} \cdots \mathrm{e}^{\lambda A_{N}}-I\right)\right\|_{\infty} \leq \frac{\sqrt{\mathrm{e}}}{2}, \\
\left\|\left(\mathrm{e}^{\lambda B_{1}} \mathrm{e}^{\lambda B_{2}} \cdots \mathrm{e}^{\lambda B_{N}}-I\right)\right\|_{\infty} \leq \frac{\sqrt{\mathrm{e}}}{2}
\end{gathered}
$$


for $\lambda=\rho$. Moreover,

$$
\begin{aligned}
&\left\|\left(\mathrm{e}^{\lambda A_{1}} \mathrm{e}^{\lambda A_{2}} \cdots \mathrm{e}^{\lambda A_{N}}-I\right)-\left(\mathrm{e}^{\lambda B_{1}} \mathrm{e}^{\lambda B_{2}} \cdots \mathrm{e}^{\lambda B_{N}}-I\right)\right\|_{1} \\
&=\left\|\sum_{j=1}^{N} \mathrm{e}^{\lambda A_{1}} \cdots \mathrm{e}^{\lambda A_{j-1}}\left(\mathrm{e}^{\lambda A_{j}}-\mathrm{e}^{\lambda B_{j}}\right) \mathrm{e}^{\lambda B_{j+1}} \cdots \mathrm{e}^{\lambda B_{N}}\right\|_{1} \\
& \leq \sum_{j=1}^{N}\left\|\mathrm{e}^{\lambda A_{1}}\right\|_{\infty} \\
& \quad \cdots\left\|\mathrm{e}^{\lambda A_{j-1}}\right\|_{\infty}\left\|\mathrm{e}^{\lambda A_{j}}-\mathrm{e}^{\lambda B_{j}}\right\|_{1}\left\|\mathrm{e}^{\lambda B_{j+1}}\right\|_{\infty} \cdots\left\|\mathrm{e}^{\lambda B_{N}}\right\|_{\infty} \\
& \leq|| \lambda\left|\sum_{j=1}^{N}\left\|A_{j}-B_{j}\right\|_{1} \exp \right| \lambda \mid\left(\sum_{j=1}^{N}\left(\left\|A_{j}\right\|_{\infty}+\left\|B_{j}\right\|_{\infty}\right)\right) \\
& \leq \frac{\mathrm{e} \sum_{j=1}^{N}\left\|A_{j}-B_{j}\right\|_{1}}{2 \max \left(\sum_{j=1}^{N}\left\|A_{j}\right\|_{\infty}, \sum_{j=1}^{N}\left\|B_{j}\right\|_{\infty}\right)} .
\end{aligned}
$$

By substituting (5.8), (5.9) and (5.10) into (5.7) and using the result and the value of $\rho$ to estimate the integral (5.6), we obtain the statement.

5.3. A comparison principle. In the next step, we will prove a comparison principle for $C_{k}^{(n)}\left(A_{1}, \ldots, A_{N}\right)$ in case the $A_{j}$ are banded matrices.

We start with an easy lemma.

LEMMA 5.4. Let $N \in \mathbb{N}$ and $A_{1}, \ldots, A_{N}$ banded matrices with $\left(A_{j}\right)_{r s}=0$ if $|r-s|>a_{j}$. Then $A_{1} \cdots A_{N}$ is a banded matrix such that $\left(A_{1} \cdots A_{N}\right)_{r s}=0$ if $|r-s|>a_{1}+\cdots+a_{N}$ and $\left(A_{1} \cdots A_{N}\right)_{r s}$ only depends on entries $\left(A_{j}\right)_{k \ell}$ with $|k-r|,|\ell-s| \leq a_{1}+\cdots+a_{N}$ for $j=1, \ldots, N$.

PROOF. Write

$$
\left(A_{1} \cdots A_{N}\right)_{r s}=\sum_{v_{1}, \ldots, v_{N-1}}\left(A_{1}\right)_{r v_{1}}\left(A_{2}\right)_{v_{1} v_{2}} \cdots\left(A_{N}\right)_{v_{T-1} s} .
$$

By the assumption of the lemma, each term in the sum can only be nonzero if $\left|v_{j-1}-v_{j}\right| \leq a_{j}$ for $j=1, \ldots, N$ (where we have set $v_{0}=r$ and $v_{N}=s$ for notational convenience). But then by the triangular inequality, we see that the only possibility of obtain a nonzero value is in case $|r-s| \leq a_{1}+\cdots+a_{N}$, which proves the first part of the statement. Moreover, we only have contribution of entries $\left(A_{j}\right)_{v_{j-1} v_{j}}$ with

$$
\left|r-v_{j-1}\right| \leq\left|r-v_{1}\right|+\cdots+\left|v_{j-2}-v_{j-1}\right| \leq a_{1}+\cdots+a_{N}
$$

and

$$
\left|r-v_{j}\right| \leq\left|r-v_{j}\right|+\cdots+\left|v_{N}-v_{N-1}\right| \leq a_{1}+\cdots+a_{N},
$$


which proves the second part of the lemma.

The following is the core of the proof of the main results of this paper.

Proposition 5.5. Let $N \in \mathbb{N}$ and $A_{1}, \ldots, A_{N}$ be banded matrices such that $\left(A_{j}\right)_{r s}=0$ if $|r-s|>a_{j}$. Set $a=\sup _{j=1, \ldots, N} a_{j}$. Then

$$
C_{k}^{(n)}\left(A_{1}, \ldots, A_{N}\right)=C_{k}^{(n)}\left(R_{n, a(k+1)} A_{1} R_{n, a(k+1)}, \ldots, R_{n, 2 a(k+1)} A_{N} R_{n, 2 a(k+1)}\right),
$$

where

$$
R_{n, \ell}=P_{n+\ell}-P_{n-\ell}
$$

Proof. Note that since each $\lambda P_{n} A_{m} P_{n}$ is trace class [and for trace class operators $A, B$ we have $\operatorname{det}\left(\mathrm{e}^{A} \mathrm{e}^{B}\right)=\operatorname{det} \mathrm{e}^{A+B}=\mathrm{e}^{\operatorname{Tr}(A+B)}$ ], we can write

$$
\begin{aligned}
\lambda \operatorname{Tr} & \left(P_{n} A_{1} P_{n}+\cdots+P_{n} A_{N} P_{n}\right) \\
& =\log \operatorname{det}\left(\mathrm{e}^{\lambda P_{n} A_{1} P_{n}} \mathrm{e}^{\lambda P_{n} A_{2} P_{n}} \cdots \mathrm{e}^{\lambda P_{n} A_{N} P_{n}}\right) \\
& =\log \operatorname{det}\left(I+\left(\mathrm{e}^{\lambda P_{n} A_{1} P_{n}} \mathrm{e}^{\lambda P_{n} A_{2} P_{n}} \cdots \mathrm{e}^{\lambda P_{n} A_{N} P_{n}}-I\right)\right) .
\end{aligned}
$$

By expanding the right-hand side in the same way as in the proof of Lemma 5.2 and comparing terms at both sides, we find the identity:

$$
\sum_{j=1}^{k} \frac{(-1)^{j}}{j} \sum_{\substack{\ell_{1}+\cdots+\ell_{j}=k \\ \ell_{i} \geq 1}} \sum_{\substack{r_{s, v} \\ \text { en }}} \frac{\operatorname{Tr} \prod_{\ell_{1}, \ldots, \ell_{j}}^{j}}{r_{1,1} ! \cdots r_{1, N} ! r_{2,1} ! \cdots r_{2, N} ! \cdots r_{j, 1} ! \cdots r_{j, N} !}=0,
$$

for $k \geq 2$. But then we can write

$$
\begin{aligned}
C_{k}^{(n)}\left(A_{1}, \ldots, A_{N}\right) & \sum_{j=1}^{m} \frac{(-1)^{j+1}}{j} \sum_{\substack{\ell_{1}+\cdots+\ell_{j}=k \\
\ell_{i} \geq 1}} \sum_{\substack{\left.r_{s, v}\right) \in R_{\ell_{1}, \ldots, \ell_{j}}\\
}} \\
& \frac{\operatorname{Tr}\left(\prod_{s=1}^{j}\left(P_{n} A_{1}^{r_{s, 1}} \cdots A_{N}^{r_{s, N}} P_{n}\right)-\prod_{s=1}^{j} \prod_{u=1}^{N}\left(P_{n} A_{u} P_{n}\right)^{r_{s, u}}\right)}{r_{1,1} ! \cdots r_{1, N} ! r_{2,1} ! \cdots r_{2, N} ! \cdots r_{j, 1} ! \cdots r_{j, N} !},
\end{aligned}
$$

for $m \geq 2$. We prove the theorem by showing that the each summand only depends on some entries of $A_{j}$ that are all centered around the $n n$-entries.

Note that by a telescoping series, we have

$$
A_{p}^{r_{\ell, p}}-\left(P_{n} A_{p} P_{n}\right)^{r_{\ell, p}}=\sum_{q=0}^{r_{\ell, p}-1} A_{p}^{r_{\ell, p}-q-1}\left(A_{p}-P_{n} A_{p} P_{n}\right)\left(P_{n} A_{p} P_{n}\right)^{q}
$$


and

$$
\begin{aligned}
P_{n}\left(\prod_{u=1}^{N} A_{u}^{r_{\ell, u}}\right) P_{n}-\prod_{u=1}^{N}\left(P_{n} A_{u} P_{n}\right)^{r_{\ell, u}} \\
=\sum_{p=1}^{N} P_{n}\left(\prod_{u=1}^{p-1} A_{u}^{r_{\ell, u}}\right)\left(A_{p}^{r_{\ell, p}}-\left(P_{n} A_{p} P_{n}\right)^{r_{\ell, p}}\right)\left(\prod_{v=p+1}^{N}\left(P_{n} A_{v} P_{n}\right)^{r_{\ell, v}}\right) P_{n} \\
=\sum_{p=1}^{N} \sum_{q=0}^{r_{\ell, p}-1} P_{n}\left(\prod_{u=1}^{p-1} A_{u}^{r_{\ell, u}}\right) A_{p}^{r_{\ell, p}-q-1}\left(A_{p}-P_{n} A_{p} P_{n}\right)\left(P_{n} A_{p} P_{n}\right)^{q} \\
\quad \times\left(\prod_{v=p+1}^{N}\left(P_{n} A_{v} P_{n}\right)^{r_{\ell, v}}\right) P_{n} .
\end{aligned}
$$

We use the fact that $P_{n}^{2}=P_{n}$ to rewrite this to

$$
\begin{aligned}
P_{n}\left(\prod_{u=1}^{N} A_{u}^{r_{\ell, u}}\right) P_{n}-\prod_{u=1}^{N}\left(P_{n} A_{u} P_{n}\right)^{r_{\ell, u}} \\
\quad=\sum_{p=1}^{N} \sum_{q=0}^{r_{\ell, p}-1} P_{n}\left(\prod_{u=1}^{p-1} A_{u}^{r_{\ell, u}}\right) A_{p}^{r_{\ell, p}-q-1}\left(A_{p} P_{n}-P_{n} A_{p} P_{n}\right)\left(P_{n} A_{p} P_{n}\right)^{q} \\
\quad \times\left(\prod_{v=p+1}^{N}\left(P_{n} A_{v} P_{n}\right)^{r_{\ell, v}}\right) .
\end{aligned}
$$

Finally, by another telescoping series we find

$$
\begin{aligned}
\prod_{s=1}^{j}\left(P_{n} A_{1}^{r_{s, 1}} \cdots A_{n}^{r_{s, N}} P_{n}\right)-\prod_{s=1}^{j}\left(P_{n}\left(A_{1} P_{n}\right)^{r_{s, 1}} \cdots\left(A_{N} P_{n}\right)^{r_{s, N}}\right) \\
=\sum_{\ell=1}^{j} \sum_{p=1}^{N} \sum_{q=0}^{r_{\ell, p}-1}\left(\prod_{s=1}^{\ell-1}\left(P_{n} A_{1}^{r_{s, 1}} \cdots A_{N}^{r_{s, N}} P_{n}\right) P_{n}\left(\prod_{u=1}^{p-1} A_{u}^{r_{\ell, u}}\right)\right) \\
\quad \times\left(A_{p}^{r_{\ell, p}-q-1}\left(A_{p} P_{n}-P_{n} A_{p} P_{n}\right)\left(P_{n} A_{p} P_{n}\right)^{q}\right) \\
\quad \times\left(\prod_{v=p+1}^{N}\left(P_{n} A_{v} P_{n}\right)^{r_{\ell, v}}\right)\left(\prod_{s=\ell+1}^{j}\left(P_{n}\left(A_{1} P_{n}\right)^{r_{s, 1}} \cdots\left(P_{n} A_{N} P_{n}\right)^{r_{s, N}}\right)\right) \\
=\sum_{\ell=1}^{j} \sum_{p=1}^{N} \sum_{q=0}^{r_{\ell, p}-1} \operatorname{Tr} Q_{1}\left(A_{p} P_{n}-P_{n} A_{p} P_{n}\right) Q_{2}
\end{aligned}
$$


with

$$
\begin{aligned}
Q_{1}= & \left(\prod_{s=1}^{\ell-1}\left(P_{n} A_{1}^{r_{s, 1}} \cdots A_{N}^{r_{s, N}} P_{n}\right)\left(\prod_{u=1}^{p-1} A_{u}^{r_{\ell, u}}\right)\right) A_{p}^{r_{k, p}-q-1}, \\
Q_{2}= & \left(P_{n} A_{p} P_{n}\right)^{q}\left(\prod_{v=p+1}^{N}\left(P_{n} A_{v} P_{n}\right)^{r_{k, v}}\right) \\
& \times\left(\prod_{s=\ell+1}^{j}\left(P_{n}\left(A_{1} P_{n}\right)^{r_{s, 1}} \cdots\left(P_{n} A_{N} P_{n}\right)^{r_{s, N}}\right)\right) .
\end{aligned}
$$

Let us compute

$$
\begin{aligned}
\operatorname{Tr} Q_{1} & \left(A_{p} P_{n}-P_{n} A_{p} P_{n}\right) Q_{2} \\
& =\sum_{r_{0}=1}^{n} \sum_{r_{1}, r_{2}}\left(Q_{1}\right)_{r_{0} r_{1}}\left(A_{p} P_{n}-P_{n} A_{p} P_{n}\right)_{r_{1} r_{2}}\left(Q_{2}\right)_{r_{2} r_{0}} .
\end{aligned}
$$

The fact of the matter is that because of the band structure the matrix

$$
A_{p} P_{n}-P_{n} A_{p} P_{n}
$$

is of finite rank and the nonzero entries are concentrated around the $n n$-entry. Hence we can restrict the sum to terms with $\left|r_{1,2}-n\right| \leq a$. Now, since $Q_{1}$ and $Q_{2}$ are a product of band matrices, they are themselves also band matrices. The number of terms in the product is at most $k$ (ignoring the $P_{n}$ ) and the bandwidth of each term is at most $a$. Hence $\left(Q_{1}\right)_{r_{0} r_{1}}=0$ if $\left|r_{0}-r_{1}\right|>a k$ and $\left(Q_{2}\right)_{r_{2} r_{0}}=0$ if $\left|r_{2}-r_{0}\right|>a k$. By combining the latter observations, we see that the trace in (5.11) only depends on $\left(Q_{1}\right)_{r_{0} r_{1}}$ and $\left(Q_{1}\right)_{r_{2} r_{0}}$ with $\left|r_{0}-n\right| \leq a(k+1)$ and $\left|r_{1,2}-n\right| \leq a$. By Lemma 5.4, we then also see that these entries only depend on entries $\left(A_{m}\right)_{r s}$ with $|r-n| \leq a(k+1)$ and $|s-n| \leq a(k+1)$ for $m=1, \ldots, N$. Concluding, we have that $C_{m}^{(n)}\left(A_{1}, \ldots, A_{N}\right)$ only depends on $\left(A_{m}\right)_{r s}$ with $|r-n| \leq 2 a(k+1)$ and $|s-n| \leq 2 a(k+1)$, for $m=1, \ldots, N$. This proves the statement.

Corollary 5.6. Let $N \in \mathbb{N}$ and $A_{1}, \ldots, A_{N}, B_{1}, \ldots, B_{N}$ be banded matrices such that $\left(A_{j}\right)_{r s}=0$ if $|r-s|>a_{j}$ and $\left(B_{j}\right)_{r s}=0$ if $|r-s|>b_{j}$. Set 
$c=\max \left\{a_{j}, b_{j} \mid j=1, \ldots, N\right\}$. Then

$$
\begin{aligned}
&\left|C_{k}^{(n)}\left(A_{1}, \ldots, A_{N}\right)-C_{k}^{(n)}\left(B_{1}, \ldots, B_{N}\right)\right| \\
& \leq\left(\operatorname { m a x } \left(\sum_{j=1}^{N}\left\|R_{n, 2 c(k+1)} A_{j} R_{n, 2 c(k+1)}\right\|_{\infty},\right.\right. \\
&\left.\left.\sum_{j=1}^{N}\left\|R_{n, 2 c(k+1)} B_{j} R_{n, 2 c(k+1)}\right\|_{\infty}\right)\right)^{k-1} \\
& \times \frac{2^{k+2} c(k+1) \mathrm{e}}{(2-\sqrt{\mathrm{e}})^{2}} \sum_{j=1}^{N}\left\|R_{n, 2 c(k+1)}\left(A_{j}-B_{j}\right) R_{n, 2 c(k+1)}\right\|_{\infty},
\end{aligned}
$$

where $R_{n, 2 c(k+1)}$ is as in Proposition 5.5.

ProOF. By combining Lemma 5.3 and Proposition 5.5, we obtain

$$
\begin{aligned}
\mid C_{k}^{(n)}( & \left.A_{1}, \ldots, A_{N}\right)-C_{k}^{(n)}\left(B_{1}, \ldots, B_{N}\right) \mid \\
\leq & \left(2 \operatorname { m a x } \left(\sum_{j=1}^{N}\left\|R_{n, 2 c(k+1)} A_{j} R_{n, 2 c(k+1)}\right\|_{\infty},\right.\right. \\
& \left.\left.\sum_{j=1}^{N}\left\|R_{n, 2 c(k+1)} B_{j} R_{n, 2 c(k+1)}\right\|_{\infty}\right)\right)^{k-1} \frac{2 \mathrm{e}}{(2-\sqrt{\mathrm{e}})^{2}} \\
& \times \sum_{j=1}^{N}\left\|R_{n, 2 c(k+1)} A_{j} R_{n, 2 c(k+1)}-R_{n, 2 c(k+1)} B_{j} R_{n, 2 c(k+1)}\right\|_{1} .
\end{aligned}
$$

Now the statement follows by noting that the ranks of

$$
R_{n, 2 c(k+1)} A_{j} R_{n, 2 c(k+1)} \quad \text { and } \quad R_{n, 2 c(k+1)} B_{j} R_{n, 2 c(k+1)}
$$

are $4 c(k+1)+1$ and for any finite rank operator $R$ with rank $r(R)$ we have $\|R\|_{1} \leq r(R)\|R\|_{\infty}$.

Note that the latter corollary is a pure universality result. Whatever the limits are, they must be the same. It particularly implies that we only need to compute a special case to conclude a general result. This is what we will do in the next paragraph.

5.4. Special case of banded Toeplitz operators. We now compute the limiting values of $C_{m}^{(n)}\left(A_{1}, \ldots, A_{N}\right)$ in case the $A_{j}$ are banded Toeplitz operators. We will first recall various basic notions and properties we will need. For further details and background on Toeplitz operators, we refer to the book [11]. 
For a Laurent polynomial $a(z)=\sum_{j=-q}^{p} a_{j} z^{j}$, the Toeplitz operator $T(a)$ is defined by the semi-infinite matrix:

$$
(T(a))_{j k}=a_{j-k}, \quad j, k=1, \ldots,
$$

viewed as an operator on $\ell_{2}(\mathbb{N})$. Of importance to us will also be the Hankel operator defined by the semi-infinite matrix:

$$
(H(a))_{j k}=a_{j+k-1}, \quad j, k=1, \ldots
$$

Note that $H(a)$ is of finite rank. The Toeplitz and Hankel operators are related by

$$
T(a b)=T(a) T(b)+H(a) H(\tilde{b}),
$$

with $\tilde{b}(z)=b(1 / z)$. An important consequence of this formula that we will frequently use is

$$
[T(a), T(b)]=H(b) H(\tilde{a})-H(a) H(\tilde{b}) .
$$

Finally, we mention that

$$
\|T(a)\|_{\infty} \leq\|a\|_{\infty} \quad \text { and } \quad\|H(a)\|_{\infty} \leq\|a\|_{\infty},
$$

where $\|a\|_{\infty}$ denotes $\mathbb{L}_{\infty}$-norm of $a$, which by continuity is simply the maximum of $a$.

5.4.1. The case $N$ fixed. The main purpose of this paragraph is to prove the following proposition.

Proposition 5.7. Let $N \in \mathbb{N}$ and $a(m, z)=\sum_{\ell} a_{\ell}(m) z^{\ell}$ for $m=1, \ldots, N$, be Laurent polynomials in $z$. For $m=1, \ldots, N$, we denote the Toeplitz operator with symbol $a(m, z)$ by $T(a(m))$. Then

$$
\begin{aligned}
\lim _{n \rightarrow \infty} \operatorname{det}\left(I+P_{n}\left(\mathrm{e}^{T(a(1))} \mathrm{e}^{T(a(2))} \cdots \mathrm{e}^{T(a(N))}-I\right) P_{n}\right) \mathrm{e}^{-n \sum_{m=1}^{N} a_{0}(m)} \\
=\exp \left(\sum_{m_{1}=1}^{N} \sum_{m_{2}=m_{1}+1}^{N} \sum_{\ell=1}^{\infty} \ell a_{\ell}\left(m_{1}\right) a_{-\ell}\left(m_{2}\right)\right. \\
\left.+\frac{1}{2} \sum_{m=1}^{N} \sum_{\ell=1}^{\infty} \ell a_{\ell}(m) a_{-\ell}(m)\right) .
\end{aligned}
$$

Before we come to the proof, we first mention that the following immediate corollary.

COROLlARY 5.8. Under the same assumptions and notation as in Proposition 5.7, we have

$$
\lim _{n \rightarrow \infty} C_{k}^{(n)}(T(a(1)), \ldots, T(a(N)))=0,
$$


for $k \geq 3$, and

$$
\begin{aligned}
\lim _{n \rightarrow \infty} C_{k}^{(n)}(T(a(1)), \ldots, T(a(N))) \\
\quad=\sum_{m_{1}=1}^{N} \sum_{m_{2}=m_{1}+1}^{N} \sum_{\ell=1}^{\infty} \ell a_{\ell}\left(m_{1}\right) a_{-\ell}\left(m_{2}\right)+\frac{1}{2} \sum_{m=1}^{N} \sum_{\ell=1}^{\infty} \ell a_{\ell}(m) a_{-\ell}(m)
\end{aligned}
$$

for $k=2$.

The proof of Proposition 5.7 that we will present here relies on the following beautiful identity due to Ehrhardt.

LEMmA 5.9 ([23], Corollary 2.3). Let $A_{1}, \ldots, A_{N}$ be bounded operators such that

1. $A_{1}+\cdots+A_{N}=0$,

2. $\left[A_{i}, A_{j}\right]$ is trace class for $1 \leq i<j \leq N$.

Then $\mathrm{e}^{A_{1}} \mathrm{e}^{A_{2}} \cdots \mathrm{e}^{A_{N}}-I$ is of trace class and

$$
\operatorname{det} \mathrm{e}^{A_{1}} \mathrm{e}^{A_{2}} \ldots \mathrm{e}^{A_{N}}=\exp \frac{1}{2} \sum_{1 \leq i<j \leq n} \operatorname{Tr}\left[A_{i}, A_{j}\right],
$$

where the left-hand side is a Fredholm determinant.

REMARK 5.1. In the special case $n=3$, the identity reads

$$
\operatorname{det} \mathrm{e}^{-A} \mathrm{e}^{A+B} \mathrm{e}^{-B}=\exp \left(-\frac{1}{2} \operatorname{Tr}[A, B]\right),
$$

which is an identity that lies behind the Strong Szegő Limit Theorem. It has also been used in [13] in the context of Central Limit Theorem for linear statistics for bi-orthogonal ensembles.

We now come to the following.

Proof of Proposition 5.7. We start by defining $s(z)=\sum_{j=1}^{N} a(j, z)$ and split $s(z)=s_{+}(z)+s_{-}(z)$ where $s_{+}$is the polynomial part of $s(z)$, that is, $s_{+}(z)=$ $\sum_{k \geq 0} s_{k} z^{k}$. Then we note that $T\left(s_{+}\right)$is lower triangular and $T\left(s_{-}\right)$is strictly upper triangular. Hence

$$
P_{n} T\left(s_{+}\right) P_{n}=P_{n} T\left(s_{+}\right) \quad \text { and } \quad P_{n} T\left(s_{-}\right) P_{n}=T\left(s_{-}\right) P_{n} .
$$

By expanding the exponential and iterating the latter identities we therefore have (where we recall that $Q_{n}=I-P-n$ )

$$
\mathrm{e}^{P_{n} T\left(s_{+}\right) P_{n}}=P_{n} \mathrm{e}^{T\left(s_{+}\right)} P_{n}+Q_{n}=P_{n} \mathrm{e}^{T\left(s_{+}\right)}+Q_{n},
$$


and

$$
\mathrm{e}^{P_{n} T\left(s_{-}\right) P_{n}}=P_{n} \mathrm{e}^{T\left(s_{-}\right)} P_{n}+Q_{n}=\mathrm{e}^{T\left(s_{-}\right)} P_{n}+Q_{n} .
$$

Moreover,

$$
\begin{aligned}
\left(Q_{n}\right. & \left.+P_{n} \mathrm{e}^{T\left(s_{+}\right)} P_{n}\right)\left(Q_{n}+P_{n} B P_{n}\right)\left(Q_{n}+P_{n} \mathrm{e}^{T\left(s_{-}\right)} P_{n}\right) \\
& =Q_{n}+P_{n} \mathrm{e}^{T\left(s_{+}\right)} P_{n} B P_{n} \mathrm{e}^{T\left(s_{-}\right)} P_{n} \\
& =Q_{n}+P_{n} \mathrm{e}^{T\left(s_{+}\right)} B \mathrm{e}^{T\left(s_{-}\right)} P_{n} \\
& =I+P_{n}\left(\mathrm{e}^{T\left(s_{+}\right)} B \mathrm{e}^{T\left(s_{-}\right)}-I\right) P_{n},
\end{aligned}
$$

for any operator $B$. We then write

$$
\mathrm{e}^{-n \sum_{j=1}^{N} a_{0}^{(j)}}=\mathrm{e}^{-\operatorname{Tr} P_{n} T\left(s_{+}\right) P_{n}}=\operatorname{det} \mathrm{e}^{-P_{n} T\left(s_{+}\right) P_{n}}=\operatorname{det}\left(Q_{n}+P_{n} \mathrm{e}^{-T\left(s_{+}\right)} P_{n}\right),
$$

and

$$
1=\mathrm{e}^{-\operatorname{Tr} P_{n} T\left(s_{-}\right) P_{n}}=\operatorname{det} \mathrm{e}^{-P_{n} T\left(s_{-}\right) P_{n}}=\operatorname{det}\left(Q_{n}+P_{n} \mathrm{e}^{-T\left(s_{-}\right)} P_{n}\right) .
$$

By combining this with (5.15) and taking $B=\mathrm{e}^{T(a(1))} \mathrm{e}^{T(a(2))} \cdots \mathrm{e}^{T(a(N))}$, we see that we can rewrite the left-hand side of (5.14) as

$$
\operatorname{det}\left(I+P_{n}\left(\mathrm{e}^{-T\left(s_{+}\right)} \mathrm{e}^{T(a(1))} \mathrm{e}^{T(a(2))} \cdots \mathrm{e}^{T(a(N))} \mathrm{e}^{-T\left(s_{-}\right)}-I\right) P_{n}\right) .
$$

The idea is now to invoke Lemma 5.9. To this end, we first note that

$$
T(s)+\sum_{j=1}^{N} T\left(a^{(j)}\right)=0,
$$

and that for $1 \leq j, k \leq N$ we have that

$$
\left[T\left(a_{+}(j)\right), T\left(a_{-}(k)\right)\right]=H\left(a_{+}(j)\right) H\left(\tilde{a}_{-}(k)\right),
$$

is of finite rank, and hence of trace class, from which it follows that also the second condition of the proposition is satisfied and that

$$
\mathrm{e}^{-T\left(s_{+}\right)} \mathrm{e}^{T(a(1))} \mathrm{e}^{T(a(2))} \cdots \mathrm{e}^{T(a(N))} \mathrm{e}^{-T\left(s_{-}\right)}-I
$$

is of trace class. Hence

$$
\begin{gathered}
P_{n}\left(\mathrm{e}^{-T\left(s_{+}\right)} \mathrm{e}^{T(a(1))} \mathrm{e}^{T(a(2))} \cdots \mathrm{e}^{T(a(N))} \mathrm{e}^{-T\left(s_{-}\right)}-I\right) P_{n} \\
\quad \rightarrow \mathrm{e}^{-T\left(s_{+}\right)} \mathrm{e}^{T(a(1))} \mathrm{e}^{T(a(2))} \cdots \mathrm{e}^{T(a(N))} \mathrm{e}^{-T\left(s_{-}\right)}-I,
\end{gathered}
$$

in trace norm. By continuity of the Fredholm determinant, we can therefore take the limit $n \rightarrow \infty$ and obtain

$$
\begin{gathered}
\lim _{n \rightarrow \infty} \operatorname{det}\left(I+P_{n}\left(\mathrm{e}^{-T\left(s_{+}\right)} \mathrm{e}^{T(a(1))} \mathrm{e}^{T(a(2))} \cdots \mathrm{e}^{T(a(N))} \mathrm{e}^{T\left(s_{-}\right)}-I\right) P_{n}\right) \\
=\operatorname{det} \mathrm{e}^{T\left(s_{+}\right)} \mathrm{e}^{T(a(1))} \mathrm{e}^{T(a(2))} \cdots \mathrm{e}^{T(a(N))} \mathrm{e}^{-T\left(s_{-}\right)} .
\end{gathered}
$$


Moreover, by the same proposition,

$$
\operatorname{det} \mathrm{e}^{-T\left(s_{+}\right)} \mathrm{e}^{T(a(1))} \mathrm{e}^{T(a(2))} \cdots \mathrm{e}^{T(a(N))} \mathrm{e}^{-T\left(s_{-}\right)}=\exp \frac{1}{2} \operatorname{Tr} S_{1},
$$

with

$$
\begin{aligned}
S_{1}= & -\sum_{j=1}^{N}\left[T\left(s_{+}\right), T\left(a^{(j)}\right)\right]+\left[T\left(s_{+}\right), T\left(s_{-}\right)\right] \\
& +\sum_{j=1}^{N} \sum_{k>j}[T(a(j)), T(a(k))]-\sum_{j=1}^{N}\left[T(a(j)), T\left(s_{-}\right)\right] .
\end{aligned}
$$

By splitting $a(m)=a_{+}(m)+a_{-}(m)$ and using the definition of $s_{ \pm}$, we can rewrite this to

$$
\begin{aligned}
S_{1} & =\sum_{j=1}^{N} \sum_{k>j}[T(a(j)), T(a(k))]-\sum_{j=1}^{N} \sum_{k=1}^{N}\left[T(a(j)), T\left(a_{-}(k)\right)\right] \\
& =\sum_{j=1}^{N} \sum_{k>j}\left[T(a(j)), T\left(a_{+}(k)\right)\right]-\sum_{j=1}^{N} \sum_{k \leq j}\left[T(a(j)), T\left(a_{-}(k)\right)\right] \\
& =\sum_{j=1}^{N} \sum_{k>j} H(a(k)) H(\tilde{a}(j))+\sum_{j=1}^{N} \sum_{k \leq j} H(a(j)) H(\tilde{a}(k)),
\end{aligned}
$$

where in the last step we used (5.13). By taking the trace, we find

$$
\begin{aligned}
\operatorname{Tr} S_{1} & =\sum_{j=1}^{N} \sum_{k=j+1}^{N} \sum_{\ell=1}^{\infty} \ell a_{\ell}(k) a_{-\ell}(j)+\sum_{j=1}^{N} \sum_{k=1}^{j} \sum_{\ell=1}^{\infty} \ell a_{\ell}(j) a_{-\ell}(k) \\
& =2 \sum_{j=1}^{N} \sum_{k=j+1}^{N} \sum_{\ell=1}^{\infty} \ell a_{\ell}(k) a_{-\ell}(j)+\sum_{j=1}^{N} \sum_{\ell=1}^{\infty} \ell a_{\ell}(j) a_{-\ell}(j) .
\end{aligned}
$$

Hence the statement follows after inserting the latter expression for $\operatorname{Tr} S_{1}$ into (5.19) and combining the result with (5.18).

5.4.2. The case $N_{n} \rightarrow \infty$. We now come to the case that $N=N_{n}$ is depending on $n$ in such a way that $N_{n} \rightarrow \infty$ as $n \rightarrow \infty$. In this paragraph, we prove the following proposition.

Proposition 5.10. Let $a(t, z)=\sum_{j=-q}^{p} a_{j}(t) z^{j}$ be a $t$ dependent Laurent polynomial for which the $a_{j}(t)$ are piecewise continuous on an interval $[\alpha, \beta]$. Let $\left\{N_{n}\right\}_{n \in \mathbb{N}}$ be a sequence such that $N_{n} \rightarrow \infty$ as $n \rightarrow \infty$. Moreover, for each $n$ let

$$
\alpha=t_{1}^{(n)}<t_{2}^{(n)}<\cdots<t_{N_{n}}^{(n)}<t_{N_{n}+1}^{(n)}=\beta
$$


be a partitioning of $[\alpha, \beta]$ for which the mesh $\sup _{j=0, \ldots, N_{n}}\left(t_{j+1}-t_{j}\right) \rightarrow 0$ as $n \rightarrow \infty$. Then

$$
\begin{gathered}
\operatorname{det}\left(I+P_{n}\left(\prod_{m=1}^{N_{n}} \mathrm{e}^{\left(t_{m+1}-t_{m}\right) T\left(a\left(t_{m}^{(n)}\right)\right)}-I\right) P_{n}\right) \mathrm{e}^{-\sum_{m=1}^{N_{n}}\left(t_{m+1}-t_{m}\right) a_{0}\left(t_{m}^{(n)}\right)} \\
=\exp \left(\sum_{\ell=1}^{\infty} \iint_{\alpha<t_{1}<t_{2}<\beta} \ell a_{\ell}\left(t_{1}\right) a_{-\ell}\left(t_{2}\right) \mathrm{d} t_{1} \mathrm{~d} t_{2}\right) .
\end{gathered}
$$

COROLlARY 5.11. Under the same assumptions and notation as in Proposition 5.10, we have

$$
\lim _{n \rightarrow \infty} C_{k}^{(n)}\left(T(a(1)), \ldots, T\left(a\left(N_{n}\right)\right)\right)=0,
$$

for $k \geq 3$, and

$$
\lim _{n \rightarrow \infty} C_{k}^{(n)}(T(a(1)), \ldots, T(a(N)))=2 \sum_{\ell=1}^{\infty} \iint_{\alpha<t_{1}<t_{2}<\beta} \ell a_{\ell}\left(t_{1}\right) a_{-\ell}\left(t_{2}\right) \mathrm{d} t_{1} \mathrm{~d} t_{2},
$$

for $k=2$.

The proof of Proposition 5.10 goes along the same lines as the proof of Proposition 5.7. The main difficulty is that (5.17) (with $N$ and the symbols depending on $n$ ) is no longer immediate and requires a proof. Hence we cannot deduce (5.18). We overcome this issue by proving the following.

LEMMA 5.12. Let $\left\{N_{n}\right\}_{n}$ be a sequence of integers. For each $n \in \mathbb{N}$ let $A_{j}^{(n)}$ for $j=1, \ldots, N_{n}$ be a family of Toeplitz operators satisfying the following conditions

- $A_{1}^{(n)}+\cdots+A_{N_{n}}^{(n)}=0$,

- the $A_{j}^{(n)}$ 's are banded with width $c$ which is independent of $j$ and $n$,

- $\sum_{j=1}^{N_{n}}\left\|A_{j}^{(n)}\right\|_{\infty}<r$, for some constant $r$ independent of $n$.

Then

$$
\lim _{n \rightarrow \infty}\left\|P_{n}\left(\mathrm{e}^{A_{1}^{(n)}} \cdots \mathrm{e}^{A_{N_{n}}^{(n)}}-I\right) P_{n}-\left(\mathrm{e}^{A_{1}^{(n)}} \cdots \mathrm{e}^{A_{N_{n}}^{(n)}}-I\right)\right\|_{1}=0 .
$$

ProOF. Note that

$$
\begin{aligned}
& P_{n}\left(\mathrm{e}^{A_{1}^{(n)}} \cdots \mathrm{e}^{A_{N_{n}}^{(n)}}-I\right) P_{n}-\left(\mathrm{e}^{A_{1}^{(n)}} \cdots \mathrm{e}^{A_{N_{n}}^{(n)}}-I\right) \\
& \quad=-Q_{n}\left(\mathrm{e}^{A_{1}^{(n)}} \cdots \mathrm{e}^{A_{N_{n}}^{(n)}}-I\right) P_{n}-\left(\mathrm{e}^{A_{1}^{(n)}} \cdots \mathrm{e}^{A_{N_{n}}^{(n)}}-I\right) Q_{n} .
\end{aligned}
$$


Hence it suffices to prove that the two terms at the right-hand side separately converge to zero in trace norm. Here, we will only show that for the second term, that is, we show that

$$
\lim _{n \rightarrow \infty}\left\|\left(\mathrm{e}^{A_{1}^{(n)}} \cdots \mathrm{e}^{A_{N_{n}}^{(n)}}-I\right) Q_{n}\right\|_{1} \rightarrow 0 .
$$

The arguments for first term are analogous and left to the reader.

We first claim that for $A_{j}$ and $r, c$ satisfying the stated conditions, we have

$$
\left\|\left(\mathrm{e}^{A_{1}} \cdots \mathrm{e}^{A_{N}}-I\right) Q_{n}\right\|_{1} \leq \mathrm{e}^{2 r} \sum_{2 \leq s<t \leq N}\left\|\left[A_{s}, A_{t}\right]\right\|_{1} \sum_{m_{2}+m_{3} \geq n /(2 c)} \frac{r^{m_{2}+m_{3}}}{m_{2} ! m_{3} !},
$$

for any $n \geq 2 c$ and $N \in \mathbb{N}$.

The proof of this statement goes by induction to $N$.

We start with $N=3$. In that case, we recall a result from [12], Lemma 4.2, that if $A_{1}+A_{2}+A_{3}=0$ then

$$
\mathrm{e}^{A_{1}} \mathrm{e}^{A_{2}} \mathrm{e}^{A_{3}}-I=\sum_{m_{1}=0}^{\infty} \sum_{m_{2}=1}^{\infty} \sum_{m_{3}=0}^{\infty} \sum_{j=0}^{m_{2}-j-1} \frac{A_{1}^{m_{1}} A_{2}^{j}\left[A_{2}, A_{3}\right] A_{2}^{m_{2}-j-1} A_{3}^{m_{3}}}{m_{1} ! m_{2} ! m_{3} !\left(m_{1}+m_{2}+m_{3}+1\right)}
$$

(this follows easily after differentiating $\mathrm{e}^{t A_{1}} \mathrm{e}^{t A_{2}} \mathrm{e}^{t A_{3}}$ with respect to $t$, expanding the exponentials and then integrate again for $t$ from 0 to 1 ). Since $A_{j}$ are banded with bandwidth $c$, we also have $A_{2}^{m_{2}-j-1} A_{3}^{m_{3}}$ is banded with bandwidth $c\left(m_{2}+\right.$ $\left.m_{3}-j-1\right)$ but that means that

$$
A_{2}^{m_{2}-j-1} A_{3}^{m_{3}} Q_{n}=Q_{n-c\left(m_{2}+m_{3}-j-1\right)} A_{2}^{m_{2}-j-1} A_{3}^{m_{3}} Q_{n}
$$

and thus

$$
\left[A_{2}, A_{3}\right] A_{2}^{m_{2}-j-1} A_{3}^{m_{3}} Q_{n}=\left[A_{2}, A_{3}\right] Q_{n-c\left(m_{2}+m_{3}-j-1\right)} A_{2}^{m_{2}-j-1} A_{3}^{m_{3}} Q_{n} .
$$

Since by assumption $A_{2}$ and $B_{3}$ are banded Toeplitz matrices of bandwith $c$, we see by (5.13) that $\left[A_{1}, A_{2}\right]$ is sum of product of two Hankel matrices with Laurent polynomials as their symbols. All the nontrivial entries for these Hankel operators are in the upper left $c \times c$ block, and hence

$$
\left[A_{2}, A_{3}\right] Q_{m}=0
$$

for $m \geq c$. Hence, for $n \geq 2 c$, we can restrict the sum to terms with $m_{2}+m_{3} \geq$ $n / 2 c$ and write

$$
\begin{aligned}
& \left\|\left(\mathrm{e}^{-A} \mathrm{e}^{A+B} \mathrm{e}^{-B}-I\right) Q_{n}\right\|_{1} \\
& =\left\|\sum_{\substack{m_{1}=0 \\
m_{2} \geq 1, m_{3} \geq 0, m_{2}+m_{2} \geq n / 2 c}}^{\infty} \sum_{j=0}^{m_{2}-j-1} \frac{\left(A_{1}\right)^{m_{1}}\left(A_{2}\right)^{j}\left[A_{2}, A_{3}\right]\left(A_{2}\right)^{m_{2}-j-1}\left(A_{3}\right)^{m_{3}} Q_{n}}{m_{1} ! m_{2} ! m_{3} !\left(m_{1}+m_{2}+m_{3}+1\right)}\right\|_{1}
\end{aligned}
$$




$$
\begin{aligned}
& \leq \sum_{m_{1}=0}^{\infty} \sum_{\substack{m_{2} \geq 1, m_{3} \geq 0, m_{2}+m_{2} \geq n / 2 c}} \sum_{j=0}^{m_{2}-j-1} \frac{\left\|A_{1}\right\|_{\infty}^{m_{1}}\left\|A_{2}\right\|_{\infty}^{j}\left\|\left[A_{1}, A_{2}\right]\right\|_{1}\left\|A_{2}\right\|_{\infty}^{m_{2}-j-1}\left\|A_{3}\right\|_{\infty}^{m_{3}}}{m_{1} ! m_{2} ! m_{3} !\left(m_{1}+m_{2}+m_{3}+1\right)} \\
& \leq \mathrm{e}^{r}\left\|\left[A_{2}, A_{3}\right]\right\|_{1} \sum_{m_{2}+m_{3} \geq n /(2 c)} \frac{r^{m_{2}+m_{3}}}{m_{2} ! m_{3} !},
\end{aligned}
$$

and this proves the statement for $N=3$ (with a slightly better bound).

Now suppose the statement is true for for $N-1 \geq 2$. We then first write

$$
\begin{aligned}
\mathrm{e}^{A_{1}} \mathrm{e}^{A_{2}} & \cdots \mathrm{e}^{A_{N}}-I \\
= & \mathrm{e}^{A_{1}} \mathrm{e}^{A_{1}} \mathrm{e}^{A_{2}} \cdots \mathrm{e}^{A_{N-2}} \mathrm{e}^{A_{N-1}+A_{N}}\left(\mathrm{e}^{-A_{N-1}-A_{N}} \mathrm{e}^{A_{N-1}} \mathrm{e}^{A_{N}}-I\right) \\
& +\mathrm{e}^{A_{1}} \mathrm{e}^{A_{2}} \cdots \mathrm{e}^{A_{N-2}} \mathrm{e}^{A_{N-1}+A_{N}}-I .
\end{aligned}
$$

The first term at the right-hand side can be estimate by as in the case $N=3$ giving

$$
\begin{aligned}
& \left\|\mathrm{e}^{A_{1}} \mathrm{e}^{A_{1}} \mathrm{e}^{A_{2}} \cdots \mathrm{e}^{A_{N-2}} \mathrm{e}^{A_{N-1}+A_{N}}\left(\mathrm{e}^{-A_{N-1}-A_{N}} \mathrm{e}^{A_{N-1}} \mathrm{e}^{A_{N}}-I\right)\right\|_{1} \\
& \leq \mathrm{e}^{\sum_{j=1}^{N}\left\|A_{j}\right\|_{\infty}}\left\|\mathrm{e}^{-A_{N-1}-A_{N}} \mathrm{e}^{A_{N-1}} \mathrm{e}^{A_{N}}-I\right\|_{1} \\
& \leq \mathrm{e}^{2 r}\left\|\left[A_{N-1}, A_{N}\right]\right\|_{1} \sum_{m_{2}+m_{3} \geq n /(2 c)} \frac{r^{m_{2}+m_{3}}}{m_{2} ! m_{3} !} .
\end{aligned}
$$

Moreover, since

$$
\sum_{j=1}^{N-2}\left\|A_{j}\right\|_{\infty}+\left\|A_{N-1}+A_{N}\right\|_{\infty}<\sum_{j=1}^{N}\left\|A_{j}\right\|_{\infty} \leq r
$$

and $A_{N-1}+A_{N}$ by linearity is also a Toeplitz operator with bandwidth $c$, we have by the induction hypothesis that we find that

$$
\begin{aligned}
& \left\|\mathrm{e}^{A_{1}} \mathrm{e}^{A_{2}} \cdots \mathrm{e}^{A_{N-2}} \mathrm{e}^{A_{N-1}+A_{N}}-I\right\| \\
& \leq \mathrm{e}^{2 r}\left(\sum_{1 \leq s<t \leq N_{n}-2}\left\|\left[A_{s}, A_{t}\right]\right\|_{1}+\sum_{s=1}^{N-2}\left\|\left[A_{s}, A_{N-1}+A_{N}\right]\right\|_{1}\right) \\
& \quad \times \sum_{m_{2}+m_{3} \geq n /(2 c)} \frac{r^{m_{2}+m_{3}}}{m_{2} ! m_{3} !} \\
& \leq \mathrm{e}^{2 r}\left(\sum_{1 \leq s<t \leq N_{n}-1}\left\|\left[A_{s}, A_{t}\right]\right\|_{1}+\sum_{s=1}^{N-2}\left\|\left[A_{s}, A_{N}\right]\right\|_{1}\right) \\
& \quad \times \sum_{m_{2}+m_{3} \geq n /(2 c)} \frac{r^{m_{2}+m_{3}}}{m_{2} ! m_{3} !} .
\end{aligned}
$$


Hence, by combining (5.23) and (5.24) we obtain the claim (5.22) for any $N \in \mathbb{N}$.

To complete the proof, we recall again that by (5.13) we have that $\left[A_{s}, A_{t}\right]$ is sum of product of two Hankel matrices with Laurent polynomials as their symbols. All the nontrivial entries for these Hankel operators are in the upper left $c \times c$ block, and hence they are of rank $c$. Since we also have $\|H(a)\|_{\infty} \leq\|T(a)\|_{\infty}$, we therefore find

$$
\left\|\left[A_{s}, A_{t}\right]\right\|_{1} \leq 2 c^{2}\left\|A_{s}\right\|_{\infty}\left\|A_{t}\right\|_{\infty}
$$

But then we have

$$
\sum_{2 \leq s<t \leq N}\left\|\left[A_{s}, A_{t}\right]\right\|_{1} \leq 2 c^{2} \sum_{2 \leq s<t \leq N}\left\|A_{s}\right\|_{\infty}\left\|A_{t}\right\|_{\infty}<r^{2} c^{2} .
$$

After inserting this into (5.22), we obtain

$$
\left\|\left(\mathrm{e}^{A_{1}} \cdots \mathrm{e}^{A_{N}}-I\right) Q_{n}\right\|_{1} \leq 2 r^{2} c^{2} \mathrm{e}^{2 r} \sum_{m_{2}+m_{3} \geq n /(2 c)} \frac{r^{m_{2}+m_{3}}}{m_{2} ! m_{3} !},
$$

for any $n \geq 2 c, N \in \mathbb{N}$ and operators $\left\{A_{j}\right\}_{j=1}^{N}$ satisfying the conditions of the proposition (with respect to $c$ and $r$ ). By setting $N=N_{n}, A_{j}=A_{j}^{(n)}$ and taking $n \rightarrow \infty$, we obtain (5.21). This completes the proof.

Proof of Proposition 5.10. We argue exactly the same as in the proof of Proposition 5.7 until (5.16) giving

$$
\operatorname{det}\left(I+P_{n}\left(\mathrm{e}^{A_{0}^{(n)}} \mathrm{e}^{A_{1}^{(n)}} \cdots \mathrm{e}^{\left.A_{N+1}^{(n)}-I\right) P_{n}}\right)\right.
$$

where

$$
A_{m}^{(n)}=\left(t_{m+1}^{(n)}-t_{m}^{(n)}\right) T\left(a\left(t_{m}^{(n)}\right)\right), \quad m=1, \ldots, N
$$

and

$$
A_{0}^{(n)}=-\sum_{m=1}^{N}\left(t_{m+1}^{(n)}-t_{m}^{(n)}\right) T\left(a_{+}\left(t_{m}^{(n)}\right)\right)
$$

and

$$
A_{N+1}^{(n)}=-\sum_{m=1}^{N}\left(t_{m+1}^{(n)}-t_{m}^{(n)}\right) T\left(a_{-}\left(t_{m}^{(n)}\right)\right)
$$

Hence it is clear that $\left.A^{(n}\right)_{0}+\cdots+A_{N+1}^{(n)}=0$. Moreover, each $A_{m}^{(n)}$ is a banded Toeplitz matrix with bandwidth $\max (p, q)$. Finally, we check the condition on the 
norm of the matrices. To this end,

$$
\begin{aligned}
\sum_{m=1}^{N}\left\|A_{m}^{(n)}\right\|_{\infty} & =\sum_{m=1}^{N}\left(t_{m+1}^{(n)}-t_{m}^{(n)}\right)\left\|T\left(a\left(t_{m}^{(n)}\right)\right)\right\|_{\infty} \\
& =\sum_{m=1}^{N}\left(t_{m+1}^{(n)}-t_{m}^{(n)}\right)\left\|a\left(t_{m}^{(n)}\right)\right\|_{\infty} \\
& \leq(\beta-\alpha) \sup _{t}\|a(t)\|_{\infty} \\
& \leq(\beta-\alpha) \sum_{j=-q}^{p} \sup _{t}\left|a_{j}(t)\right|
\end{aligned}
$$

The latter is finite, since we assume that the $a_{j}$ are piecewise continuous. A similar argument shows that also $\left\|A_{0}^{(n)}\right\|_{\infty},\left\|A_{N+1}^{(n)}\right\| \leq \sum_{j=-q}^{p} \sup _{t}\left|a_{j}(t)\right|$. Hence the last condition of Lemma 5.12 is also satisfied. It then follows from that lemma that

$$
\begin{gathered}
\lim _{n \rightarrow \infty} \operatorname{det}\left(I+P_{n}\left(\mathrm{e}^{A_{0}^{(n)}} \mathrm{e}^{A_{1}^{(n)}} \cdots \mathrm{e}^{A_{N+1}^{(n)}}-I\right) P_{n}\right) \\
=\lim _{n \rightarrow \infty} \operatorname{det}\left(\mathrm{e}^{A_{0}^{(n)}} \mathrm{e}^{A_{1}^{(n)}} \cdots \mathrm{e}^{A_{N+1}^{(n)}}\right) .
\end{gathered}
$$

The right-hand side is the exponential of a trace that we can compute in the same way as we have done in the proof of Proposition 5.7, which gives

$$
\begin{aligned}
\lim _{n \rightarrow \infty} \operatorname{det}\left(I+P_{n}\left(\mathrm{e}^{A_{0}^{(n)}} \mathrm{e}^{A_{1}^{(n)}} \cdots \mathrm{e}^{A_{N+1}^{(n)}}-I\right) P_{n}\right) \\
=\lim _{n \rightarrow \infty} \exp \left(2 \sum_{m_{1}=1}^{N_{n}}\left(t_{m_{1}+1}^{(n)}-t_{m_{1}}^{(n)}\right)\right. \\
\quad \times \sum_{m_{2}=m_{1}+1}^{N_{n}}\left(t_{m_{2}+1}^{(n)}-t_{m_{2}}^{(n)}\right) \sum_{\ell=1}^{\infty} \ell a_{\ell}\left(t_{m_{1}}^{(n)}\right) a_{-\ell}\left(t_{m \prime \prime}^{(n)}\right) \\
\left.\quad+\sum_{m=1}^{N_{n}}\left(t_{m+1}^{(n)}-t_{m}^{(n)}\right)^{2} \sum_{\ell=1}^{\infty} \ell a_{\ell}(m) a_{-\ell}(m)\right) .
\end{aligned}
$$

In the limit $n \rightarrow \infty$, the double sum in the exponent converges to a RiemannStieltjes integral and the single sum tends to zero. We thus proved the statement.

\section{Proofs of the main results.}

6.1. Proof of Theorem 2.2. To prove that the difference of the moments converges to zero, it is sufficient to prove that for $k \geq 2$ we have

$$
\mathcal{C}_{k}\left(X_{n}(f)\right)-\tilde{\mathcal{C}}_{k}\left(X_{n}(f)\right) \rightarrow 0,
$$


as $n \rightarrow \infty$, where the $\mathcal{C}_{k}$ 's are the cumulants defined in (4.7). By Lemmas 4.1 and 4.2 , and this means that we need to prove that, for given $k \geq 2$,

$$
C_{k}^{(n)}\left(f\left(1, \mathbb{J}_{1, S}\right), \ldots, f\left(N, \mathbb{J}_{N, S}\right)\right)-C_{k}^{(n)}\left(f\left(1, \tilde{\mathbb{J}}_{1, S}\right), \ldots, f\left(N, \tilde{\mathbb{J}}_{N, S}\right)\right) \rightarrow 0,
$$

as $n \rightarrow \infty$ for $S$ sufficiently large. The key ingredient is Corollary 5.6.

Since $x \mapsto f(m, x)$ is a polynomial and $\mathbb{J}_{m}$ and $\tilde{\mathbb{J}}_{m}$ are banded, it is not hard to see that (2.13) implies that, for given $k, \ell \in \mathbb{Z}$,

$$
\left(f\left(m, \mathbb{J}_{m}\right)\right)_{n+k, n+\ell}-\left(f\left(m, \tilde{\mathbb{J}}_{m}\right)\right)_{n+k, n+\ell} \rightarrow 0,
$$

as $n \rightarrow \infty$.

Now, let $k \in \mathbb{N}$. Note that for any $S \in \mathbb{N}$ we have that both $f\left(m, \mathbb{J}_{m, S}\right)$ and $f\left(m, \tilde{\mathbb{J}}_{m, S}\right)$ are banded matrices with a bandwidth $\rho$ that is independent of $n$. With $R_{n, 2 \rho(k+1)}$ as in Corollary 5.6, we then have

$$
\left\|R_{n, 2 \rho(k+1)}\left(f\left(m, \mathbb{J}_{m, S}\right)-f\left(m, \tilde{\mathbb{J}}_{m, S}\right)\right) R_{n, 2 \rho(k+1)}\right\|_{\infty} \rightarrow 0,
$$

as $n \rightarrow \infty$ for sufficiently large $S$. We also have by the first condition in Theorem 2.2 that there exists an $M>0$ such that

$$
\left\|R_{n, 2 \rho(k+1)} f\left(m, \tilde{\mathbb{J}}_{m, S}\right) R_{n, 2 \rho(k+1)}\right\|_{\infty}<M,
$$

for $n \in \mathbb{N}$. Hence, by (6.2), we can also choose $M$ to be large enough so that we have

$$
\left\|R_{n, 2 \rho(k+1)} f\left(m, \mathbb{J}_{m, S}\right) R_{n, 2 \rho(k+1)}\right\|_{\infty}<M,
$$

for $n \in \mathbb{N}$. The statement now follows by (6.1) and inserting (6.2), (6.3) and (6.4) for sufficiently large $S$ into the conclusion of Corollary 5.6.

6.2. Proof of Theorem 2.3. To prove that $X_{n}(f)-\mathbb{E} X_{n}(f)$ converges to a normally distributed random variable, it is sufficient to prove that the $k$ th cumulant converges to zero if $k \geq 3$ and to the stated value of the variance if $k=2$. By (5.3), this means that we need to show that, for given $k>2$,

$$
\lim _{n \rightarrow \infty} C_{k}^{(n)}\left(f\left(1, \mathbb{J}_{1, S}\right), \ldots, f\left(N, \mathbb{J}_{N, S}\right)\right)=0,
$$

for some sufficiently large $S$, and that the variance $C_{k}^{(n)}$ converges to the stated value.

Let $k \in \mathbb{N}$. The assumptions of the theorem imply that

$$
\lim _{n \rightarrow \infty}\left(\left(\mathbb{J}_{m}\right)_{n+k, n+l}-(T(a(m)))_{n+k, n+l}\right)=0,
$$

with $a(z)=\sum_{j=-q}^{p} a_{j}^{(m)} z^{j}$ where $a_{j}$ are the values in (2.15). Since $x \mapsto f(x, m)$ is a polynomial, it is not hard to see that also

$$
\lim _{n \rightarrow \infty}\left(\left(f\left(m, \mathbb{J}_{m, S}\right)\right)_{n+k, n+l}-(T(b(m)))_{k, l}\right)=0,
$$

for a sufficiently large $S$ and with $b(m)=\sum_{k} f_{k}^{(m)} z^{k}$ and $f_{k}^{(m)}$ as in (2.17). The statement then follows after applying Corollaries 5.6 and 5.8. 
6.3. Proof of Theorem 2.5. We now show how the results can be extended to allow for more general functions in the case (2.10). We recall that the variance of the linear statistics is given in terms of the kernel $K_{n, N}$ as in (4.1). Note that for fixed $m$ the kernel in this situation reads

$$
K_{n, N}(m, x, m, y)=\sum_{j=1}^{n} p_{j, m_{1}}(x) p_{j, m_{1}}(y)
$$

and hence $K_{n, N}(m, x, m, y)=K_{n, N}(m, y, m, x)$. This symmetry is a key property in the coming analysis. We start with the following estimate on the variance.

LEMMA 6.1. Let $f(m, x)$ be such that $\frac{\partial f}{\partial x}(m, x)$ is a bounded function. Then

$$
\operatorname{Var} \sum_{m=1}^{N} \sum_{j=1}^{n} f\left(m, x_{j}\left(t_{m}\right)\right) \leq \frac{N}{2} \sum_{m=1}^{N}\left\|\frac{\partial f}{\partial x}(m, x)\right\|_{\infty}^{2}\left\|\left[P_{n}, J_{m}\right]\right\|_{2}^{2} .
$$

Proof. Let us first deal with the special case that $N=1$. In that case, the variance of a linear statistic can be written as [with $K_{n, N}(x, 1, y, 1)$ shortened to $\left.K_{n, N}(x, y)\right]$

$$
\begin{aligned}
\operatorname{Var} \sum_{j=1}^{n} f\left(x_{j}(t)\right)= & \int f(x)^{2} K_{n, N}(x, x) \mathrm{d} \mu(x) \\
& -\iint f(x) f(y) K_{n, N}(x, y) K_{n, N}(y, x) \mathrm{d} \mu(x) \mathrm{d} \mu(y) .
\end{aligned}
$$

By using the fact that, by orthogonality, we have $\int K_{n, N}(x, y) K_{n, N}(y, x) \mathrm{d} \mu(y)=$ $K_{n, N}(x, x)$ we can rewrite this in symmetric form

$$
\begin{aligned}
& \operatorname{Var} \sum_{j=1}^{n} f\left(x_{j}(t)\right) \\
& \quad=\frac{1}{2} \iint(f(x)-f(y))^{2} K_{n, N}(x, y) K_{n, N}(y, x) \mathrm{d} \mu(x) \mathrm{d} \mu(y) .
\end{aligned}
$$

We now use the fact that $K_{n, N}(x, y)$ is symmetric [and hence $K_{n, N}(x, y) K_{n, N}(y$, $x) \geq 0$ ] to estimate this as

$$
\begin{aligned}
& \operatorname{Var} \sum_{j=1}^{n} f\left(x_{j}(t)\right) \\
& \quad \leq \frac{1}{2}\left\|f^{\prime}\right\|_{\infty} \iint(x-y)^{2} K_{n, N}(x, y) K_{n, N}(y, x) \mathrm{d} \mu(x) \mathrm{d} \mu(y) .
\end{aligned}
$$

It remains to estimate the double integral. To this end, we note that

$$
\frac{1}{2} \iint(x-y)^{2} K_{n, N}(x, y) K_{n, N}(y, x) \mathrm{d} \mu(x) \mathrm{d} \mu(y)=\left\|\left[P_{n}, J\right]\right\|_{2}^{2},
$$


proving the statement for $N=1$. For general $N$, we use the inequality $\operatorname{Var} \sum_{m=1}^{N} X_{m} \leq N \sum_{m=1}^{N} \operatorname{Var} X_{m}$, valid for any sum of random variables.

In the next lemma, we show that we can approximate the variance for linear statistics by linear statistic corresponding to polynomials.

LEMMA 6.2. Assume there exists a compact set $E \subset \mathbb{R}$ such that

(1) $S\left(\mu_{m}^{(n)}\right) \subset E$ for $m=1, \ldots, N$ and $n \in \mathbb{N}$,

or, more generally,

(2) for $k \in \mathbb{N}$ and $m=1, \ldots, N$ we have

$$
\int_{\mathbb{R} \backslash E}|x|^{k} K_{n, N}(m, x, m, x) \mathrm{d} \mu_{m}^{(n)}(x)=o(1 / n),
$$

as $n \rightarrow \infty$.

Then for any function $f$ such that $x \mapsto f(m, x)$ is a continuously differentiable function with at most polynomial growth at $\pm \infty$, we have

$$
\operatorname{Var} X_{n}(f) \leq \frac{N}{2} \sum_{m=1}^{N} \sup _{x \in E}\left|\frac{\partial f}{\partial x}(m, x)\right|^{2}\left\|\left[P_{n}, J_{m}\right]\right\|_{2}^{2}+o(1),
$$

as $n \rightarrow \infty$.

PROOF. If the supports of $\mu^{(m)}$ all lie within a compact set of $\mathbb{R}$ (independent of $n$ ), then this is simply Lemma 6.1. Now suppose the measure is not compactly supported but condition (2) is satisfied with some $E$. Then we write again

$$
\operatorname{Var} \sum_{m=1}^{N} \sum_{j=1}^{n} f\left(m, x_{j}\left(t_{m}\right)\right) \leq N \sum_{m=1}^{N} \operatorname{Var} \sum_{j=1}^{n} f\left(m, x_{j}\left(t_{m}\right)\right) .
$$

Each term in the sum at the right-hand side can be written in the form of (6.5) and we have

$$
\begin{array}{rl}
\operatorname{Var} \sum_{j=1}^{n} & f\left(m, x_{j}\left(t_{m}\right)\right) \\
= & \frac{1}{2} \iint_{E \times E}(f(m, x)-f(m, y))^{2} \\
& \times K_{n, N}(m, x, m, y) K_{n, N}(m, y, m, x) \mathrm{d} \mu(x) \mathrm{d} \mu(y) \\
& +\frac{1}{2} \iint_{(\mathbb{R} \backslash E) \times E}(f(m, x)-f(m, y))^{2} \\
& \times K_{n, N}(m, x, m, y) K_{n, N}(m, y, m, x) \mathrm{d} \mu(x) \mathrm{d} \mu(y)
\end{array}
$$




$$
\begin{aligned}
& +\frac{1}{2} \iint_{\mathbb{R} \times(\mathbb{R} \backslash E)}(f(m, x)-f(m, y))^{2} \\
& \times K_{n, N}(m, x, m, y) K_{n, N}(m, y, m, x) \mathrm{d} \mu(x) \mathrm{d} \mu(y) .
\end{aligned}
$$

Now, since

$$
\begin{aligned}
K_{n, N}(m, x, m, y) K_{n, N}(m, y, m, x) & \leq K_{n, N}(m, x, m, x) K_{n, N}(m, y, m, y), \\
\int_{\mathbb{R}} K_{n, N}(m, y, m, y) \mathrm{d} \mu(y) & =n,
\end{aligned}
$$

and $g$ has at most polynomial growth at infinity, we have

$$
\begin{aligned}
& \iint_{(\mathbb{R} \backslash E) \times E}(f(m, x)-f(m, y))^{2} \\
& \times K_{n, N}(m, x, m, y) K_{n, N}(m, y, m, x) \mathrm{d} \mu(x) \mathrm{d} \mu(y) \\
& \leq \iint_{(\mathbb{R} \backslash E) \times E}(f(m, x)-f(m, y))^{2} \\
& \quad \times K_{n, N}(m, x, m, x) K_{n, N}(m, y, m, y) \mathrm{d} \mu(x) \mathrm{d} \mu(y) \\
& =o(1 / n) \int K_{n, N}(m, y, m, y) \mathrm{d} \mu(y)=o(1),
\end{aligned}
$$

as $n \rightarrow \infty$. For the same reasons,

$$
\begin{aligned}
& \iint_{\mathbb{R} \times(\mathbb{R} \backslash E)}(f(m, x)-f(m, y))^{2} \\
& \quad \times K_{n, N}(m, x, m, y) K_{n, N}(m, y, m, x) \mathrm{d} \mu(x) \mathrm{d} \mu(y)=o(1),
\end{aligned}
$$

as $n \rightarrow \infty$. Moreover,

$$
\begin{aligned}
\iint_{E \times E} & (f(m, x)-f(m, y))^{2} \\
\times & K_{n, N}(m, x, m, y) K_{n, N}(m, y, m, x) \mathrm{d} \mu(x) \mathrm{d} \mu(y) \\
\leq & \sup _{x \in E}\left|\frac{\partial f}{\partial x}(m, x)\right|^{2} \iint_{E \times E}(x-y)^{2} \\
& \times K_{n, N}(m, x, m, y) K_{n, N}(m, y, m, x) \mathrm{d} \mu(x) \mathrm{d} \mu(y) \\
\leq & \sup _{x \in E}\left|\frac{\partial f}{\partial x}(m, x)\right|^{2} \iint_{\mathbb{R} \times \mathbb{R}}(x-y)^{2} \\
& \times K_{n, N}(m, x, m, y) K_{n, N}(m, y, m, x) \mathrm{d} \mu(x) \mathrm{d} \mu(y) \\
= & \sup _{x \in E}\left|\frac{\partial f}{\partial x}(m, x)\right|^{2}\left\|\left[P_{n}, J_{m}\right]\right\|_{2}^{2} .
\end{aligned}
$$


By inserting (6.10), (6.11) and (6.12) into (6.9) and the result into (6.8), we obtain the statement.

We need one more lemma.

Lemma 6.3. Let $a_{0}^{(m)} \in \mathbb{R}$ and $a_{1}^{(m)}>0$ for $m=1, \ldots, N$. Then for any real valued function $f$ on $\{1, \ldots, N\} \times \mathbb{R}$ such that $x \mapsto f(m, x)$ is continuously differentiable, we have that

$$
\sigma(f)^{2}=\sum_{k=1}^{\infty} \sum_{m_{1}, m_{2}=1}^{\infty} \mathrm{e}^{-\left|\tau_{m_{1}}-\tau_{m_{2}}\right| k} f_{k}^{\left(m_{1}\right)} f_{k}^{\left(m_{2}\right)},
$$

is finite, where

$$
f_{k}^{(m)}=\frac{1}{2 \pi} \int_{0}^{2 \pi} f\left(m, a_{0}^{(m)}+2 a_{1}^{(m)} \cos \theta\right) \mathrm{e}^{-\mathrm{i} k \theta} \mathrm{d} \theta .
$$

Moreover, for some constant $C$ we have

$$
\sigma(f)^{2} \leq C N \sum_{m=1}^{N} \sup _{x \in F_{m}}\left|\frac{\partial f}{\partial x}(m, x)\right|^{2},
$$

where $F_{m}=\left[a_{0}^{(m)}-2 a_{1}^{(m)}, a_{0}^{(m)}+2 a_{1}^{(m)}\right]$.

PROOF. We start by recalling Remark 2.4 on the symmetric case. By the same arguments leading to (2.23), we see that we can write

$$
\sigma(f)^{2}=\sum_{k=1}^{\infty} \int_{-\infty}^{\infty}\left|\sum_{m=1}^{N} \mathrm{e}^{-\mathrm{i} \tau_{m} \omega} k f_{k}^{(m)}\right|^{2} \frac{\mathrm{d} \omega}{k^{2}+\omega^{2}}
$$

showing that $\sigma(f)^{2}$ is indeed positive (if finite). To show that it is finite, we note by applying Cauchy-Schwarz on the sum over $m$ and using

$$
\int \frac{\mathrm{d} \omega}{k^{2}+\omega^{2}} \leq \int \frac{\mathrm{d} \omega}{1+\omega^{2}}=\pi
$$

it easily follows that

$$
\sigma(f)^{2} \leq C_{0} N \sum_{m=1}^{N} \sum_{k=1}^{\infty} k^{2}\left|f_{k}^{(m)}\right|^{2}
$$

for some constant $C_{0}>0$. Then by integration by parts we find

$$
k f_{k}^{(m)}=\frac{1}{2 \pi} \int_{0}^{2 \pi} \frac{\partial f}{\partial x}\left(m, a_{0}^{(m)}+2 a_{1}^{(m)} \cos \theta\right) a_{1}^{(m)} \sin \theta \mathrm{e}^{-\mathrm{i} k \theta} \mathrm{d} \theta .
$$


By applying Parseval and using the compactness of $F$ to estimate the $\mathbb{L}_{2}$ norm by the $\mathbb{L}_{\infty}$ norm, we find there exists a constant $C$ such that

$$
\sum_{k=1}^{\infty} k^{2}\left|f_{k}^{(m)}\right|^{2} \leq C \sup _{x \in F_{m}}\left|\frac{\partial f}{\partial x}(m, x)\right|^{2} .
$$

By inserting this back into (6.14), we obtain the statement.

Proof of TheOrem 2.5. For any function $p$ on $\{1, \ldots, N\} \times \mathbb{R}$ such that $x \mapsto p(m, x)$ is a polynomial, we can write

$$
\begin{aligned}
& \left|\mathbb{E}\left[\mathrm{e}^{\mathrm{i} t X_{n}(f)}\right]-\mathrm{e}^{-\frac{\sigma(f)^{2} t^{2}}{2}}\right| \\
& \leq\left|\mathbb{E}\left[\mathrm{e}^{\mathrm{i} t X_{n}(f)}\right]-\mathbb{E}\left[\mathrm{e}^{\mathrm{i} t X_{n}(p)}\right]\right| \\
& \quad+\left|\mathbb{E}\left[\mathrm{e}^{\mathrm{i} t X_{n}(p)}\right]-\mathrm{e}^{-\frac{\sigma(p)^{2} t^{2}}{2}}\right|+\left|\mathrm{e}^{-\frac{\sigma(p)^{2} t^{2}}{2}}-\mathrm{e}^{-\frac{\sigma(f)^{2} t^{2}}{2}}\right| .
\end{aligned}
$$

We claim that we can choose $p$ such that the first and last term at the right-hand side are small. Let $G$ be a compact set containing both the compact set $E$ and all $F_{m}$ from Lemmas 6.2 and 6.3, respectively. For $\varepsilon>0$, choose $p$ such that

$$
\sum_{m=1}^{N} \sup _{x \in F_{m}}\left|\frac{\partial f}{\partial x}(m, x)-\frac{\partial p}{\partial x}(m, x)\right|^{2}<\varepsilon,
$$

and such that $p(m, x)$ is a polynomial in $x$. Also note that by the assumptions of Theorem 2.3, there exists a constant $C_{0}$ such that

$$
\left\|\left[P_{n}, J_{m}\right]\right\|_{2} \leq C_{0},
$$

for $n \in \mathbb{N}$ and $m=1, \ldots, N$.

We now recall that for any two real valued random variables $X, Y$ with $\mathbb{E} X=$ $\mathbb{E} Y$ we have the identity

$$
\begin{aligned}
\left|\mathbb{E}\left[\mathrm{e}^{\mathrm{i} t X}\right]-\mathbb{E}\left[\mathrm{e}^{\mathrm{i} t Y}\right]\right| & =\left|\mathbb{E}\left[\mathrm{e}^{\mathrm{i} t X}-\mathrm{e}^{\mathrm{i} t Y}\right]\right| \\
& \leq \mathbb{E}\left[\left|1-\mathrm{e}^{\mathrm{i} t(X-Y)}\right|\right] \\
& \leq|t| \mathbb{E}[|X-Y|] \\
& \leq|t| \sqrt{\operatorname{Var}(X-Y)}
\end{aligned}
$$

for $t \in \mathbb{R}$. This means by Lemma 6.2, (6.16) and (6.17) that

$$
\left|\mathbb{E}\left[\mathrm{e}^{\mathrm{i} t X_{n}(f)}\right]-\mathbb{E}\left[\mathrm{e}^{\mathrm{i} t X_{n}(p)}\right]\right| \leq|t| \sqrt{\operatorname{Var} X_{n}(f-p)} \leq|t| C_{1} \varepsilon,
$$

for $t \in \mathbb{R}$ some constant $C_{1}$. Moreover, by Lemma 6.3 and (6.16) we have that

$$
\left|\mathrm{e}^{-\frac{\sigma(p)^{2} t^{2}}{2}}-\mathrm{e}^{-\frac{\sigma(f)^{2} t^{2}}{2}}\right| \leq C_{2} \varepsilon
$$


for some constant $C_{2}$. By substituting this back into (6.15) and applying Theorem 2.3, we obtain

$$
\begin{aligned}
\left|\mathbb{E}\left[\mathrm{e}^{\mathrm{i} t X_{n}(f)}\right]-\mathrm{e}^{-\frac{\sigma(f)^{2} t^{2}}{2}}\right| & \leq\left(C_{1}+C_{2}\right) \varepsilon\left|\mathbb{E}\left[\mathrm{e}^{\mathrm{i} t X_{n}(p)}\right]-\mathrm{e}^{-\frac{\sigma(p)^{2} t^{2}}{2}}\right| \\
& \rightarrow\left(C_{1}+C_{2}\right) \varepsilon
\end{aligned}
$$

as $n \rightarrow \infty$. Since $\varepsilon$ was arbitrary, we have $\left|\mathbb{E}\left[\mathrm{e}^{\mathrm{i} t X_{n}(f)}\right]-\mathrm{e}^{-\frac{\sigma(f)^{2} t^{2}}{2}}\right| \rightarrow 0$ as $n \rightarrow \infty$ and the statement follows.

6.4. Proof of Theorem 2.6. The proof is a simple extension of the proof of Theorem 2.2 and is again a consequence of Corollary 5.6. The main difference being that (6.2), (6.3) and (6.4) are not sufficient, since the the number of terms in Corollary 5.6 grows with $n$. Instead, we replace (6.2) with

$$
\begin{aligned}
\sum_{m=1}^{N_{n}} \| & R_{n, 2 \rho(k+1)}\left(f\left(m, \mathbb{J}_{m, S}\right)-f\left(m, \tilde{\mathbb{J}}_{m, S}\right)\right) R_{n, 2 \rho(k+1)} \|_{\infty} \\
\leq & \sum_{m=1}^{N_{n}}\left(t_{m+1}^{(n)}-t_{m}^{(n)}\right) \\
& \times\left\|R_{n, 2 \rho(k+1)}\left(g\left(m, \mathbb{J}_{m, S}\right)-g\left(m, \tilde{\mathbb{J}}_{m, S}\right)\right) R_{n, 2 \rho(k+1)}\right\|_{\infty} \\
\leq & (\beta-\alpha) \\
& \times \sup _{m=1, \ldots, N_{n}}\left\|R_{n, 2 \rho(k+1)}\left(g\left(m, \mathbb{J}_{m, S}\right)-g\left(m, \tilde{\mathbb{J}}_{m, S}\right)\right) R_{n, 2 \rho(k+1)}\right\|_{\infty} \\
\rightarrow & 0,
\end{aligned}
$$

as $n \rightarrow \infty$ for sufficiently large $S$, by the condition (2.25) (and the exact same reasoning as in the beginning of the proof of Theorem 2.5). We also replace (6.3) by

$$
\begin{aligned}
& \sum_{m=1}^{N_{n}}\left\|R_{n, 2 \rho(k+1)} f\left(m, \tilde{\mathbb{J}}_{m, S}\right) R_{n, 2 \rho(k+1)}\right\|_{\infty} \\
& \quad \leq \sum_{m=1}^{N_{n}}\left(t_{m+1}^{(n)}-t_{m}^{(n)}\right)\left\|R_{n, 2 \rho(k+1)} g\left(m, \tilde{\mathbb{J}}_{m, S}\right) R_{n, 2 \rho(k+1)}\right\|_{\infty} \\
& \quad \leq(\beta-\alpha) \sup _{m=1, \ldots, N_{n}}\left\|R_{n, 2 \rho(k+1)} g\left(m, \tilde{\mathbb{J}}_{m, S}\right) R_{n, 2 \rho(k+1)}\right\|_{\infty},
\end{aligned}
$$

which is bounded in $n$ by the assumption in the theorem and the fact that $g(m, x)$ is a polynomial in $x$. This also shows that

$$
\sum_{m=1}^{N_{n}}\left\|R_{n, 2 \rho(k+1)} f\left(m, \mathbb{J}_{m, S}\right) R_{n, 2 \rho(k+1)}\right\|_{\infty},
$$


is also bounded $n$. Hence, by inserting these identities in the conclusion of Corollary 5.6 we proved the statement.

6.5. Proof of Theorem 2.7. The proof is exactly the same as the proof of Theorem 2.3, with the only difference that we now rely on (the proof of) Theorem 2.6 and use Corollary 5.11 in the final step instead of Corollary 5.8.

6.6. Proof of Theorem 2.9. The proof of Theorem 2.9 also follows the same arguments. We start with the following adjustment of Lemma 6.2.

LEMMA 6.4. Assume there exists a compact set $E \subset \mathbb{R}$ such that

(1) $S\left(\mu_{m}^{(n)}\right) \subset E$ for $m=1, \ldots, N_{n}$ and $n \in \mathbb{N}$,

or, more generally,

(2) for $k \in \mathbb{N}$, we have

$$
\sup _{m=1, \ldots, N_{n}} \int_{\mathbb{R} \backslash E}|x|^{k} K_{n, N}(m, x, m, x) \mathrm{d} \mu_{m}^{(n)}(x)=o(1 / n),
$$

as $n \rightarrow \infty$.

Then for any function $g$ such that $x \mapsto g(t, x)$ is a continuously differentiable function with at most polynomial growth at $\pm \infty$, that is, $g(t, x)=\mathcal{O}\left(|x|^{M}\right)$ as $|x| \rightarrow \infty$, for some $M$ independent of $t \in I$, we have

$$
\begin{aligned}
\operatorname{Var} X_{n}(f) \leq & \frac{N}{2} \sum_{m=1}^{N}\left(t_{m+1}^{(n)}-t_{m}^{(n)}\right)^{2}\left\|\frac{\partial g}{\partial x}\right\|_{\mathbb{L}_{\infty}(I \times E)}^{2} \\
& \times \sup _{m=1, \ldots, N_{n}}\left\|\left[P_{n}, J_{m}\right]\right\|_{2}^{2}+o(1),
\end{aligned}
$$

as $n \rightarrow \infty$.

PROOF. We use the same arguments as in the proof of Lemma 6.2. The only possible issue that we need to address is that in (6.7) the constant in the $o(1)$ term my depend on $N$ and hence, in the present situation, also on $n$. However, by the fact we take the supremum over $m$ in (6.21) we have that the constant in the $o(1)$ terms in (6.10) and (6.11) can be chosen such that they do not on $n$. By following the same proof, we see that the constant in the $o(1)$ does not depend on $n$, and hence we have

$$
\operatorname{Var} X_{n}(f) \leq \frac{N_{n}}{2} \sum_{m=1}^{N_{n}} \sup _{x \in E}\left|\frac{\partial f}{\partial x}(m, x)\right|^{2}\left\|\left[P_{n}, J_{m}\right]\right\|_{2}^{2}+o(1),
$$

as $n \rightarrow \infty$. Since $f$ is considered to be of the special type (2.24), we rewrite the 
latter in terms of $g$,

$$
\operatorname{Var} X_{n}(f) \leq \frac{N_{n}}{2} \sum_{m=1}^{N_{n}}\left(t_{m+1}^{(n)}-t_{m}^{(n)}\right)^{2}\left\|\frac{\partial g}{\partial x}\right\|_{\mathbb{L}_{\infty}(I \times E)}^{2}\left\|\left[P_{n}, J_{m}\right]\right\|_{2}^{2}+o(1),
$$

as $n \rightarrow \infty$. The statement now follows by estimating the summands in terms of their suprema.

LEMMA 6.5. Let $a_{0}^{(m)}$ and $a_{1}^{(m)}$ be piecewise continuous function on I for $m=1, \ldots, N_{n}$. Then for any real valued function $g$ on $\{1, \ldots, N\} \times \mathbb{R}$ such that $x \mapsto g(\tau, x)$ is continuously differentiable, we have that

$$
\sigma(g)^{2}=\sum_{k=1}^{\infty} \iint_{I \times I} \mathrm{e}^{-\left|\tau_{1}(t)-\tau_{2}(t)\right| k} g_{k}\left(t_{1}\right) g_{k}\left(t_{2}\right) \mathrm{d} t_{1} \mathrm{~d} t_{2},
$$

is finite, where

$$
g_{k}(t)=\frac{1}{2 \pi} \int_{0}^{2 \pi} g\left(m, a_{0}(t)+2 a_{1}(t) \cos t\right) \mathrm{e}^{-\mathrm{i} k \theta} \mathrm{d} \theta .
$$

Moreover, for some constant $C$ we have

$$
\sigma(f)^{2} \leq C\left\|\frac{\partial g}{\partial x}\right\|_{\mathbb{L}_{\infty}(F)}^{2}
$$

where $F=\left\{(t, x) \mid x \in\left[a_{0}(t)-2 a_{1}(t), a_{0}(t)+2 a_{1}(t)\right], t \in I\right\}$.

PROOF. As in (2.28) we rewrite $\sigma(g)^{2}$ as

$$
\sigma(g)^{2}=\frac{1}{\pi} \sum_{k=1}^{\infty} \int \frac{k^{2}}{\omega^{2}+k^{2}}\left|\int_{I} \mathrm{e}^{-\mathrm{i} \omega \tau(t)} g_{k}(t) \mathrm{d} t\right|^{2} \mathrm{~d} \omega,
$$

showing that $\sigma(g)^{2}$ is positive (if finite). By applying the Cauchy-Schwarz inequality to the integral over $t$, the estimate (6.13) and the Plancherel Theorem we have

$$
\sigma(g)^{2} \leq(\beta-\alpha) \pi \sum_{k=1}^{\infty} \int_{\mathbb{R}} k^{2}\left|g_{k}(t)\right|^{2} \mathrm{~d} t
$$

Then by integration by parts we find

$$
k g f_{k}(t)=\frac{1}{2 \pi} \int_{0}^{2 \pi} \frac{\partial g}{\partial x}\left(t, a_{0}(t)+2 a_{1}(t) \cos \theta\right) a_{1}(t) \sin \theta \mathrm{e}^{-\mathrm{i} k \theta} \mathrm{d} \theta .
$$

By applying Parseval and estimating the $\mathbb{L}_{2}$ norm by the $\mathbb{L}_{\infty}$ norm, we find there 
exists a constant $C$ such that

$$
\sum_{k=1}^{\infty} k^{2}\left|g_{k}(t)\right|^{2} \leq C\left\|\frac{\partial g}{\partial x}\right\|_{\mathbb{L}_{\infty}(F)}^{2},
$$

and this completes the proof.

PROOF OF THEOREM 2.9. The proof of is now identical to the proof of Theorem 2.9, with only difference being that we use Theorem 2.7, Lemmas 6.4 and 6.5 instead of Theorem 2.3 and Lemmas 6.2 and 6.3.

\section{REFERENCES}

[1] Akemann, G., Baik, J. and Di Francesco, P., eds. (2011). The Oxford Handbook of Random Matrix Theory. Oxford Univ. Press, Oxford. MR2920518

[2] Anderson, G. W., Guionnet, A. and Zeitouni, O. (2010). An Introduction to Random Matrices. Cambridge Studies in Advanced Mathematics 118. Cambridge Univ. Press, Cambridge. MR2760897

[3] Bleher, P. M. and KuiJlaars, A. B. J. (2005). Integral representations for multiple Hermite and multiple Laguerre polynomials. Ann. Inst. Fourier (Grenoble) 55 2001-2014. MR2187942

[4] Borodin, A. (1999). Biorthogonal ensembles. Nuclear Phys. B 536 704-732. MR1663328

[5] Borodin, A. (2011). Determinantal point processes. In The Oxford Handbook of Random Matrix Theory 231-249. Oxford Univ. Press, Oxford. MR2932631

[6] Borodin, A. (2014). CLT for spectra of submatrices of Wigner random matrices, II: Stochastic evolution. In Random Matrix Theory, Interacting Particle Systems, and Integrable Systems. Math. Sci. Res. Inst. Publ. 65 57-69. Cambridge Univ. Press, New York. MR3380682

[7] Borodin, A. and Bufetov, A. (2014). Plancherel representations of $U(\infty)$ and correlated Gaussian free fields. Duke Math. J. 163 2109-2158. MR3263029

[8] Borodin, A. and Ferrari, P. L. (2014). Anisotropic growth of random surfaces in $2+1$ dimensions. Comm. Math. Phys. 325 603-684. MR3148098

[9] Borodin, A. and Gorin, V. (2015). General $\beta$-Jacobi corners process and the Gaussian free field. Comm. Pure Appl. Math. 68 1774-1844. MR3385342

[10] Borodin, A. and Olshanski, G. (2006). Markov processes on partitions. Probab. Theory Related Fields 135 84-152. MR2214152

[11] Böttcher, A. and Silbermann, B. (1999). Introduction to Large Truncated Toeplitz Matrices. Universitext. Springer, New York. MR1724795

[12] BREUER, J. and DUITS, M. (2016). Universality of mesoscopic fluctuations for orthogonal polynomial ensembles. Comm. Math. Phys. 342 491-531. MR3459158

[13] Breuer, J. and Duits, M. (2017). Central limit theorems for biorthogonal ensembles and asymptotics of recurrence coefficients. J. Amer. Math. Soc. 30 27-66. MR3556288

[14] Bufetov, A. and Gorin, V. Fluctuations of particle systems determined by Schur generating functions. ArXiv preprint. Available at arXiv:1604.01110.

[15] Costin, O. and Lebowitz, J. L. (1995). Gaussian fluctuation in random matrices. Phys. Rev. Lett. 75 69-72. MR3155254

[16] Daems, E. and KuiJlaArs, A. B. J. (2007). Multiple orthogonal polynomials of mixed type and non-intersecting Brownian motions. J. Approx. Theory 146 91-114. MR2327475 
[17] Deift, P., Kriecherbauer, T., Mclaughlin, K. T.-R., Venakides, S. and Zhou, X. (1999). Uniform asymptotics for polynomials orthogonal with respect to varying exponential weights and applications to universality questions in Random Matrix Theory. Comm. Pure Appl. Math. 52 1335-1425. MR1702716

[18] Doumerc, Y. (2005). Matrices aléatoires, processus stochastiques et groupes de réflexions Ph.D. thesis.

[19] Duits, M. (2013). Gaussian free field in an interlacing particle system with two jump rates. Comm. Pure Appl. Math. 66 600-643. MR3020314

[20] Duits, M., Geudens, D. and Kuijlaars, A. B. J. (2011). A vector equilibrium problem for the two-matrix model in the quartic/quadratic case. Nonlinearity 24 951-993. MR2772631

[21] Duits, M., KuiJlaars, A. B. J. and Mo, M. Y. (2012). The Hermitian two matrix model with an even quartic potential. Mem. Amer. Math. Soc. $217 \mathrm{v}+105$. MR2934329

[22] Dyson, F. J. (1962). A Brownian-motion model for the eigenvalues of a random matrix. J. Math. Phys. 3 1191-1198. MR0148397

[23] Ehrhardt, T. (2003). A generalization of Pincus' formula and Toeplitz operator determinants. Arch. Math. (Basel) 80 302-309. MR1981184

[24] Gohberg, I., Goldberg, S. and Krupnik, N. (2000). Traces and Determinants of Linear Operators. Operator Theory: Advances and Applications 116. Birkhäuser, Basel. MR1744872

[25] GORIN, V. E. (2008). Nonintersecting paths and the Hahn orthogonal polynomial ensemble. Funktsional. Anal. i Prilozhen. 42 23-44, 96. MR2454474

[26] Gorin, V. E. (2008). Noncolliding Jacobi diffusions as the limit of Markov chains on the Gelfand-Tsetlin graph. Zap. Nauchn. Sem. S.-Peterburg. Otdel. Mat. Inst. Steklov. (POMI) 360 91-123, 296. MR2759741

[27] Janson, S. (1997). Gaussian Hilbert Spaces. Cambridge Tracts in Mathematics 129. Cambridge Univ. Press, Cambridge. MR1474726

[28] Johansson, K. (1998). On fluctuations of eigenvalues of random Hermitian matrices. Duke Math. J. 91 151-204. MR1487983

[29] Johansson, K. (2005). The Arctic circle boundary and the Airy process. Ann. Probab. 33 1-30. MR2118857

[30] JoHAnsson, K. (2005). Non-intersecting, simple, symmetric random walks and the extended Hahn kernel. Ann. Inst. Fourier (Grenoble) 55 2129-2145. MR2187949

[31] Johansson, K. (2006). Random matrices and determinantal processes. In Mathematical Statistical Physics 1-55. Elsevier B. V., Amsterdam. MR2581882

[32] Kenyon, R. (2008). Height fluctuations in the honeycomb dimer model. Comm. Math. Phys. 281 675-709. MR2415464

[33] Koekoek, R., Lesky, P. A. and Swarttouw, R. F. (2010). Hypergeometric Orthogonal Polynomials and Their q-Analogues. Springer Monographs in Mathematics. Springer, Berlin. MR2656096

[34] KöNIG, W. (2005). Orthogonal polynomial ensembles in probability theory. Probab. Surv. 2 385-447. MR2203677

[35] König, W. and O'Connell, N. (2001). Eigenvalues of the Laguerre process as non-colliding squared Bessel processes. Electron. Commun. Probab. 6 107-114. MR1871699

[36] KöNig, W., O'Connell, N. and Roch, S. (2002). Non-colliding random walks, tandem queues, and discrete orthogonal polynomial ensembles. Electron. J. Probab. 7 no. 5, 24. MR1887625

[37] Kunn, J. (2014). The Gaussian free field in interlacing particle systems. Electron. J. Probab. 19 no. 72,31 . MR3256872 
[38] KuijlaArs, A. B. J. (2010). Multiple orthogonal polynomial ensembles. In Recent Trends in Orthogonal Polynomials and Approximation Theory. Contemp. Math. 507 155-176. Amer. Math. Soc., Providence, RI. MR2647568

[39] LyOns, R. (2003). Determinantal probability measures. Publ. Math. Inst. Hautes Études Sci. 98 167-212. MR2031202

[40] O'Connell, N. (2003). Conditioned random walks and the RSK correspondence. J. Phys. A 36 3049-3066. MR1986407

[41] O'CONNELL, N. and YOR, M. (2002). A representation for non-colliding random walks. Electron. Commun. Probab. 7 1-12. MR1887169

[42] OlshansKi, G. (2010). Laguerre and Meixner symmetric functions, and infinite-dimensional diffusion processes. Zap. Nauchn. Sem. S.-Peterburg. Otdel. Mat. Inst. Steklov. (POMI) 378 81-110, 230. MR2749298

[43] Petrov, L. (2015). Asymptotics of uniformly random lozenge tilings of polygons. Gaussian free field. Ann. Probab. 43 1-43. MR3298467

[44] Schoutens, W. (2000). Stochastic Processes and Orthogonal Polynomials. Lecture Notes in Statistics 146. Springer, New York. MR1761401

[45] ShefField, S. (2007). Gaussian free fields for mathematicians. Probab. Theory Related Fields 139 521-541. MR2322706

[46] Simon, B. (2005). Trace Ideals and Their Applications, 2nd ed. Mathematical Surveys and Monographs 120. Amer. Math. Soc., Providence, RI. MR2154153

[47] Simon, B. (2011). Szegós Theorem and Its Descendants: Spectral Theory for $L^{2}$ Perturbations of Orthogonal Polynomials. Princeton Univ. Press, Princeton, NJ. MR2743058

[48] Soshnikov, A. (2000). Determinantal random point fields. Uspekhi Mat. Nauk 55 107-160. MR1799012

[49] Soshnikov, A. (2002). Gaussian limit for determinantal random point fields. Ann. Probab. 30 171-187. MR1894104

[50] VAn Assche, W. and Coussement, E. (2001). Some classical multiple orthogonal polynomials. J. Comput. Appl. Math. 127 317-347. MR1808581

\author{
DEPARTMENT OF MATHEMATICS \\ ROYAL INSTITUTE OF TECHNOLOGY (KTH) \\ STOCKHOLM LINDSTEDSVÄGEN 25 \\ SE-10044 SWEDEN \\ E-MAIL: duits@kth.se
}

